\title{
Numerical Sequence Recognition: Is Familiarity or Ordinality the Primary Factor in Performance?
}

\author{
by
}

\author{
Angelle Bourassa
}

A thesis submitted to the Faculty of Graduate and Postdoctoral Affairs in partial fulfillment of the requirements for the degree of

\author{
Master of Arts \\ in
}

Psychology

Carleton University

Ottawa, Ontario

(C) 2014

Angelle Bourassa 


\begin{abstract}
Lyons and Beilock (2009) suggested that the degree of ordinal association in 3-digit numerical sequences is a primary factor in the speed and accuracy with which people recognize numerical sequences. Using two experiments I examined an alternative hypothesis, specifically that having automatic access to a larger set of memorized (i.e., familiar) sequences is the determining factor in performance. Participants were shown four types of ordered stimuli, with corresponding unordered sequences. In general, highly-skilled participants responded faster than their less-skilled counterparts. All participants were slower to reject unordered sequences that shared numbers with highly familiar sequences (e.g., 31 2) than with relatively unfamiliar unordered sequences (e.g., 712 ): this pattern is referred to as an interference effect. Participants were faster to identify familiar ascending than descending sequences, despite being equally ordered. These results support familiarity, and not ordinality, as the determining factor in sequence recognition.
\end{abstract}




\section{Acknowledgements}

I would like to take the time to thank first and foremost my thesis supervisor, Dr. JoAnne LeFevre. Her patience, advice, and assistance, have been invaluable to me and the skills I have learned from her will continue to serve me well in my future endeavors. I would also like to thank my thesis committee members in advance for their constructive feedback and insight on my research.

I would also like to thank Matt, Claire, Chunyun, Dean, Carolina, Kristina, Carla, Ozlem, Geoffrey, and all of my colleagues at the university, all of whom whom have immensely helped me with their amazing advice, feedback, and involvement in my research.

Finally, I would like to thank my family for supporting me physically and emotionally with my busy work schedule, my best friend Lucy for her always positive perspective and encouragement, and my wonderful boyfriend Charles, whose constant support, humor, and epic computer programming skills have helped me along every step of the way. 
Table of Contents

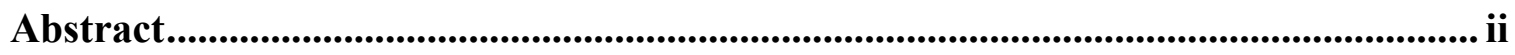

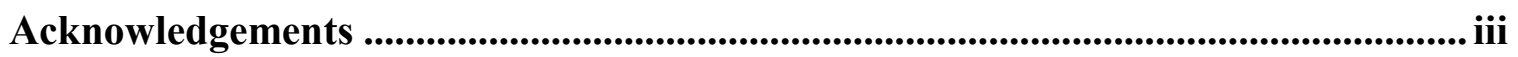

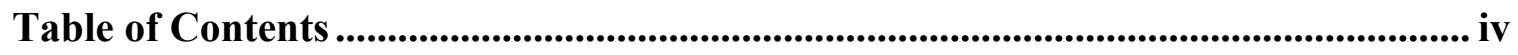

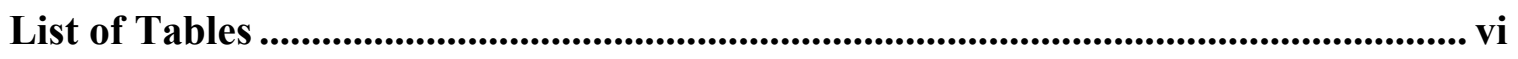

List of Illustrations.................................................................................................. vii

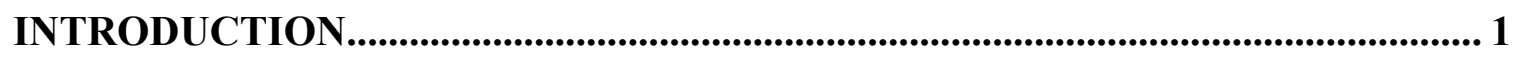

Numerical Cognition ............................................................................. 2

Familiarity and Arithmetic Performance ................................................. 3

Factors Influencing Numerical Sequence Recognition.................................. 5

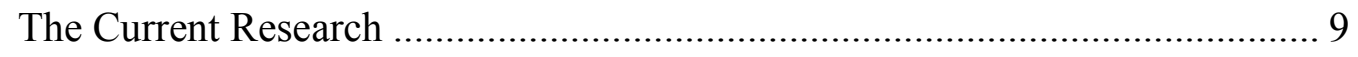

Experiment 1 ........................................................................................................................ 14

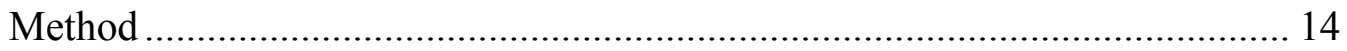

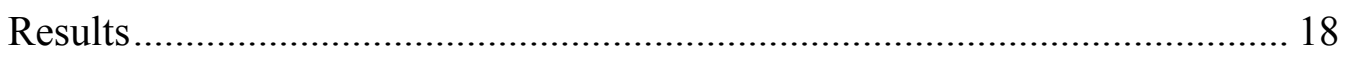

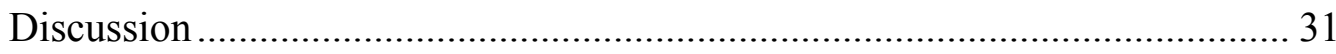

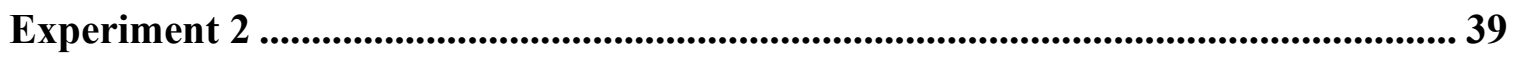

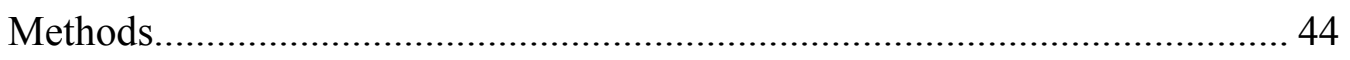

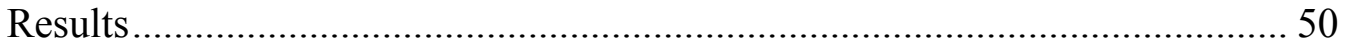

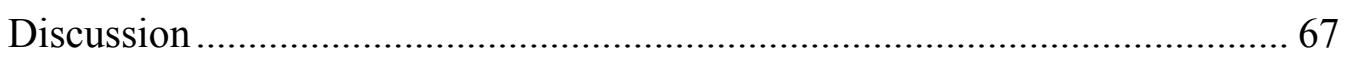

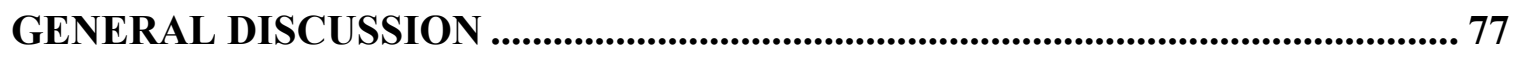

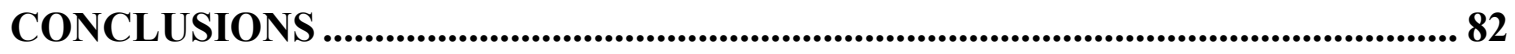

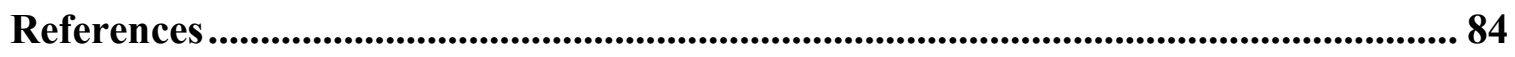

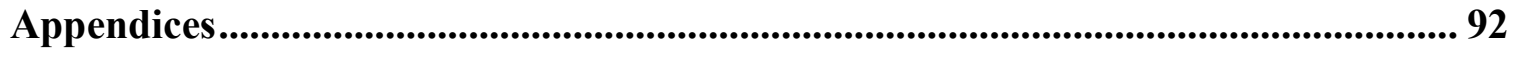




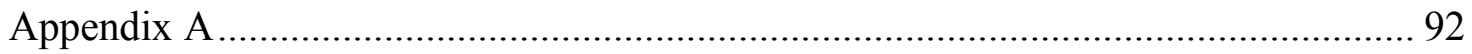

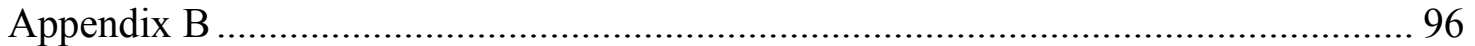




\section{List of Tables}

Table 1 Sample ordered and unordered sequences for each category in the ordering task

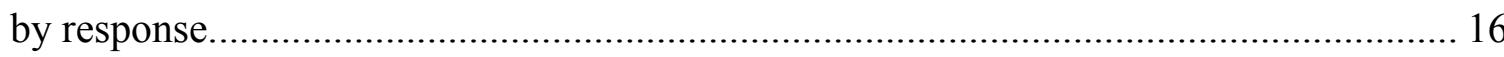

Table 2 Mean arithmetic fluency scores for high and low fluency groups in

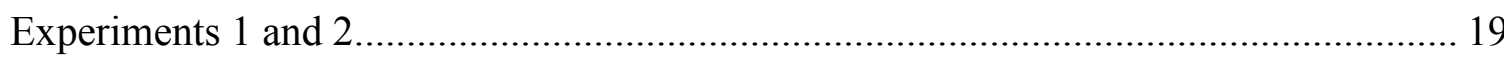

Table 3 Mean response times (in ms), correct trials only, for all 60 participants......... 21

Table 4 Mean response times (in ms), correct trials only, for arithmetic fluency groups consisting of the fastest and slowest 30 participants. ............................................... 23

Table 5 Mean response times (in ms) on unordered sequences, correct trials only, for

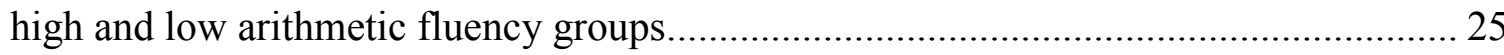

Table 6 Error rates (\%) for arithmetic fluency groups representing a median split of

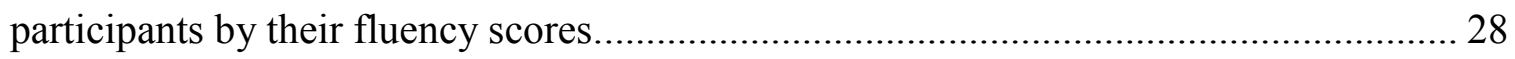

Table 7 Error rates (\%) for unordered "increasing" and "decreasing" sequences by high

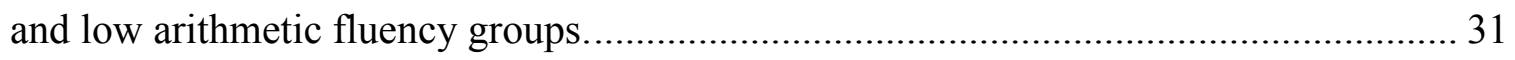

Table 8 Examples of the Six Possible Orders of Digits for each type of sequence...... 44 Table 9 ANOVAs (response times and errors) for Ascending and Descending

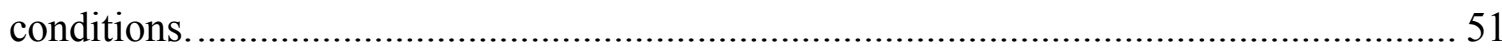

Table 10 ANOVAs (response times and errors) for the Mixed order condition. .......... 60

Table A.1 Complete list of all experimental stimuli used in Experiment 1.................. 92

Table A.2 Complete list of all experimental stimuli used in Experiment 2................. 94 


\section{List of Figures}

Figure 1 Trial format for the ordering task. 18

Figure 2 Ordered and unordered mean response times for participants across all categories.

Figure 3 Ordered and unordered mean response times for high and low fluency

participants across all categories. 22

Figure 4 Unordered "increasing" and "decreasing" mean response times for high and low fluency participants across the counting, balanced, and neutral categories.

Figure 5 Error rates (\%) for ordered and unordered sequences for all categories across all participants

Figure 6 Error rates (\%) for unordered "increasing” and "decreasing" sequences for all

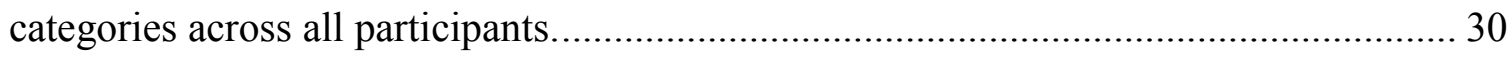

Figure 7 Unordered "increasing”, “decreasing”, and ordered mean response times for all categories across all participants. 37

Figure 8 Trial format for ordering task in Experiment 2 ................................... 48

Figure 9 Experiment 2: Counting, balanced, arithmetic, and neutral mean response times compared across Ascending and Descending order conditions.

Figure 10 Experiment 2: Ordered, "Increasing", and "Decreasing" mean response times and error rates compared across order conditions (Ascending, Descending)..... 53 Figure 11 Experiment 2: Ordered, "Increasing", and "Decreasing" mean response times and error rates compared across all categories 54 Figure 12 Experiment 2: Ordered, "Increasing", and "Decreasing" mean response times for all categories across order conditions (Ascending, Descending) 54 
Figure 13 Experiment 2: Ordered, "Increasing," and "Decreasing" error rates compared across categories and order conditions (Ascending, Descending). 56

Figure 14 Experiment 2: Ordered, "increasing", and "decreasing" mean response times compared across order conditions (Ascending, Descending) and fluency groups 58

Figure 15 Experiment 2: Counting, balanced, arithmetic, and neutral mean response

times and error rates compared across fluency groups in the Mixed condition.

Figure 16 Experiment 2: Ascending, descending, "increasing", and "decreasing"

response times and error rates compared across fluency groups

Figure 17 Experiment 2: Ascending, descending, "increasing", and "decreasing" response times and error rates compared across all categories.

Figure 18 Experiment 2: Ascending, descending, "increasing," and "decreasing" mean response times for both fluency groups across all categories in the Mixed condition 64

Figure 19 Experiment 2: Ascending, descending, "increasing," and "decreasing" error rates for both fluency groups across all categories in the Mixed condition.

Figure 20 Experiment 2: Ascending, descending, "increasing," and "decreasing" mean response times for the counting and neutral categories compared across fluency groups in the Mixed condition 71

Figure 21 Experiment 2: Ascending, descending, "increasing," and "decreasing" error rates for the counting and neutral categories compared across fluency groups in the Mixed condition. 72

Figure 22 Experiment 2: Ordered, "increasing," and "decreasing" mean response times for the counting and neutral categories in the pure order conditions (Ascending, Descending) 
Figure 23 Fluency measure used to calculate participants' arithmetic fluency scores... 99 


\section{INTRODUCTION}

Mental arithmetic is the focus of a large body of cognitive research (e.g., Ashcraft, 1982, 1992; Baroody, 1984; Geary, 1993; DeStefano \& LeFevre, 2004; Prather \& Alibali, 2009; Cohen Kadosh et al., 2013; Zamarian, Ischebeck, \& Delazer, 2009). The ability to perform simple mental computations is an important skill that is involved in activities where using a calculator can be impractical. The management of money, navigation, and measurement are just some examples of situations where the ability to quickly and accurately perform mental calculations are essential. Understanding the processes responsible for arithmetic performance is becoming increasingly important given the consistent decline in mathematical abilities over the last few decades (Mulhern \& Wylie, 2004).

Until recently, a majority of the research on mathematical cognition has been focused on magnitude processing or on arithmetic operations and computations (Ashcraft \& Guilliame, 2009). However, Lyons and his colleagues (Lyons \& Beilock, 2009, 2011; Lyons, Price, Vaessen, Blomert, \& Ansari, 2014) have proposed that ordinal knowledge, as exemplified by the ability to make accurate order judgements of numerical sequences, is a central feature of skilled arithmetic performance. According to Lyons and Beilock $(2009,2011)$, ordinality, or the knowledge of the order relationships that exist between digits, is a primary factor in numerical sequence recognition. They further suggest that individuals with good working memory abilities are more able to perceive and process these ordinal relationships than those with poorer working memory. Lyons and Beilock argue that it is this increased capacity for dealing with ordinal information that enables individuals with good working memory to demonstrate greater arithmetic skill and better 
overall performance than their counterparts who have lower working memory abilities (LeFevre \& DeStefano, 2004; Ragubar, Barnes, \& Hecht, 2010). I hypothesized, in contrast, that the major factor influencing an individual's ability to quickly and accurately recognize numerical sequences is not ordinality, but sequence familiarity, obtained through frequent exposure to these sequences during early learning.

The goal of the present research was to explore the pattern of results reported by Lyons and Beilock and to better understand how ordinal knowledge and sequencefamiliarity is related to arithmetic skill. Determining which factors are of primary importance with respect to sequence recognition will allow researchers and educators to further develop and modify their research and educational practices.

\section{Numerical Cognition}

Numerical representations consist of two interrelated aspects: a representation of quantity or cardinality (Dehaene, 1997) and a representation of relative order or ordinality (Anderson \& Cordes, 2013; Jacob \& Nieder, 2008; Tzelgov \& Ganor-Stern, 2004). Cardinality is a core concept of numerical representation because it reflects "how many" things are in a set, that is, size, number, or quantity. Both young infants and nonhuman animals have demonstrated the ability to discriminate set size differences, though ability to do so improves with both practice and age (Agrillo et al., 2010; Brannon \& Jordan, 2006; Cordes and Brannon, 2008; Emmerton, 1998; Lipton \& Spelke, 2003, 2004; Spelke \& Wood, 2005; Spelke \& Xu, 2000).

Ordinality refers to the ordered relations that exist among the numbers in a sequence (i.e., "what comes next"). Ordinality is therefore an integral aspect of numerical cognition, reflecting relations and relative positions among numerical quantities, such as 
coming before or after one another. This ability to distinguish relative order information is essential to mathematical learning and, like cardinality, is present in non-human animals and infants even early on in their development (Anderson \& Cordes, 2013; Bisazza et al., 2010; Brannon, 2002; Brannon \& Cantlon, 2006; Brannon \& Terrace, 1998, 2002). Therefore, learners need to acquire the basic concepts of cardinality and ordinality and how they interrelate.

\section{Familiarity and Arithmetic Performance}

There is a great deal of evidence to support familiarity as an important factor in mathematical processing. Arithmetic performance is strongly related to how familiar individuals are with the specific operations and procedures that are involved. Many predominant models of mental arithmetic hypothesize that arithmetic facts are stored in an associative network consisting of various number nodes (Ashcraft, 1983, 1987; Butterworth, Zorzi, Girelli, \& Jonckheere, 2001; Campbell, 1995; Campbell \& Oliphant, 1992; Verguts \& Fias, 2005). Solvers often retrieve the answers to arithmetic facts directly from memory (Campbell \& Xue, 2001; LeFevre et al., 1996). In the case of arithmetic problems, the operands as well as the operation symbol all act as cues for memory retrieval. First there is the presentation of an arithmetic problem (e.g., $3+4)$, which results in the activation of the number nodes specified in the problem (e.g., 3 and 4). Activation then spreads from these presented nodes along associative links so that related number nodes, such as the sum, are also activated (Ashcraft, 1987; LeFevre et al., 1988). The rapid and automatic retrieval of these stored arithmetic facts reflects strong familiarity with the numerical problems themselves. Learning multiplication tables is a prime example. Children practice those tables until they are familiar enough with the 
multiplication problems that they can directly, and almost immediately, retrieve the answers from memory, no longer needing to perform mental computations to obtain the correct response (Lemaire \& Siegler, 1985). Therefore, the automatic and speedy retrieval of information implies a significant degree of familiarity with the information itself. Otherwise, additional mental processing would be required before the correct information can be obtained.

Given that activation of the associative network is automatic and obligatory, it can lead to the creation of interference effects due to competing node information (LeFevre et al., 1988). For example, this interference effect can occur in arithmetic problems, such as $5+4$. The nodes responsible for the addition operator and the operands 5 and 4 will activate (i.e., $5+4=9$ ), as expected, however the node for multiplication could also activate $(5 \times 4=20)$ and thus provide conflicting information, resulting in longer reaction times and/or erroneous responses. Exactly how this information conflict is resolved is unclear, but the predominant view suggests it is facilitated by the influx of appropriate information, inhibition of inappropriate information, or a combination of the two (Hasher, Zacks, \& May, 1999; MacLeod et al., 2003).

The importance of inhibition in effectively utilizing the associative network of arithmetic facts suggests that individual differences in inhibitory control could greatly influence arithmetic performance. Accordingly, several research studies have indicated that less-skilled arithmetic problem solvers demonstrate a greater tendency for making intrusion errors and have lower scores on working memory tasks that require inhibition of irrelevant information, than their more-skilled counterparts (e.g., D'Amico \& Passolunghi, 2009; Geary, Hoard, \& Bailey, 2012; Passolunghi \& Siegel, 2001). 
Therefore, effective inhibition of irrelevant information is important for accurate and speedy arithmetic problem solving and it could lead to highly-skilled individuals exhibiting smaller interference effects and making fewer errors than their less-skilled counterparts on equally familiar stimuli.

\section{Factors Influencing Numerical Sequence Recognition}

During the early stages of numerical development in children, prior to the formation of an associative network of arithmetic facts, they develop sequential associations (i.e., the counting-string relations). Through counting, children understand and recognize the relationships between different numbers and where they belong in the sequence (e.g., 3 comes before 4, which is followed by the number 5; Siegler \& Shrager, 1984). Siegler and Shrager suggested that this system of sequential associations evolves into the more complex associative network of arithmetic facts through feedback and practice with arithmetic problems and their solutions. For example, children around the age of 5 may respond to $3+4$ with the answer 5 , reflecting strong links between counting sequences and responses. However, as children practice arithmetic facts, the association between arithmetic problems and their correct answers become stronger than their associations based on counting-string relations (LeFevre \& Kulak, 1991). Regardless, these sequential associations remain strong, as evidenced by distance effects, where both children and adults have difficulty rejecting digits that are numerically close to a target digit (e.g., 6 is more difficult to reject than 4 when the target is 7) in recognition tasks (e.g., Holloway \& Ansari, 2009). Furthermore, counting strategies are one of the nonretrieval strategies that both children and adults use in order to solve arithmetic problems (Campbell \& Xue, 2001; LeFevre, DeStefano, Penner-Wilger, \& Daley, 2006). These 
findings indicate that numerical sequence knowledge, particularly of highly familiar counting strings, is related to arithmetic performance. Although some researchers currently view individual differences in the ability to detect ordinality as the causal factor in sequence recognition (Lyons \& Beilock, 2009, 2011), familiarity can potentially provide a much more complete account of the response patterns associated with sequence recognition findings.

In Lyons and Beilock (2009, Experiment 2), participants were shown 3-digit numerical sequences and had to decide whether or not they were in ascending order. Three different types of number sequences were used: counting (e.g., 12 3), balanced (e.g., 24 6), and neutral (e.g., 127$)^{1}$. Counting stimuli were assumed to have the greatest degree of ordinal association between the constituent digits (due to the obvious pattern and proximity of the digits). Neutral stimuli had the weakest degree of ordinal association (no visible pattern between the digits), balanced stimuli fell somewhere in between the two extremes (i.e., a consistent pattern is present, but with increments greater than 1). Participants responded more slowly to unordered than to ordered sequences, but this general result varied across sequence type and whether the participants had high or low working memory ability. The largest difference between ordered and unordered sequences was found for the counting sequences and was shown by both high and low working memory groups. Neither group showed a difference between ordered and unordered stimuli for neutral sequences. However, participants with low working memory responded to balanced stimuli more like neutral stimuli, whereas participants

\footnotetext{
${ }^{1}$ It should be noted that Lyons and Beilock used different category labels in their research. For simplicity, all label references will use the labelling system put forth in the current paper, (e.g., Counting, Balanced, and Neutral).

${ }^{2}$ A typing error resulted in the added repetition of one balanced sequence and one less
} 
with high working memory treated balanced stimuli more like counting stimuli. Lyons and Beilock suggested that high working memory participants had a greater ability to perceive and process the ordinal relationships between digits in balanced sequences. Furthermore, working memory skill has been found to be strongly related to arithmetic performance (DeStefano \& LeFevre, 2004; LeFevre et al., 2005). Therefore, Lyons and Beilock inferred that individuals with high working memory displayed superior arithmetic performance because they were better at processing ordinal information.

In apparent support of their conclusions, Lyons and Beilock (2011) found a very strong correlation between participants' decision times on these three-digit sequences and their solution performance on multi-digit calculations (e.g., $43+79,18 \times 5)$. Although they suggested that this relation reflects the importance of ordinal knowledge for calculation, there is at least one alternative explanation for this correlation. The strong correlation can be explained by the relatively better (faster) performance of the moreskilled participants on what Lyons and Beilock (2009) called balanced sequences. Balanced sequences are those with constant differences between the numbers, such as 25 8, 35 7, and 15 9. As shown by LeFevre and Bisanz (1986), individuals with better calculation skills are faster than those with poorer skills on balanced sequences that are less familiar (e.g., 369 vs. 25 8). Lyons and Beilock (2009) similarly found that individuals with better working memory ability also recognized more balanced sequences. Thus, rather than concluding that faster responding to balanced sequences was necessarily due to participants having greater ordinal processing ability, familiarity with the number sequences themselves is another factor that could affect the speed of sequence recognition. 
Familiarity as an influential factor in number recognition and mental arithmetic has been demonstrated in previous research. Lochy et al. (2000) examined the odd-even effect in multiplication, that is, false products are rejected faster when they violate the expected parity (i.e. even $\times$ even $=$ even, odd $\times$ odd $=$ odd, even $\times$ odd $=$ even $)$. Although several researchers (Krueger, 1986; Lemaire \& Fayol, 1995) had largely attributed this effect to the use of a parity rule, Lochy et al. suggested that a familiarity hypothesis was a more viable explanation. In support of their familiarity hypothesis, Lochy et al. found that incorrect even answers (e.g., 9 x $7=62$ ) were rejected more slowly than incorrect odd answers (e.g., 8 x 9=73), even when the two operands were odd. In contrast, according to the parity rule, observing an even answer when the two operands were odd should result in a faster rejection. Instead an interference effect was observed, with even answers being significantly slower to reject than odd ones across all problem types (i.e., average response latencies for odd answers were $121 \mathrm{~ms}$ faster than for even answers), consistent with the higher frequency of even than odd products (i.e., $75 \%$ of products for simple multiplication are even).

Although the familiarity hypothesis does not definitively exclude the possibility that a rejection rule (in this case a parity rule), was being implemented, if use of a parity rule was the determining factor in verifying answer accuracy, then all the incongruent answers should have been rejected faster than congruent answers. However, because even answers were always faster than odd answers, Lochy et al. concluded that the greater familiarity of even product reflects the higher proportion of even products in the multiplication tables. Similarly, in Lyons and Beilock's $(2009,2011)$ research, it may not be some types of ordinal knowledge (in the abstract sense) that are important in the 
connection between the task and calculation, but instead that individuals who are better at calculation essentially have more automatic access to a larger set of sequences, which presumably (at least in part) is a consequence of their practice with arithmetic and numerical tasks in general.

\subsection{The Current Research}

The purpose of the present research was to determine if familiarity, and not ordinality, was primarily responsible for the differential patterns of responding that are observed when participants are mentally processing numerical sequences and classifying them as either ordered or unordered. Although Lyons and Beilock $(2009,2011)$ have argued that individuals with higher working memory possess greater ordinal processing ability and thus demonstrate better sequence recognition, other researchers (LeFevre \& Bisanz, 1986; Lochy et al., 2000) have found that familiarity is an influential factor in numerical processing. The current research will endeavor to determine whether sequence familiarity provides a better explanation than ordinality for the response patterns found by Lyons and Beilock and provide more information about how individuals process number sequences.

The methodology of the current experiments was modelled after Experiment 2 in Lyons and Beilock (2009). However, instead of employing a working memory measure to index individual differences, an arithmetic fluency measure was used. The resulting response patterns were expected to remain consistent with those of Lyons and Beilock, given that arithmetic skill and working memory are strongly correlated (LeFevre et al., 2005). In addition, a new category of number sequence, referred to as arithmetic sequences, was introduced. This fourth category contained number sequences that at first 
glance appeared to be neutral sequences (no obvious inter-digit pattern is visible), but actually reflect an arithmetic relationship (e.g., 145 --> $1+4=5$ ). These sequences do not demonstrate a strong ordinal relationship (as defined by Lyons and Beilock) like those of counting or balanced sequences. Therefore, if ordinality is the determining factor in sequence recognition, then participants should respond to arithmetic sequences as if they were neutral sequences. However, arithmetic sequences should be more familiar than, for example, a neutral sequence, since individuals repeatedly practice and are exposed to these sequences during early learning. Therefore, if familiarity is the determining factor, then these sequences should reflect response patterns that are more similar to counting sequences.

Previous studies have shown that participants with high arithmetic fluency were more efficient in executing both retrieval and procedural strategies to solve addition and multiplication problems than less-fluent participants (Hecht, 1999; LeFevre, Bisanz, et al., 1996; LeFevre, Sadesky, \& Bisanz, 1996). Therefore, people who quickly recognize a range of number sequences should also perform quickly on simple arithmetic problems. Counting sequences (e.g., 12 3) should be very familiar to everyone as they are among the earliest learned and the most often practiced. People should also be familiar with arithmetic sequences (e.g., 134 ), due to having had repetitive practice with these simple computations during learning (LeFevre, Mrkonjic, \& Bisanz, 1988). Similar to what was seen in Lyons and Beilock (2009; LeFevre \& Bisanz, 1986), it was expected that people with greater arithmetic fluency would respond more quickly to balanced sequences (e.g., 246 ), where the differences between the digits are equal to the initial digit, than to neutral sequences (e.g., 26 7). However, balanced sequences such as 258 , where the 
increment is not equal to the initial digit, are less familiar than counting or arithmetic sequences. Therefore, the faster responding of highly skilled participants would occur as a result of more practice with arithmetic and greater exposure to a wider range of number sequences.

Responses for ordered counting sequences, such as 123 , were expected to be faster than unordered ones, such as 213 . Based on the assumption that activation of familiar sequences is highly automatic, it was expected that in addition to faster responding for these familiar sequences, people would have difficulty rejecting unordered number sequences that closely resembled highly familiar ordered sequences. Therefore, response times for unordered counting and arithmetic sequences should be significantly longer than for unordered neutral sequences, which due to relative unfamiliarity should not generate an interference effect. Balanced sequences were hypothesized to be more familiar to high fluency individuals and less familiar to low fluency individuals (LeFevre \& Bisanz, 1986). Therefore, interference effects for unordered balanced sequences should only be present for participants with relatively high arithmetic skill.

Although Lochy et al. (2000) demonstrated that familiarity provided a better explanation for participant response time patterns in multiplication verification than plausibility processing in the form of a parity rule, research by Lemaire and Fayol (1995) indicated that the implementation of strategies or rejection rules (such as a parity rule), can be affected by whether or not a problem is considered easy or difficult. For easy problems, the correct answer is retrieved from memory and an individual's decision is based on that retrieved answer rather than on a plausibility process. However, plausibility processes and other strategies become increasingly exploited as the problem difficulty 
increases. Accordingly, Lochy et al. (2000) also found that parity effects were greater for larger and more difficult problems. Potential strategy implementation in the current study was thus examined, in order to determine if the experimental design yielded any opportunities for a rejection rule to form (such as the greater proportion of even answers in the Lochy et al. study). Although several minor possibilities were found, the most likely strategy that could be exploited in Experiment 1 was determined to be that participants could more quickly reject unordered sequences when the first two digits were in decreasing order (e.g., 312 vs. 13 2). Because participants were required to indicate whether numerical sequences were in increasing order or not, it thus became possible to formulate a rejection rule such as "if the first two digits are decreasing, respond unordered". Use of this rule might allow faster rejection of sequences and even preclude full examination of all three digits of the sequence.

To summarize, there are five main hypotheses for Experiment 1:

1) Participants should respond fastest to ordered counting sequences, because these are most familiar, and slowest to their unordered counterparts, because of interference;

2) Participants should show a response pattern for arithmetic sequences $\left(2^{\text {nd }}\right.$ most familiar) that is similar to counting sequences, but less pronounced because the degree of familiarity (and thus interference) is less;

3) Participants should respond equally quickly to ordered and unordered neutral sequences (i.e., no interference); 
4) High-fluency participants should treat balanced sequences more like counting/arithmetic sequences (interference effects), whereas low fluency participants should treat them more like neutral sequences (no interference effects);

5) Participants should display a significant response time advantage for unordered "decreasing" sequences, while being significantly slower for unordered "increasing" sequences. 


\section{EXPERIMENT 1}

\section{Method}

\section{Participants}

Sixty participants (37 female and 23 male) were recruited for Experiment 1, with $95 \%$ of the sample being under 40 years of age (age: $18-67$ years; $M=24$ years; $M d n=$ 21 years). The data from one additional participant was excluded from the study due to his ill health and inability to maintain focus during the ordering task. All participants completed two tasks: an arithmetic fluency measure and the sequence ordering task. Materials

The fluency measure was a paper-and-pencil test of complex arithmetic (see

Appendix B). The test had three components: one page with complex addition problems, another page with complex subtraction problems, and a final page of multiplication problems. Participants were given one minute per page and were instructed to solve the problems as quickly and accurately as possible. The correct answers on all sections were summed to yield a total score of arithmetic fluency.

The ordering task was conducted using a Mac computer. Stimuli were displayed at 1280 x 1024-resolution on a 19.1" Mac flat-panel monitor, approximately $61 \mathrm{~cm}$ from the participant. On each trial, participants saw a horizontal sequence of three, 1-digit Arabic numerals, with integers ranging from 1 to 9 . Stimuli were in white 72-point Courier font presented on a black background. The distance between the left and rightmost numerals subtended $\sim 5.1^{\circ}$ of visual angle. Numerals were separated from one another by $\sim 1.9^{\circ}$ of visual angle. 
The ordering task required participants to indicate whether the 3-digit sequences were in ascending order from left to right by pressing one of two buttons on an RB-730 response pad. There were a total of seven buttons on the response box: two coloured (the response keys) and the rest white (not used during the experiment). In keeping with Lyons and Beilock's experimental procedure, the two response keys were the same distance apart as the "Z" and " $\mathrm{M}$ " keys on a standard keyboard. If all three digits were in ascending order, participants were to press the green button, on the left side of the response box. If the sequences were not in order, or were in descending order from left to right, participants were instructed to press the blue button on the right side of the response box. Importantly, participants had to have their hands positioned with a finger on each response button, so that they could immediately press the appropriate button once a decision had been made.

\section{Design}

Four different stimulus categories were used in the ordering task: counting, balanced, arithmetic and neutral. When properly ordered (i.e., on "ordered" trials), counting stimuli formed a three-digit segment of the integer count sequence (e.g., 123 ). Balanced stimuli, when correctly ordered, formed a pattern with constant intervals between adjacent numbers (e.g., 24 6). Arithmetic stimuli, when properly ordered, formed a sequence where the sum of the first two numbers in the sequence yielded the last number (e.g., 134 ). Neutral stimuli, when correctly ordered, formed a pattern with unequal intervals between adjacent numbers (e.g., 16 8). Unordered sequences were formed by randomly rearranging the ordered sequences so that they were no longer in order (see Table 1). 
Table 1. Sample ordered and unordered sequences for each category in the ordering task by response. Ordered trials indicate that the stimuli were in ascending order; unordered trials had the same digits, but were not ordered by digit size.

\begin{tabular}{cccccccc}
\hline \multicolumn{2}{c}{ Counting } & \multicolumn{2}{c}{ Balanced } & \multicolumn{2}{c}{ Arithmetic } & \multicolumn{2}{c}{ Neutral } \\
\hline Ordered & Unordered & Ordered & Unordered & Ordered & Unordered & Ordered & Unordered \\
\hline 123 & 132 & 135 & 153 & 134 & 143 & 138 & 183 \\
234 & 423 & 246 & 462 & 257 & 572 & 237 & 372 \\
345 & 354 & 357 & 375 & 358 & 385 & 359 & 395 \\
456 & 465 & 468 & 486 & 459 & 495 & 479 & 497 \\
\hline
\end{tabular}

The task consisted of 352 trials. There were 56 counting sequences ( 28 ordered, 28 unordered), $73^{2}$ balanced sequences ( 37 ordered sequences and 36 unordered), 48 arithmetic sequences ( 24 ordered, 24 unordered), and 175 neutral sequences ( 87 ordered, 88 unordered). The 352 trials were split up into 4 equivalent blocks, each having equal numbers of each category (i.e., counting, balanced, arithmetic, neutral) and sequenceorder (i.e. ordered, unordered). Within each block, these 88 trials would be randomly presented to the participant. Due to the limited number of possible ordered sequences for the counting, arithmetic, and balanced categories, some sequences were repeated: ordered sequences in the counting and balanced categories were repeated four times, whereas ordered arithmetic and neutral sequences were repeated twice. There was a much greater range of possible unordered sequences and ordered neutral sequences, thus a random assortment of the different possible arrangements was selected. The unordered sequences

\footnotetext{
${ }^{2}$ A typing error resulted in the added repetition of one balanced sequence and one less repetition of a neutral sequence. Given that there are multiple repetitions of each stimulus, the effect is likely negligible.
} 
in the counting and balanced categories were repeated twice, while unordered arithmetic sequences were repeated four times and the neutral sequences were not repeated. Examples are shown in Table 1. The full set of stimuli is shown in Appendix A.

\section{Procedure}

In a single testing session, participants were asked to complete two tasks related to the current study: the fluency measure followed by the number sequence ordering task. At the start of the session, participants were given the fluency measure and told that they had to complete the task as quickly and accurately as possible, within a time-limit of one minute per page. After they had completed the fluency measure, participants were informed that they were to perform a number sequence ordering task, where they would have indicate whether 3-digit sequences were in ascending order ("ordered") or not ("unordered"). In order to help participants become more familiar with the task, they were all given the same 10 practice trials, with accompanying feedback regarding the accuracy of their responses. The 10 practice trials involved two counting (one ordered and one unordered), two balanced (one ordered and one unordered), two arithmetic (one ordered and one unordered), and four neutral (two ordered and two unordered) sequences.

After the practice trials there was a break, allowing participants to have time to ask questions and giving the experimenter the opportunity to ensure their understanding of the task. Once ready, participants moved on to the experimental trials, where no further feedback about the individual trials was provided.

For each trial, the three one-digit Arabic numerals were presented on the screen for a maximum of $1500 \mathrm{~ms}$. If participants had not responded by this time, the stimuli vanished, leaving only the black background until they did. Whenever a response was 
detected, three vertical white bars appeared at the center of the screen for a fixed interstimulus interval of $1500 \mathrm{~ms}$. These bars acted as both a fixation point and an indicator for participants to know that their response had been received. An example trial is shown in Figure 1.

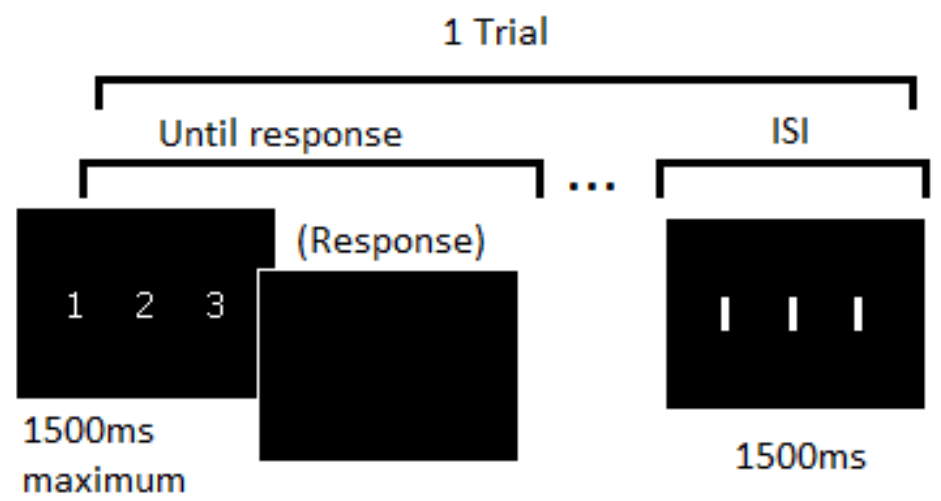

Figure 1. Trial format for the ordering task. Stimuli were presented for a maximum of 1500 ms. If participants responded before this time limit, the sequence immediately moved to the inter-stimulus-interval (ISI); otherwise, stimuli disappeared after $1500 \mathrm{ms,}$ leaving a blank screen, which remained until participants responded.

\section{Results}

Individual differences in arithmetic fluency were determined by the total score (number correct) obtained on the arithmetic fluency task. The maximum possible score across all three pages was 180 . A median split of the 60 total participants was used to assign participants with the highest 30 and lowest 30 fluency scores to high and low arithmetic fluency groups, respectively (see Table 2). Sowinski et al. (2014) reported mean scores around 30 for Canadian-educated participants and means scores around 50 for Chinese-educated participants. 
Table 2. Mean arithmetic fluency scores for high and low fluency groups in Experiments 1 and 2.

\begin{tabular}{cccccccc}
\hline & \multicolumn{3}{c}{ Experiment 1} & & \multicolumn{3}{c}{ Experiment 2 } \\
\cline { 1 - 3 } \cline { 6 - 8 } Fluency & $M$ & $S D$ & $n$ & & $M$ & $S D$ & $n$ \\
\hline Low & 28.2 & 7.0 & 30 & & 25.0 & 5.2 & 39 \\
High & 55.5 & 12.4 & 30 & & 49.2 & 17.6 & 39 \\
\hline
\end{tabular}

Data screening was conducted prior to data analyses (for more detailed information regarding data screening, see Experiment 2, page 58). Due to the inherent skewness of response time data, median (instead of mean) ordered and unordered scores were calculated for each category per participant (incorrect trials were excluded from all reaction time analyses). Latencies and percentage of errors were analyzed and discussed separately. Data were analyzed in separate 2(fluency: low, high) x 4(category: counting, balanced, arithmetic, neutral) x 2(sequence-order: ordered, unordered) mixed ANOVAs, with sequence-order and category as repeated measures factors and fluency as a betweengroups factor. Post hoc testing was conducted using 95\% confidence intervals based on the mean square error for each significant interaction (Masson \& Loftus, 2003). These confidence intervals were displayed as error bars in each data figure, with no overlap indicating statistically significant differences.

\section{Response Time}

High-fluency participants responded faster than low-fluency participants (804 ms vs. $1056 \mathrm{~ms}), F(1,58)=22.16, p<.001, \eta_{\mathrm{p}}{ }^{2}=.276$. Latencies also differed by sequenceorder such that participants responded more quickly on ordered than on unordered trials $(898 \mathrm{~ms}$ vs. $962 \mathrm{~ms}), F(1,58)=33.93, p<.001, \eta_{\mathrm{p}}{ }^{2}=.369$. Latencies also varied by category $($ counting $=953 \mathrm{~ms}$, balanced $=907 \mathrm{~ms}$, arithmetic $=952 \mathrm{~ms}$, neutral $=909 \mathrm{~ms})$, $F(3,174)=17.13, p<.001, \eta_{\mathrm{p}}^{2}=.228$ 
Notably, there were no significant two-way interactions between fluency and category or fluency and sequence-order, $F \mathrm{~s}<1$. However, there was a significant twoway interaction between category and sequence-order, $F(3,174)=41.93, p<.001, \eta_{\mathrm{p}}{ }^{2}=$ .420 , with both counting and arithmetic sequences showing differences between ordered and unordered sequences, whereas balanced and neutral did not (see Table 3 for means). As shown in Figure 1.5, participants showed large interference effects for counting sequences (most familiar), with slow latencies on unordered sequences compared to ordered sequences. In contrast, response latencies for ordered and unordered neutral sequences (least familiar) were essentially identical.

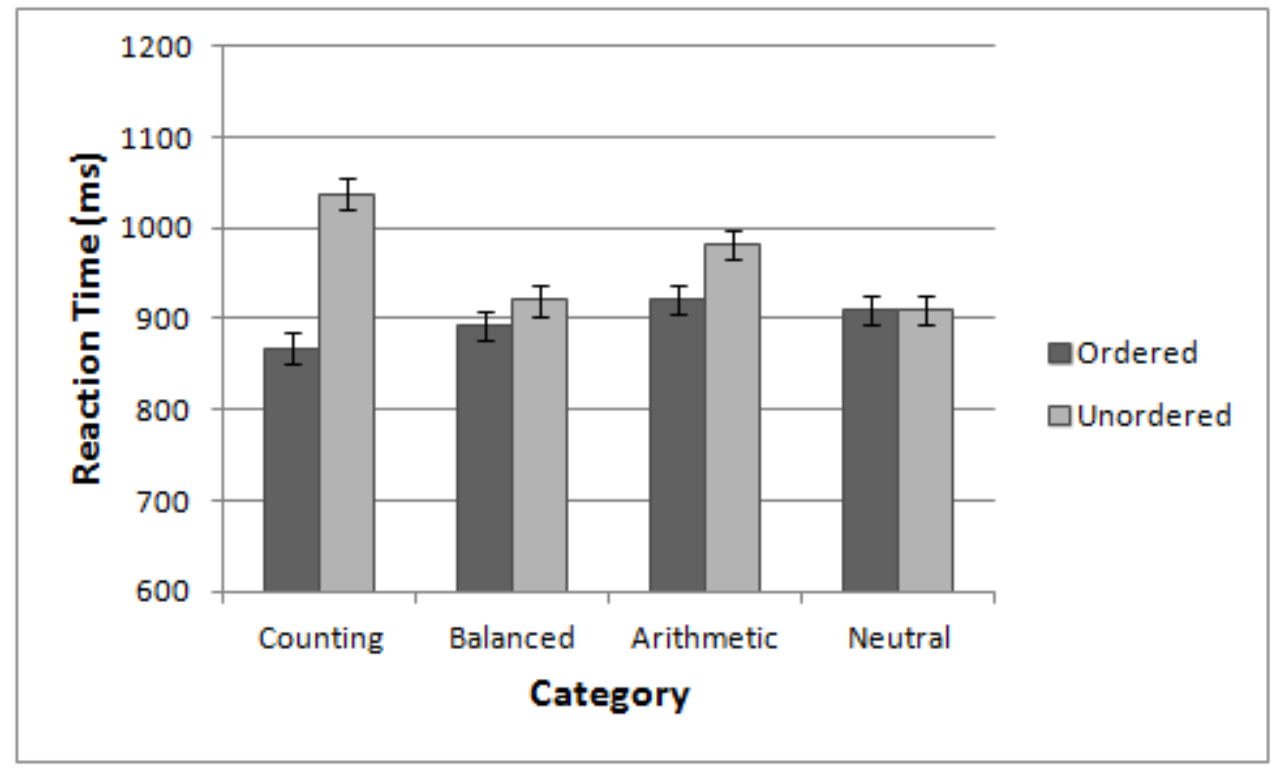

Figure 2. Ordered and unordered mean response times for participants across all categories. 
Table 3. Mean response times (in ms), correct trials only, for all 60 participants.

\begin{tabular}{|c|c|c|c|c|c|c|c|c|}
\hline \multirow{3}{*}{ Response } & \multicolumn{8}{|c|}{ Stimulus Category } \\
\hline & \multicolumn{2}{|c|}{ Counting } & \multicolumn{2}{|c|}{ Balanced } & \multicolumn{2}{|c|}{ Arithmetic } & \multicolumn{2}{|c|}{ Neutral } \\
\hline & $M$ & $S E$ & $M$ & $S E$ & $M$ & $S E$ & $M$ & $S E$ \\
\hline Ordered & 868 & (30) & 893 & (26) & 921 & (31) & 909 & (28) \\
\hline Unordered & 1038 & (32) & 920 & (26) & 983 & (29) & 909 & (24) \\
\hline
\end{tabular}

The overall ANOVA did not reveal a significant three-way interaction among fluency, category, and order, $F(3,174)=.82, p=.487, \eta_{\mathrm{p}}{ }^{2}=.014$. However, because Lyons and Beilock (2009) found differential patterns on balanced sequences for high- and low-skilled participants (based on working memory skill), I explored the patterns for balanced sequences in the present experiment (see Table 4 for means). As shown in Figure 3, performance across skill groups was similar for counting, arithmetic, and neutral sequences, whereas on balanced sequences, there was a significant difference between balanced ordered and unordered sequences for only high fluency participants, $t(29)=2.88, p<.01$, which was consistent with the results of Lyons and Beilock (2009).

These results indicated that participants in both fluency groups responded similarly to counting, neutral and arithmetic stimuli, and low-fluency individuals treated balanced stimuli more like neutral stimuli, in that there was no significant difference between ordered and unordered response times. In contrast, high fluency individuals treated balanced stimuli more like counting stimuli, showing a similar interference bias towards unordered responses as shown on counting sequences, and suggesting a greater 
familiarity with this type of sequence. Note that the size of this interference effect was much smaller than that shown on counting sequences, however.

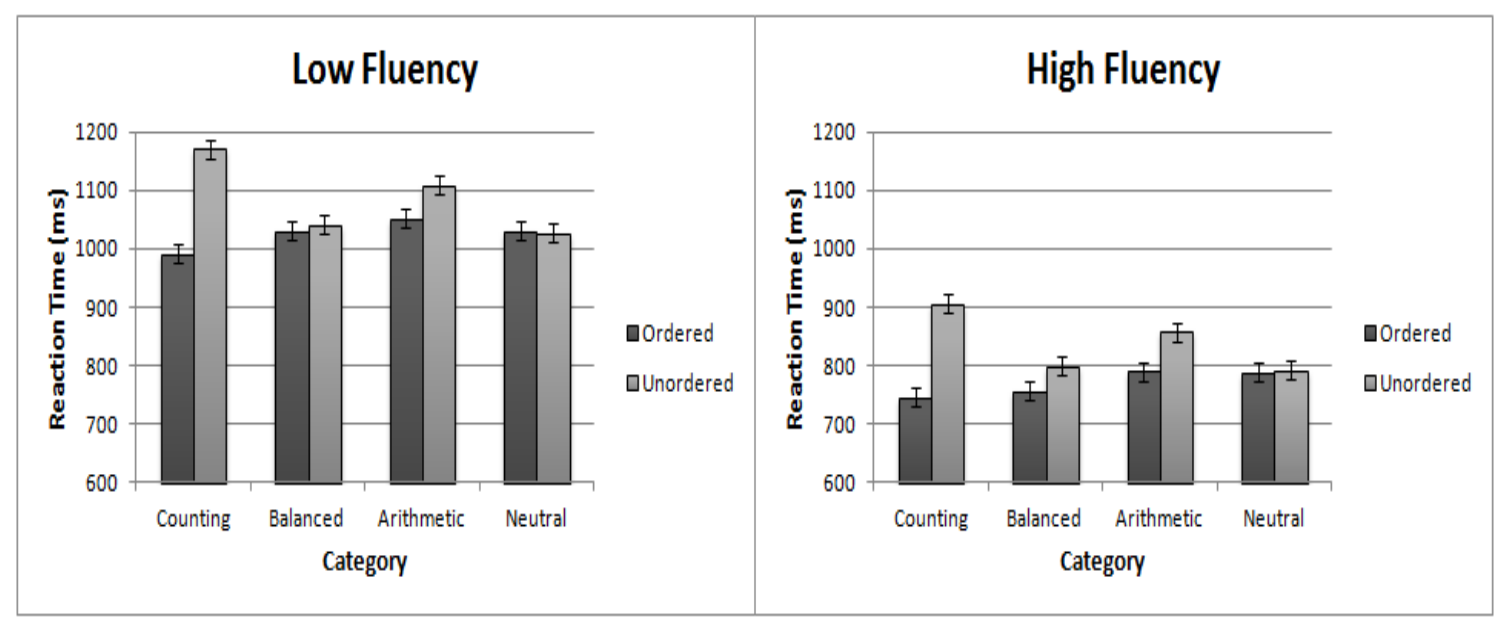

Figure 3. Ordered and unordered mean response times for high and low fluency participants across all categories.

Both high- and low-skill groups showed significant differences between ordered and unordered arithmetic sequences, suggesting that the digits in these sequences resulted in obligatory activation even when the sequences were not ordered. However, additional analyses of the unordered sequences (described below) suggested that this difference between ordered and unordered sequences was not due to familiarity and so these results will be interpreted after those findings have been described.

Notably, less-skilled participants responded faster to ordered counting sequences than they did to any other type of sequence and both fluency groups responded faster to ordered counting sequences than to their ordered neutral versions. This pattern suggested that although low-fluency participants may be slower at recognizing and processing information than high fluency participants, they still have a great deal of practice with counting sequences and thus recognition is fairly quick and highly automated. Moreover, this familiarity explains why it is so difficult for the two groups to reject unordered counting sequences. 
Table 4. Mean response times (in ms), correct trials only, for arithmetic fluency groups consisting of the fastest and slowest 30 participants.

\begin{tabular}{|c|c|c|c|c|c|c|c|c|}
\hline \multirow{3}{*}{ Response } & \multicolumn{8}{|c|}{ Stimulus Category } \\
\hline & \multicolumn{2}{|c|}{ Counting } & \multicolumn{2}{|c|}{ Balanced } & \multicolumn{2}{|c|}{ Arithmetic } & \multicolumn{2}{|c|}{ Neutral } \\
\hline & $M$ & $S E$ & $M$ & $S E$ & $M$ & $S E$ & $M$ & $S E$ \\
\hline & \multicolumn{8}{|c|}{ High fluency } \\
\hline Ordered & 745 & (42) & 756 & (37) & 791 & (44) & 788 & (39) \\
\hline \multirow[t]{2}{*}{ Unordered } & 906 & $(45)$ & 799 & $(36)$ & 858 & (41) & 791 & (34) \\
\hline & \multicolumn{8}{|c|}{ Low fluency } \\
\hline Ordered & 991 & (42) & 1030 & (37) & 1051 & (44) & 1031 & (39) \\
\hline Unordered & 1171 & $(45)$ & 1041 & $(36)$ & 1108 & (41) & 1026 & (34) \\
\hline
\end{tabular}

To further explore the cognitive processes involved in the sequence task, a separate analysis was conducted on participants' reaction times to unordered sequences. The sequences were categorized according to the pattern in the first two digits. If the first two digits were in ascending order (e.g., 23 1), the sequence-order was categorized as "increasing". If the first two digits were in descending order (e.g., 213 ), the sequence was categorized as "decreasing". If participants used the pairwise relations between the digits to develop a strategy for determining if sequences were ordered, then participants should more quickly reject unordered sequences when the first two digits were decreasing, given that it violates the ascending rule (i.e. $1^{\text {st }}$ digit $<2^{\text {nd }}$ digit $<3^{\text {rd }}$ digit), 
thus helping to rule out those sequences. Arithmetic sequences could not be included in this analysis because unordered arithmetic sequences were only of the "increasing" type. ${ }^{3}$

The median response times for unordered sequences were entered into a 2(fluency: low, high) x 3(category: counting, balanced, neutral) x 2(sequence-order: increasing, decreasing) mixed ANOVA with sequence-order and category as repeated measures factors and fluency as a between-groups factor. See Table 5 for means.

In accord with the first ANOVA analysis, high-fluency participants responded faster to unordered sequences than low-fluency participants (835 ms vs. $1083 \mathrm{~ms}), F(1$, $58)=22.14, p<.001, \eta_{\mathrm{p}}{ }^{2}=.276$. As seen in Figure 4, high fluency participants responded more quickly than low fluency participants across all three categories and sequence-order types. Latencies also differed by sequence-order, with participants responding faster to "decreasing" unordered sequences than to "increasing" unordered sequences (924 ms vs. $994 \mathrm{~ms}), F(1,58)=42.94, p<.001, \eta_{\mathrm{p}}{ }^{2}=.425$. Latencies also varied by category $($ counting $=1046 \mathrm{~ms}$, balanced $=918 \mathrm{~ms}$, neutral $=911 \mathrm{~ms}), F(2,116)$ $=98.98, p<.001, \eta_{\mathrm{p}}{ }^{2}=.631$, with unordered counting sequences showing significantly longer response times than either balanced or neutral unordered sequences.

\footnotetext{
${ }^{3}$ Unordered "decreasing" arithmetic sequences were deliberately excluded (e.g., for the sequence 134 , neither 314 nor 413 were used; the former preserves the arithmetic relation $3+1=4$ and the latter is a subtraction relation $4-1=3$ ).
} 
Table 5. Mean response times (in ms) on unordered sequences, correct trials only, for high and low arithmetic fluency groups.

Stimulus Category

\begin{tabular}{|c|c|c|c|c|c|c|}
\hline \multirow[t]{2}{*}{ Sequence-order } & \multicolumn{2}{|c|}{ Counting } & \multicolumn{2}{|c|}{ Balanced } & \multicolumn{2}{|c|}{ Neutral } \\
\hline & $M$ & $S E$ & $M$ & $S E$ & $M$ & $S E$ \\
\hline & \multicolumn{6}{|c|}{ Low fluency } \\
\hline Increasing & 1210 & $(45)$ & 1084 & $(36)$ & 1055 & (36) \\
\hline \multirow[t]{2}{*}{ Decreasing } & 1159 & (49) & 984 & (37) & 1002 & (35) \\
\hline & \multicolumn{6}{|c|}{ High fluency } \\
\hline Increasing & 953 & $(45)$ & 841 & $(36)$ & 819 & $(36)$ \\
\hline Decreasing & 864 & (49) & 763 & (37) & 770 & $(35)$ \\
\hline
\end{tabular}

No significant two- or three-way interactions were found among sequence-order, category, or fluency, $p s>.05$. These results suggest that although participants are using a rejection rule to more rapidly process numerical sequences, implementation of this rule was comparable for high and low fluency individuals. Therefore, when faced with an unordered sequence where the two first digits were in decreasing order, both high and low-skilled participants were able to more quickly and easily classify that sequence than if the first two digits were in increasing order. Conversely, when faced with an unordered sequence where the two first digits were increasing, a thorough examination of the entire digit string was required before a decision can be made.

As expected, "increasing” counting sequences showed significantly greater latencies than for either balanced or neutral. However, "decreasing" counting sequences 
were similarly found to have longer response times than either balanced or neutral sequences (see Figure 3). Although it was expected that "increasing" counting sequences would demonstrate strong interference effects due to their level of familiarity, it was not anticipated that "decreasing" sequences would also display an interference effect, since a truly self-terminating strategy would dictate that processing ends as soon as an ascending rule violation is detected (in which case 534 should be equivalent to 539 , since both start with 5 3). Evidently some residual processing of the $3^{\text {rd }}$ digit was still taking place, potentially as some sort of check, which would be consistent with a fast and automatic (obligatory) activation of the counting sequence. One possible explanation given by Eriksen and his colleagues for this residual processing is that an individual's strategic control is potentially quite limited and even if the necessity of searching for additional information is eliminated, some processing of non-target items is unavoidable, particularly when the stimulus set is small (Colegate, Hoffman, \& Eriksen, 1973; Eriksen \& Collins, 1969; Eriksen \& Hoffman, 1972; Eriksen \& Rohrbaugh, 1970), such as in the current study. Therefore, although a self-terminating strategy offers a better explanation than a fully exhaustive processing strategy for why participants responded more quickly to "decreasing" sequences than to "increasing" sequences, the robust interference effect displayed by the "decreasing" counting sequences indicated that at least partial processing of the third digit did occur and was sufficient to cause obligatory activation. Therefore, the greatest contribution of the rule violation may be that it served as an extra cue or added confirmation that a sequence was unordered, thus speeding up participants' decision times and enhancing their ability to overcome interference, rather than reducing incoming information. 


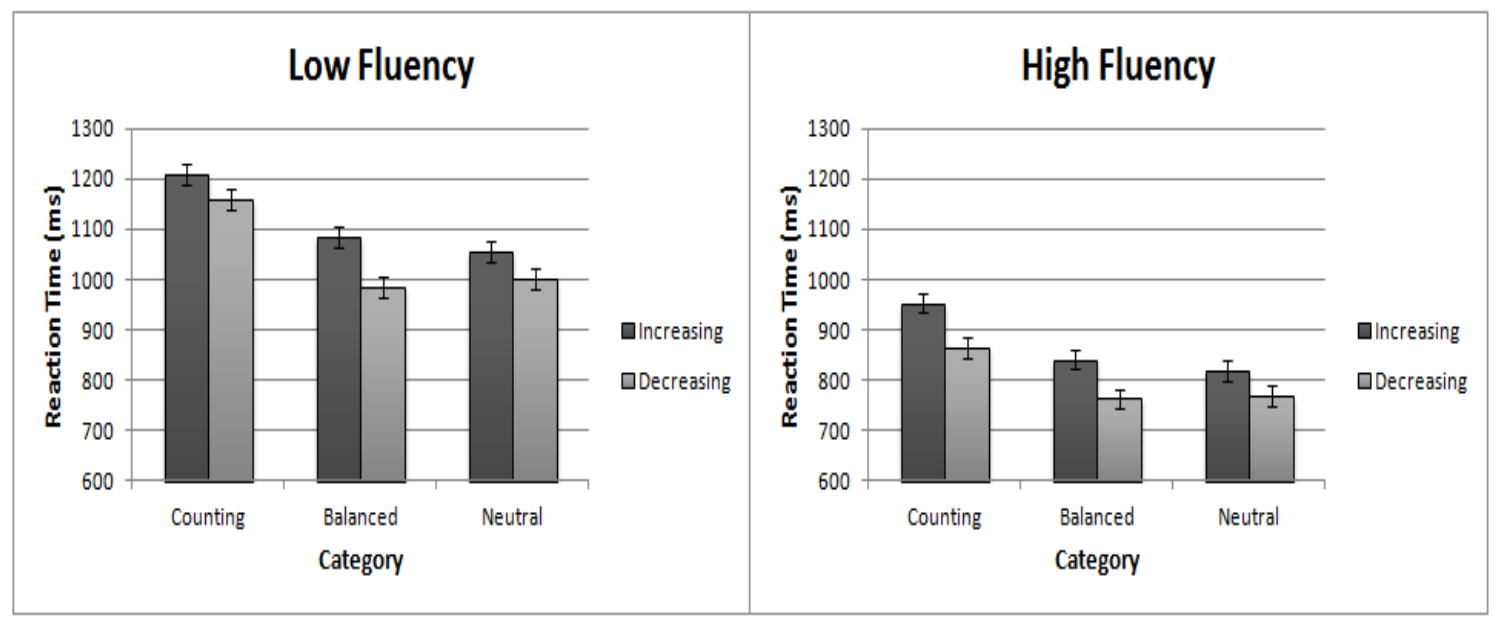

Figure 4. Unordered "increasing" and "decreasing" mean response times for high and low fluency participants across the counting, balanced, and neutral categories.

Error Rates

Error rates (\%) were analyzed in a 2(fluency: low, high) x 4(category: counting, balanced, arithmetic, neutral) x 2(sequence-order: ordered, unordered) mixed ANOVA with category and sequence-order as repeated measures factors and fluency as a betweengroups factor. High-fluency participants made fewer errors than low-fluency participants $(4.4 \%$ vs. $6.3 \%), F(1,58)=6.16, p=.016, \eta_{\mathrm{p}}{ }^{2}=.096$. Errors also varied by category, $F(3,174)=32.84, p<.001, \eta_{\mathrm{p}}{ }^{2}=.361$, with counting stimuli showing the greatest number of errors $($ counting $=8.0 \%$, balanced $=3.7 \%$, arithmetic $=5.6 \%$, neutral $=$ $4.1 \%)$. Errors were also found to vary by sequence-order, $F(1,58)=37.11, p<.001, \eta_{\mathrm{p}}{ }^{2}$ $=.390$, with unordered responses showing higher error rates than ordered responses $(7.0 \%$ vs. $3.7 \%)$. 
Table 6. Error rates (\%) for arithmetic fluency groups representing a median split of participants by their fluency scores.

Stimulus Category

\begin{tabular}{lccccccccc}
\cline { 3 - 8 } Sequence-order & \multicolumn{2}{l}{ Counting } & \multicolumn{2}{l}{ Balanced } & \multicolumn{2}{l}{ Arithmetic } & \multicolumn{2}{l}{ Neutral } \\
& $M$ & $S E$ & $M$ & $S E$ & $M$ & $S E$ & $M$ & $S E$ \\
\hline & & & & Low fluency & & & \\
Ordered & 3.8 & $(.8)$ & 3.8 & $(.8)$ & 4.7 & $(.7)$ & 5.0 & $(.7)$ \\
Unordered & 15.3 & $(1.4)$ & 4.5 & $(.5)$ & 8.0 & $(1.2)$ & 5.1 & $(.7)$ \\
& & & & & High fluency & & & \\
Ordered & 2.4 & $(.8)$ & 3.2 & $(.8)$ & 3.3 & $(.7)$ & 3.5 & $(.7)$ \\
Unordered & 10.5 & $(1.4)$ & 3.2 & $(.5)$ & 6.3 & $(1.2)$ & 2.9 & $(.7)$ \\
& & & & & & & & \\
\hline
\end{tabular}

Consistent with the reaction time data, there was a two-way interaction between category and sequence-order, $F(3,174)=40.40, p<.001, \eta_{\mathrm{p}}{ }^{2}=.411$, with a disproportionately greater number of errors in the unordered counting category than in any of the other categories (see Figure 5). These results supported the latency data in indicating that participants experienced significant interference when faced with stimuli that triggered a greater sense of familiarity. Although participants also made more errors on unordered arithmetic sequences than on their corresponding ordered sequences, recall that the unordered sequences for this problem category only included the more difficult "increasing" sequences. Thus, this comparison should be interpreted cautiously. No other interactions were significant, $p \mathrm{~s}>.10$. 


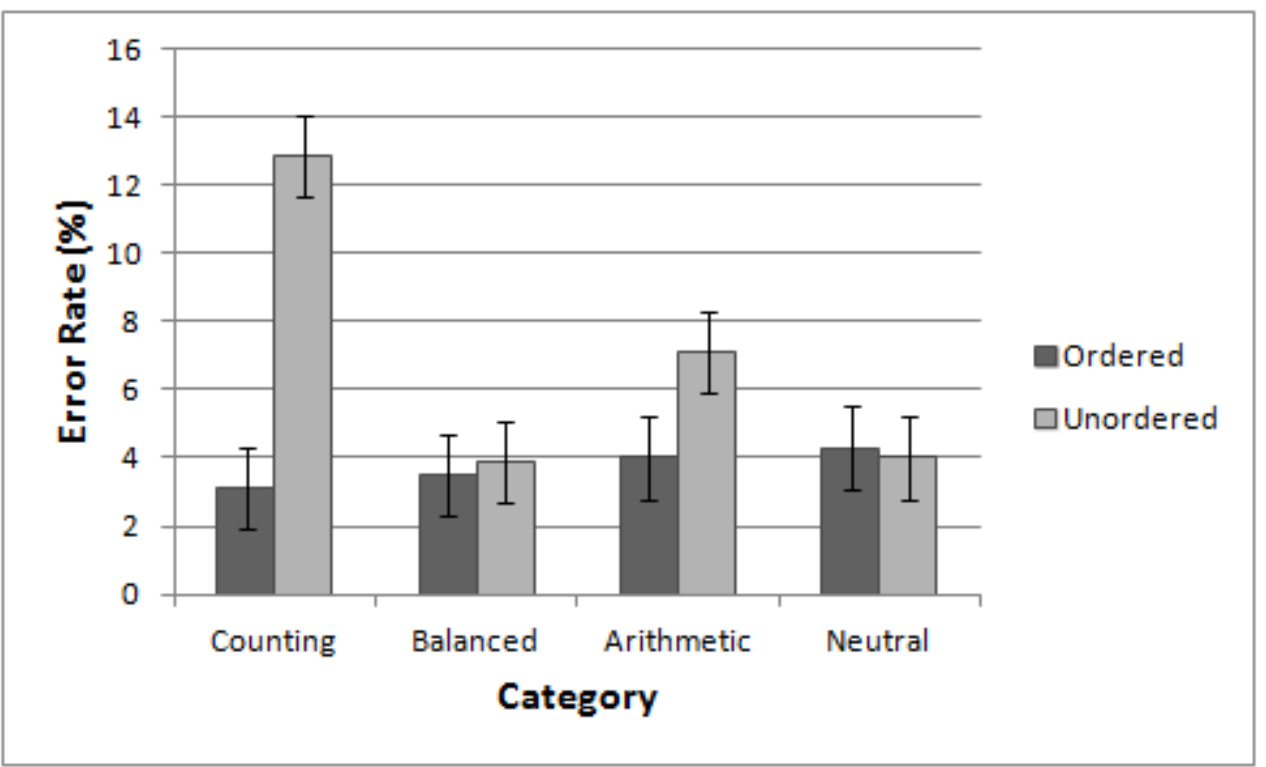

Figure 5. Error rates (\%) for ordered and unordered sequences for all categories across all participants.

Unordered sequence error rates were analysed in a 2(fluency: low, high) $\mathrm{x}$ 4(category: counting, balanced, arithmetic, neutral) x 2(sequence-order: "increasing”, “decreasing”) mixed ANOVA with category and sequence-order as repeated measures factors and fluency as a between-groups factor. Unordered sequence errors varied with fluency, $F(1,58)=8.09, p=.006, \eta_{\mathrm{p}}{ }^{2}=.122$, with high fluency participants making fewer errors than low fluency participants (5.5 vs. 8.3). Errors also varied by category, $F(2,116)=82.76, p<.001, \eta_{\mathrm{p}}{ }^{2}=.588$, with counting stimuli showing the greatest number of errors $($ counting $=12.9 \%$, balanced $=3.9 \%$, neutral $=4.0 \%)$. Errors were also found to vary by sequence-order, $F(1,58)=4.35, p=.041, \eta_{\mathrm{p}}{ }^{2}=.070$, with "increasing" sequences showing higher error rates than "decreasing" sequences (7.6\% vs. $6.2 \%)$.

No significant two-way interactions were found, $p \mathrm{~s}>.05$. However, there was a significant three-way interaction among category, sequence-order, and fluency, $F(2,116)$ $=5.11, p=.007, \eta_{\mathrm{p}}{ }^{2}=.081$ (see Figure 6). For both fluency groups, unordered “decreasing" and "increasing" sequences showed similar rates of error in the balanced 
and neutral categories. However, low fluency participants made more errors on counting “increasing" sequences (e.g., 35 4) than on counting "decreasing" sequences (e.g., 53 4; see Figure 5), suggesting that low fluency participants may have had greater difficulty than high fluency with overcoming the interference effects associated with the “increasing” counting sequences. See Table 7 for means.

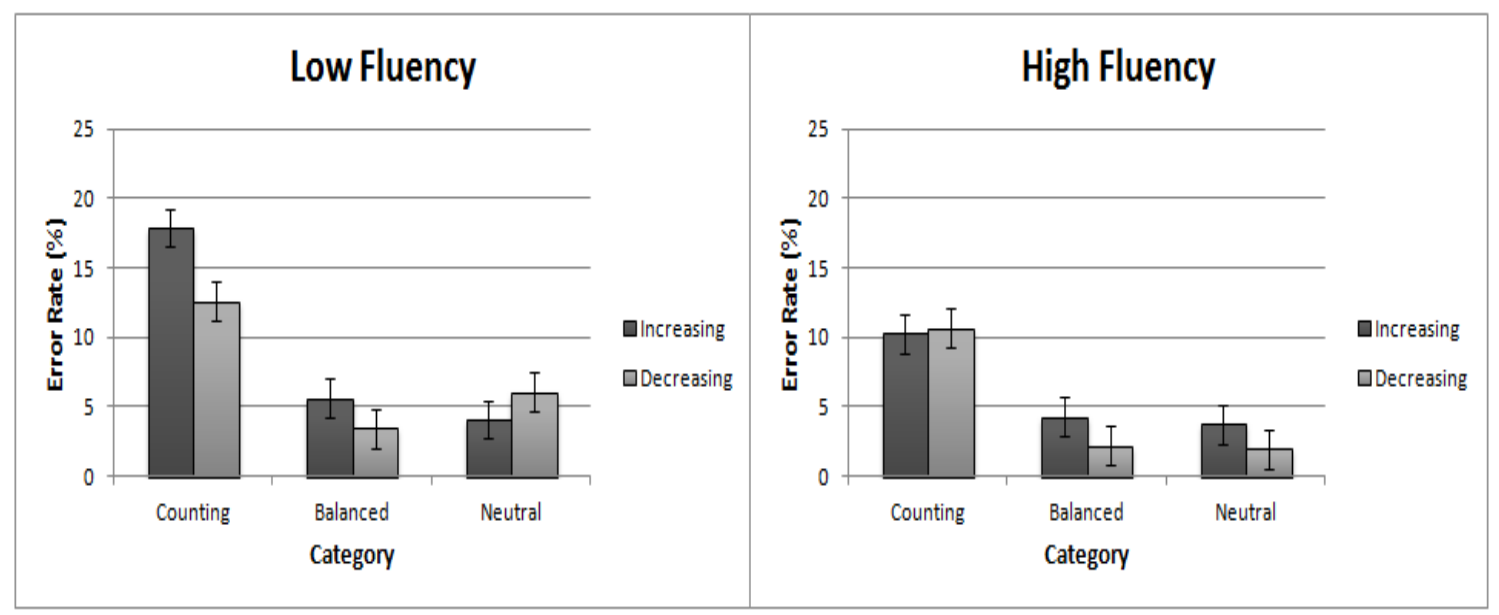

Figure 6. Error rates (\%) for unordered "increasing" and "decreasing" sequences for all categories across all participants. 
Table 7. Error rates (\%) for unordered "increasing" and "decreasing" sequences by high and low arithmetic fluency groups.

Stimulus Category

\begin{tabular}{|c|c|c|c|c|c|c|}
\hline \multirow[t]{2}{*}{ Sequence-order } & \multicolumn{2}{|c|}{ Counting } & \multicolumn{2}{|c|}{ Balanced } & \multicolumn{2}{|c|}{ Neutral } \\
\hline & $M$ & $S E$ & $M$ & $S E$ & $M$ & $S E$ \\
\hline & \multicolumn{6}{|c|}{ Low fluency } \\
\hline Increasing & 17.9 & $(2.2)$ & 5.6 & $(.8)$ & 4.1 & $(.8)$ \\
\hline \multirow[t]{2}{*}{ Decreasing } & 12.6 & $(1.3)$ & 3.5 & $(.7)$ & 6.1 & $(1.0)$ \\
\hline & \multicolumn{6}{|c|}{ High fluency } \\
\hline Increasing & 10.3 & $(.022)$ & 4.3 & $(.8)$ & 3.8 & $(.8)$ \\
\hline Decreasing & 10.7 & $(.013)$ & 2.2 & $(.7)$ & 2.0 & $(1.0)$ \\
\hline
\end{tabular}

\section{Discussion}

In the present research, I replicated the main findings reported by Lyons and Beilock (2009), specifically, that participants responded to ordered counting sequences quickly and accurately but were much slower and made more errors on the unordered counting sequences. Individuals were faster at recognizing ordered counting sequences than either balanced or neutral sequences. Lyons and Beilock (2009) suggested that these patterns occurred because participants used ordinal knowledge to determine if sequences were ordered. They assumed that counting sequences have a higher degree of ordinal association between digits, whereas balanced stimuli have somewhat weaker associations, and neutral sequences have extremely weak ordinal associations between their constituent numbers. Lyons and Beilock also proposed that the ability of those with 
high working memory to infer more complex ordinal relationships allowed them to more quickly recognize and respond to ordered balanced sequences (i.e., treated balanced sequences more like counting sequences). Conversely, those with lower working memory responded essentially the same way to either ordered or unordered balanced sequences (i.e., treated balanced sequences more like neutral sequences).

Despite their claims, the results of the current study supported an alternative view. The presence of large interference effects for counting sequences, fairly small effects for balanced (for those with high arithmetic fluency), and lack of interference for neutral sequences, indicated familiarity with the sequences in question and not a greater sense of ordinality was the determining factor in performance and drove the observed individual differences. Familiarity with specific sequences is a result of repeatedly seeing, practicing with, and internalizing these sequences. The persistence of the counting sequence in number patterns provides a strong and consistent exposure to these associations. Automaticity would make recognition of ordered sequences easier and potentially faster, but it would also make it more difficult to reject unordered sequences that resemble familiar ordered sequences. Although interference was also observed for arithmetic sequences, this result was confounded by having only included one type of unordered sequence (i.e. "increasing") for comparison, which was determined to be necessarily slower. This experimental limitation was addressed in Experiment 2.

Automaticity in sequence recognition would certainly account for why there were clear interference effects for "unordered" counting sequences, but not in the neutral sequences. Moreover, both highly- and less-skilled participants responded faster to ordered counting sequences than to ordered neutral sequences, which was not surprising 
given that, of the two equally ordered types, ordered counting sequences were much more familiar. This result supported the idea that familiarity, and not ordinality, was the source of individual differences in performance on these sequences. Both high and low fluency participants responded more slowly to unordered counting stimuli than to any other sequences, suggesting that the level of familiarity associated with counting sequences was high for both groups. Nevertheless, the high fluency participants still responded faster on both ordered and unordered counting sequences, indicating that their overall ability to process symbolic digits was superior to that of low fluency individuals.

Longer response latencies for unordered “increasing” sequences, such as 175 , and shorter latencies for "decreasing", such as 715 , supported the view that individuals employ specific strategies to efficiently process numerical sequences. The similarity of the response patterns by both high and low fluency individuals to these sequences indicated that similar strategies were being used, the most obvious being a contextdependent sequential decision rule, which stipulated that having the first two digits of a number sequence be in decreasing order violated the ascending rule (i.e. $1^{\text {st }}$ digit $<2^{\text {nd }}$ digit $<3^{\text {rd }}$ digit) and thus allowed it to be more easily rejected as an ordered sequence. Having shorter response latencies for the less familiar unordered "decreasing" balanced and neutral sequences, while also having longer response latencies for the extremely familiar unordered "decreasing" counting sequences, despite the use of a sequential decision rule, provided support for the argument that numerical sequence processing was not entirely self-terminating. Essentially, individuals examined the first two digits to determine if they were in increasing order. If they were not, those sequences could then be labelled as "unordered". However, due to the small set size of three single digits, at 
least partial processing of the third digit would still take place. Consequently, if the sequence being examined was a highly familiar sequence, like a counting sequence, then obligatory activation would occur and inhibition of this familiarity response would be required before the sequence could be rejected, resulting in longer response latencies or interference (especially for low fluency individuals). Less familiar sequences would cause less interference and could thus be rejected more quickly. Therefore, the main advantage of the rule violation was likely that it served as an added cue or confirmation that a sequence was not ordered, thus helping to speed up decision times and assist participants in overcoming interference effects, rather than reducing incoming information.

Regarding accuracy, participants showed more interference effects and made more errors on unordered counting stimuli than on balanced or neutral stimuli. Unordered arithmetic sequences also showed more errors, but again arithmetic sequences were confounded and thus could not be properly compared to the other categories. Overall, these results provided additional support for the familiarity hypothesis. Notably, high fluency participants made fewer errors than low fluency individuals on unordered "increasing" counting sequences, indicating that high fluency individuals are more capable of compensating for the presence of interference effects.

In summary, unordered counting sequences across all participants elicited slower responses than unordered neutral sequences, suggesting that something other than order information is the determining factor in sequence recognition. In contrast, balanced sequences such as 258 only elicited interference for individuals with good arithmetic skills (which replicates the results of LeFevre \& Bisanz, 1986). The participants who had 
better arithmetic skills responded more quickly to ordered than unordered balanced sequences, suggesting that their store of memorized or familiar patterns was greater than that of low-skilled individuals. High arithmetic fluency is developed in large part through practice, thus those with high arithmetic fluency are more likely to have had significantly more exposure to balanced sequences and can thus more quickly recognize them as ordered, while also having difficulty rejecting an unordered response that resembles a familiar sequence. In addition, all participants were capable of using strategies to their advantage. However, although strategies could help in the effective processing of numerical sequences, interference effects tended to be highly resilient and very difficult to overcome, regardless of fluency.

The goal of Experiment 2 was to extend the results and address the limitations of Experiment 1. One important limitation of Experiment 1 involved the unordered stimuli for arithmetic sequences. When comparing "increasing" and "decreasing" unordered sequences, it was not possible to include arithmetic sequences in the analysis, as there were no unordered "decreasing” sequences for comparison. Therefore, it was not possible to determine with certainty whether or not the interference effect observed for unordered arithmetic sequences was in fact a true interference effect or simply an artefact of having only "increasing” unordered sequences (which are necessarily slower). Resolving this confound was important, because if the interference effect observed for unordered arithmetic sequences was due to the absence of unordered "decreasing" stimuli in this category, then only counting sequences were sufficiently familiar to cause interference, whereas balanced, arithmetic, and neutral sequences were all fairly equivalent, with only small or negligible effects. This interpretation appeared particularly plausible when 
examining Figure 7, where ordered and both versions of unordered stimuli were combined for ease of interpretation.

As shown in Figure 7, low fluency individuals' response times were slower on unordered "increasing" sequences than on ordered sequences and faster on unordered “decreasing" sequences for both the balanced and neutral categories and thus, the responses times for the two types of unordered sequences averaged out to be the same as for the ordered sequences. For these individuals, the slower response times on “increasing" sequences may simply be a consequence of the general tendency for decisions involving rejection to be more difficult than confirmatory responses, requiring inhibition of a preliminary "yes" or ordered response before a decision can be made (Forster \& Hector, 2002). Unordered “decreasing” sequences would normally display a similar slowdown, except that the sequential rejection rule participants were using would allow them to more easily reject these sequences.

In contrast, for high fluency participants, response times on ordered balanced sequences were as fast as on unordered "decreasing” sequences, in support of the view that those ordered sequences were to some extent more familiar to them than to the low fluency group. Thus, the interference effect observed in the overall analysis of unordered balanced sequences reflected the slower latencies displayed by the highly-skilled group for "increasing" balanced sequences. Moreover, the unordered "decreasing" response latencies for both balanced and neutral sequences were basically identical. For there to be evidence of a true overall or general interference effect for balanced sequences, unordered "decreasing" balanced sequences would have to be significantly slower than their neutral counterparts, as exemplified by "decreasing” counting sequences. 
Examination of unordered arithmetic "increasing" and "decreasing" response patterns was thus important in order to determine whether or not their observed interference effect was genuine and whether arithmetic sequences could be used to support familiarity versus ordinality.

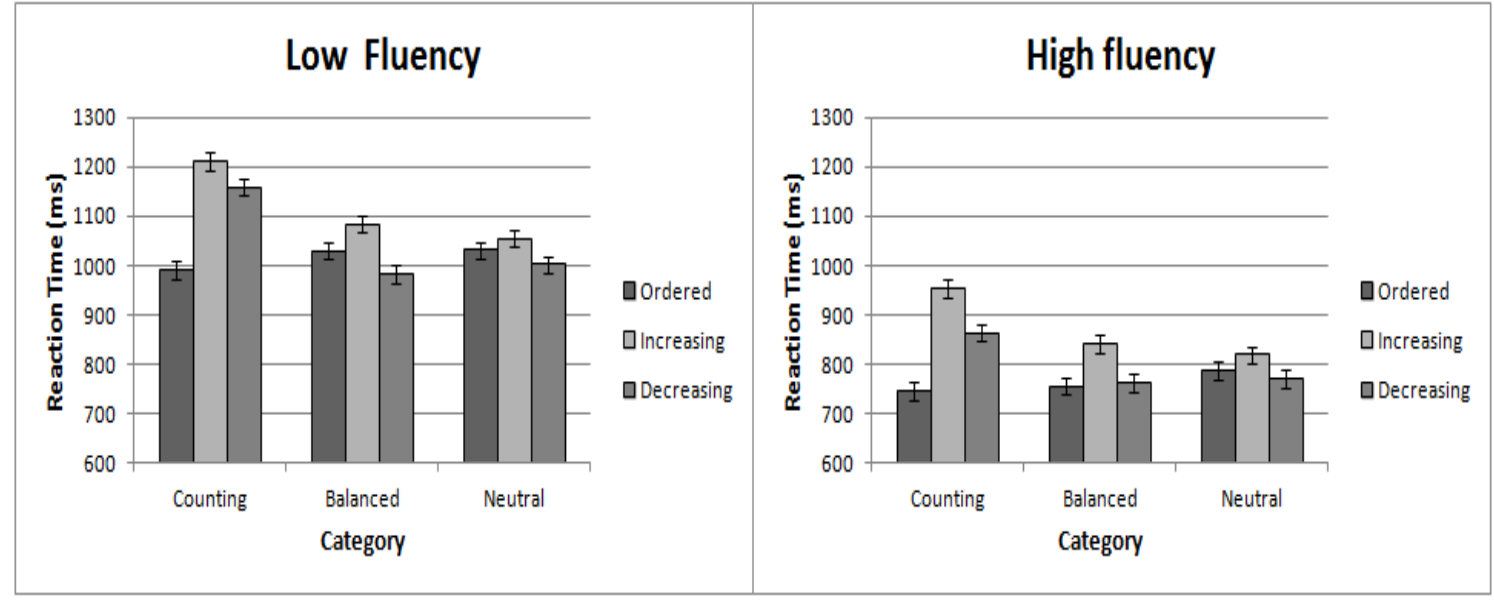

Figure 7. Unordered "increasing", "decreasing", and ordered mean response times for all categories across all participants.

Experiment 2 also extended Experiment 1 in that descending sequences were included in the list of stimuli used in the ordering task. The inclusion of descending sequences permitted further testing of the claim that familiarity is the primary factor in sequence recognition. If participants show similar reaction times for ascending and descending ordered sequences, then ordinality would be supported (because the ordinal relationship is the same between the digits - 321 vs. 123 ). If participants were faster to process ascending sequences than descending sequences, then familiarity would be supported, because although forward counting is practiced repeatedly from an early age, counting backwards is practiced significantly less and is considerably more difficult, resulting in high numbers of systematic errors (Nairne \& Healy, 1983). For this reason, counting backwards is frequently used in sobriety testing and as a cognitive load task in 
research on attention and short-term memory (e.g., Andersson, Hagman \& Talianzadeh, 2002; Lewis \& Linder, 1997; Simoneau, Teasdale \& Bourdin, 1999; Sweller, 1988). 


\section{EXPERIMENT 2}

Although the design of Experiment 2 was very similar to the previous experiment, there were a few important modifications. One of the changes involved the ordering task itself. The new ordering task included the same item categories as in Experiment 1 (i.e., counting, balanced, arithmetic, and neutral), but implemented some new stimuli and order classifications. More specifically, the original ordering task consisted of only ascending ordered sequences (i.e. $1^{\text {st }}$ digit $<2^{\text {nd }}$ digit $<3^{\text {rd }}$ digit) with one or two of their possible corresponding unordered sequences. In contrast, Experiment 2 included descending (i.e. $1^{\text {st }}$ digit $>2^{\text {nd }}$ digit $>3^{\text {rd }}$ digit) ordered sequences along with all four possible unordered arrangements (two "increasing" and two "decreasing") of each sequence. The purpose of descending sequences was to represent numerical sequences that were less familiar, but equally ordinal, as their ordered ascending counterparts. If response patterns for ascending and descending ordered sequences were identical, then ordinality would be supported as the primary factor in sequence recognition. However, if descending sequences showed greater response latencies and higher rates of error than ascending, then familiarity would be supported.

Other than the stimulus modifications, there was also an important design change. Rather than dividing stimuli into four equivalent blocks that only served to break up the task into smaller chunks, stimuli in Experiment 2 were separated into three distinct blocks, or experimental conditions, classified by the type of ordered sequences they contained (i.e. Ascending condition: comprising ascending ordered, plus "increasing" and “decreasing” unordered sequences; Descending condition: comprising descending ordered, plus "increasing" and "decreasing" unordered sequences; Mixed condition: order 
condition comprising ordered ascending and descending sequences, plus unordered "increasing" and "decreasing" sequences). The reason for separating stimuli into orderspecific conditions, or blocks, was to determine how strategy selection could be influencing response patterns. For example, in Experiment 1 participants always responded more quickly to unordered "decreasing" sequences than to their "increasing" versions. It was hypothesized that this pattern was due to participants being able to more easily exclude "decreasing" sequences after observing that the second digit was smaller than the first (i.e. $1^{\text {st }}$ digit $>2^{\text {nd }}$ digit, thus could not possibly be an ascending sequence), whereas an "increasing" unordered sequence required full examination of all three digits before a decision could be made. If this hypothesis was true, then a similar, but reversed, pattern would be expected for an experimental condition containing only descending and unordered sequences (i.e., Descending condition). More specifically, this strategy would favour faster rejection of unordered "increasing" sequences (i.e. $1^{\text {st }}$ digit $<2$, , therefore could not possibly be descending). The Mixed condition included all possible sequenceorders, thus removing the utility of the previous strategy. By invalidating obvious strategic short-cuts, it is expected that individuals will be forced to fully examine each sequence and that any observed response time or accuracy advantages can be attributed to the stimuli themselves and not the contextual circumstances.

In order to be able to examine and compare the response patterns for the different sequence-orders (i.e. ordered: ascending, descending; unordered: “increasing”, "decreasing"), rather than only compare ordered sequences to unordered sequences (as in Experiment 1), analyses focused on comparing all four sequence-orders in greater detail.

\section{Predictions}


Results for the Ascending condition (i.e., experimental condition containing only ascending and unordered sequences) were expected to replicate the findings of Experiment 1. Specifically, counting ascending sequences would show the fastest responses and the slowest "unordered" responses of all four categories, due to strong familiarity-induced interference effects. In contrast, response patterns for balanced, arithmetic, and neutral sequences were expected to be quite similar, with no categoryspecific general interference effects (i.e., interference effects for both unordered types) being found for balanced and arithmetic sequences, even for the high fluency group. For arithmetic sequences, there was the potential for seeing an interference effect (given that the unordered "increasing" arithmetic sequences were significantly slower than “increasing” neutral sequences in Experiment 1), but even so it would be fairly small. Based on the assumption held in Experiment 1 that participants would use a sequential decision strategy, it was predicted that participants would respond that a sequence was unordered much more quickly when the expected ascending pattern was violated. Thus, responses to items such as 312 would be faster than to 132 , because the descending pattern in the first two digits would permit a relatively fast rejection of the sequence as ordered. Therefore, unordered "increasing” sequences should have longer response latencies than their corresponding "decreasing" sequences.

Results for the Descending condition (i.e., experimental condition containing only descending and unordered sequences), were expected to closely reflect those for the Ascending condition, except that the sequential rejection strategy would now favor faster responding for unordered "increasing" sequences, instead of their "decreasing" versions 
(i.e. 132 would be faster than 312 , as the first two digits in the former violate the expected descending pattern).

The Mixed condition compared all four sequence-orders (i.e. ordered: ascending, descending; unordered: “increasing”, “decreasing”). For both fluency groups, ascending sequences were expected to be faster and show fewer errors than their descending versions (especially for counting). However, low fluency individuals were expected to also respond to descending sequences more like unordered sequences, due to being relatively unfamiliar with this type of sequence. With no obvious rejection rule or sequential decision strategy applicable to the Mixed order condition, the two types of unordered sequences were expected to show fairly equivalent response times and error rates.

To summarize, there were four main hypotheses for Experiment 2:

1) Participants should respond fastest to counting ordered sequences (most familiar) and slowest to their unordered counterparts (i.e., interference effects);

2) For both fluency groups, ascending sequences were predicted to be faster and more accurate than their descending versions (especially for counting);

3) The sequential decision strategy was expected to favor faster responding to unordered "decreasing" sequences, than to their "increasing" counterparts in the Ascending condition, whereas a reversal of this pattern was predicted for the Descending condition. The inapplicability of this strategy in the Mixed condition was predicted to result in more equivalent response patterns for both types of unordered sequence.

4) Both fluency groups were expected to show fairly equivalent response patterns for balanced, arithmetic and neutral sequences. 


\section{Method}

\section{Participants}

Seventy-eight (47 female and 31 male) were recruited for Experiment 2 (age: 1742 years; $M=23$ years; $S D=5.75)$. The data from 6 additional participants were excluded from the study. Two of the participants were excluded because they had corrupted/missing data, one had atypical data scores (e.g. exceedingly fast reaction times, but extremely low fluency score), and three far exceeded the upper age limit of 42 years and were thus excluded to avoid potential age effects. All participants completed two tasks: an arithmetic fluency measure and a sequence ordering task.

\section{Materials}

The fluency measure used in Experiment 2 was the same paper-and-pencil test of complex arithmetic that was used in Experiment 1 (see Appendix B). The total sum of correct addition, subtraction, and multiplication problems yielded a participant's arithmetic fluency score.

The new ordering task in Experiment 2 was similar to the version used in the first experiment, but with some important modifications. Participants were required to indicate whether 3-digit sequences were in order from left to right by pressing one of two buttons on the same RB-730 response pad used in Experiment 1. There were a total of seven buttons on the response box: three coloured (the 2, 4, and 6 response keys) and the rest white (not used during the experiment). Buttons 2 and 6 were the "ordered" and "not ordered" response buttons, respectively, and button 4 was for skipping menus.

\section{Design}


The four previously used stimulus categories (counting, balanced, arithmetic, neutral) were again used in the new ordering task. However, instead of three sequenceorders (i.e. ordered: ascending; sequence-order: “increasing”, “decreasing”), four were now possible: ascending, descending, "increasing" (unordered sequence starting with an increasing digit pair), and "decreasing" (unordered sequence starting with a decreasing digit pair). For every 3-digit numerical sequence used in Experiment 2 (e.g. 12 3), there were six possible orders of each combination of three digits: one ascending, one descending, and four unordered (two "increasing" and two "decreasing”). All six of these order arrangements were used in Experiment 2. In order to avoid the creation of an expectation bias towards unordered sequences (i.e., more likely to expect unordered sequences since proportionally there are two unordered for every ordered sequence), presentations of ordered sequences were repeated four times per participant, whereas unordered sequences were only repeated twice (see Table 5).

Table 8. Examples of the Six Possible Orders of Digits for each type of sequence.

\begin{tabular}{lcccccc}
\hline & \multicolumn{2}{c}{ Ordered } & \multicolumn{2}{c}{ Unordered } \\
\hline & Asc. & Desc. & "Incr." & "Incr." & "Decr." & "Decr." \\
\hline Counting & $\mathbf{3 4 5}$ & 543 & $\mathbf{3 5 4}$ & 453 & $\mathbf{5 3 4}$ & 435 \\
Arithmetic & $\mathbf{1 3 4}$ & $431^{1}$ & $\mathbf{1 4 3}$ & 341 & $413^{1}$ & $314^{1}$ \\
Balanced & $\mathbf{2 5 \mathbf { 5 }}$ & 852 & $\mathbf{2 8 5}$ & 582 & 825 & 528 \\
Neutral & $\mathbf{1 4 6}$ & 641 & 164 & 461 & 641 & $\mathbf{4 1 6}$ \\
\hline
\end{tabular}

Note. Sequences types in bold were used in Experiment 1.

${ }^{1}$ These sequences have an arithmetic relation among the digits (either addition or subtraction). 
The list of stimuli for Experiment 2 consisted of 252 different 3-digit sequences (see Table A.2 in Appendix A). Of these 252 sequences, 42 were counting sequences, 54 were balanced sequences, 72 were arithmetic, and 84 were neutral. Note that although all possible counting, balanced, and arithmetic sequences were used in the experiment, not all neutral sequences were included. The reason for this exclusion was due to the disproportionately greater number of possible neutral sequences. Instead a smaller quasirandom sample of neutral sequences, out of all possible neutral sequences, was selected for the experiment. The full set of stimuli is shown in Appendix A.

The ordering task itself consisted of 672 trials. There were 112 counting sequence trials (56 “ordered", 56 "unordered"), 144 balanced sequence trials (72 ordered sequences and 72 unordered), 192 arithmetic sequence trials (96 ordered, 96 unordered), and 224 neutral sequence trials (112 ordered, 112 unordered). These 672 trials were separated into three lists of 224 trials and arranged into 3 separate blocks or experimental conditions, where they were then randomly presented to the participant. One experimental order condition was classified as the Ascending condition and had only ascending ordered sequences, along with two of each of their unordered sequence counterparts (one “increasing”, one "decreasing”). A second order condition was classified as the Descending condition, containing only descending ordered sequences with two of their corresponding unordered counterparts (one "increasing", one "decreasing"). The third order condition was classified as the Mixed condition, containing both the ascending and descending versions of each sequence (e.g., 123 and 321), as well as two corresponding unordered sequences for each ordered sequence. 
Assignment of stimulus list to order condition (Ascending, Descending, Mixed) was counterbalanced across participants in order to avoid potential confounds or biases that could result from having certain unordered sequences always associated with the same order condition (e.g., 314 always appearing in the Ascending condition only). The order in which participants did each experimental order condition of the three was also counterbalanced.

\section{Procedure}

Participants were asked to complete two tasks related to the current study in a single testing session: the fluency measure (employed in Experiment 1) and the new number sequence ordering task. For the sequence ordering task, participants were asked to indicate whether 3-digit sequences were in order from left to right and informed that this applied to both ascending and descending sequences (i.e., participants had to be able to recognize both types as being "ordered"). Button pressing was counterbalanced so that half of the participants would respond that a sequence was "ordered" by pressing the left green button (button 2) and "unordered" by pressing the right blue button (button 6), whereas for the other half of participants the locations of the buttons were reversed (i.e., the response box was rotated so that the green button, button 2, was on the right side of the response box and the blue button, button 6, was on the left). Again, participants had to keep their hands positioned with a finger on each response button, so that they could immediately press the appropriate button once a decision had been made.

In order to help participants become more familiar with the task, they all received the same 16 practice trials, with accompanying feedback regarding the accuracy of their responses. The 16 practice trials involved four counting (two ordered and two unordered), 
four balanced (two ordered and two unordered), four arithmetic (two ordered and two unordered), and four neutral (two ordered and two unordered) sequences. After the practice trials there was a break, allowing participants to have time to ask questions and giving the experimenter the opportunity to ensure their understanding of the task. Once ready, participants moved on to the experimental trials, where no additional feedback about the individual trials was provided.

Similar to Experiment 1, each trial consisted of three single-digit Arabic numerals being presented on a computer screen for a maximum of $1500 \mathrm{~ms}$. If participants had not responded by this time, the stimuli would vanish, leaving only a black background until they did respond. Whenever a response was detected, three vertical white bars appeared at the center of the screen for a maximum duration of $1500 \mathrm{~ms}$. After $1500 \mathrm{~ms}$, the bars vanished and a new 3-digit sequence appeared, thus commencing a new trial. The vertical bars had three purposes: 1) they indicated to the participant that the computer had received their answer, 2) acted as an inter-stimulus interval (ISI), during which participants could mentally ready themselves for the next trial, and 3) acted as a fixation point at which participants could focus their gaze. If participants wished to continue on to the next stimulus before the full $1500 \mathrm{~ms}$ duration of the ISI, they could do so by pressing any key on the response box. An example trial is shown in Figure 8. This procedure differed slightly from the one used Experiment 1, where the intervening ISI stimulus (three white bars) was always shown for a full $1500 \mathrm{~ms}$. Participants had found this pause before the next stimulus presentation to be too long. Allowing participants to shorten the duration of the ISI (though a minimum ISI of $250 \mathrm{~ms}$ was always retained in order to maintain some control and consistency between participants), was intended to 
reduce boredom and enable participants to more easily maintain focus and concentration throughout the task. This task modification was expected to have only reduced boredom and deter loss of concentration and not to have biased or affected participant response patterns.

1 Trial

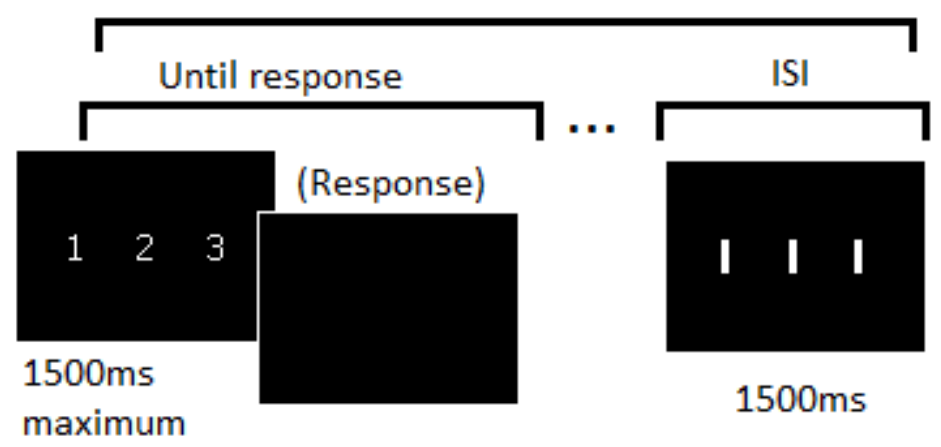

Figure 8. Trial format for ordering task in Experiment 2. Stimuli were presented for a maximum of $1500 \mathrm{~ms}$. If participants responded before this time limit, the sequence immediately moved to the inter-stimulus-interval (ISI); otherwise, stimuli disappeared after $1500 \mathrm{~ms}$, leaving a blank screen, which remained until subjects responded. The ISI was a minimum of $250 \mathrm{~ms} \mathrm{~ms}$ and a maximum of $1500 \mathrm{~ms}$. Participants were able to press a button after the first $250 \mathrm{~ms}$ to more quickly advance to the next trial. 


\section{Results}

Data screening was conducted prior to data analyses. Descriptive statistics, histograms, and z-scores were obtained for each participant's reaction time data in order to check for the presence of univariate outliers. Reaction time data is naturally skewed, therefore high standardized scores were expected and often had to be visually examined via histogram (with normal curve superimposed) in order to determine if response times were obviously different from the rest. Standardized scores greater than 5SDs in particular were scrutinized in more detail via histogram and excluded, unless they clearly fit with the data trend observed in their histograms. However, given that median response times were used for all ANOVA analyses, which are less affected by outliers than mean response times, univariate outliers were not a major concern.

\section{Arithmetic Fluency}

Individual differences in arithmetic fluency were determined by the total score (number correct) obtained on the arithmetic fluency task. A median split of the 78 total participants was used to assign participants with the highest 39 and lowest 39 fluency scores to high and low arithmetic fluency groups, respectively. See Table 2 on page 19 for descriptive statistics pertaining to both experiments.

\section{Sequence Processing}

In order to facilitate comparisons with Experiment 1 and with Lyons and Beilock (2009, 2011), separate analyses were conducted for the pure order conditions (i.e., ordered sequences were only ascending in the Ascending condition and only descending in the Descending condition) and for the Mixed condition (i.e., both ascending and descending sequences were considered ordered). Post hoc testing was done for the pure 
order and Mixed conditions using the same type of $95 \%$ confidence intervals used in Experiment 1.

Ascending and Descending order conditions. Median response times were calculated for each category per participant for correct trials only. These medians and the mean percentage of errors were analyzed in separate 2(order condition: Ascending, Descending) x 2(fluency: low, high) x 4(category: counting, balanced, arithmetic, neutral) x 3(sequence-order: ordered, increasing, decreasing) mixed ANOVAs, with order condition, sequence-order, and category as repeated measures factors and fluency as a between-groups factor (see Table 9 for ANOVA results). Post hoc testing was also conducted.

As shown in Table 9, high-fluency participants responded faster than low-fluency participants (843 vs. $1100 \mathrm{~ms}$ ) and made fewer errors (4.0\% vs. 6.7\%). Response latencies also varied by category, such that counting sequences were slower and less accurate (1043 ms; $8.6 \%$ error) than the three other categories: balanced $(950 \mathrm{~ms} ; 4.0 \%$ error), arithmetic (940 ms; 4.6\% error), and neutral sequences (954 ms; 4.3\% error). Response times also varied by sequence-order, with ordered sequences displaying faster response times than either unordered "increasing" or "decreasing" sequences (ordered= $901 \mathrm{~ms}$, increasing $=1016 \mathrm{~ms}$, decreasing $=999 \mathrm{~ms})$ and fewer errors $($ ordered $=3.9 \%$, increasing $=6.6 \%$, decreasing $=5.6 \%$ ). 
Table 9. ANOVAs (response times and errors) for Ascending and Descending conditions

\begin{tabular}{|c|c|c|c|c|c|c|c|}
\hline \multirow[b]{2}{*}{ Source } & \multirow[b]{2}{*}{$d f$} & \multicolumn{3}{|c|}{ Response Time } & \multicolumn{3}{|c|}{ Percent Error } \\
\hline & & $F$ & $p$ & $\eta_{p}^{2}$ & $F$ & $p$ & $\eta_{p}^{2}$ \\
\hline Fluency & 1,76 & $31.95 * *$ & $<.001$ & .960 & $11.55 * *$ & .001 & .699 \\
\hline Order Condition & 1,76 & .24 & .624 & .003 & 1.90 & .172 & .024 \\
\hline Category & 3,228 & $74.65 * *$ & $<.001$ & .496 & $56.51 * *$ & $<.001$ & .426 \\
\hline Sequence-Order & 2,152 & $83.47 * *$ & $<.001$ & .523 & $19.45 * *$ & $<.001$ & .204 \\
\hline Fluency x Order & 1,76 & 1.24 & .269 & .016 & 1.38 & .244 & .018 \\
\hline \multicolumn{8}{|l|}{ Condition } \\
\hline Fluency x Category & 3,228 & 2.23 & .086 & .028 & 1.12 & .343 & .014 \\
\hline Fluency x Sequence- & 2,152 & 1.75 & .178 & .022 & 1.79 & .170 & .023 \\
\hline \multicolumn{8}{|l|}{ Order } \\
\hline Order Condition $\mathrm{x}$ & 3,228 & $4.34 * *$ & .005 & .054 & .59 & .625 & .008 \\
\hline \multicolumn{8}{|l|}{ Category } \\
\hline Order Condition $\mathrm{x}$ & 2,152 & $35.24 * *$ & $<.001$ & .317 & $25.51 * *$ & $<.001$ & .251 \\
\hline \multicolumn{8}{|l|}{ Sequence-Order } \\
\hline Category x Sequence- & 6,456 & $49.59 * *$ & $<.001$ & .395 & $29.71 * *$ & $<.001$ & .281 \\
\hline \multicolumn{8}{|l|}{ Order } \\
\hline Fluency x Order & 3,228 & .94 & .423 & .012 & .30 & .829 & .004 \\
\hline \multicolumn{8}{|l|}{ Condition x Category } \\
\hline Fluency x Order & 2,152 & $2.57 \dagger$ & .080 & .033 & 1.48 & .230 & .019 \\
\hline \multicolumn{8}{|l|}{ Condition x Sequence- } \\
\hline \multicolumn{8}{|l|}{ Order } \\
\hline Fluency x Category x & 6,456 & 1.28 & .267 & .017 & 1.44 & .200 & .019 \\
\hline \multicolumn{8}{|l|}{ Sequence-Order } \\
\hline Order Condition $\mathrm{x}$ & 6,456 & $2.22 *$ & .040 & .028 & $7.54 * *$ & $<.001$ & .090 \\
\hline \multicolumn{8}{|l|}{ Category x Sequence- } \\
\hline \multicolumn{8}{|l|}{ Order } \\
\hline Fluency x Order & 6,456 & .68 & .663 & .009 & .36 & .904 & .005 \\
\hline \multicolumn{8}{|l|}{ Condition $\mathrm{x}$ Category $\mathrm{x}$} \\
\hline Sequence-Order & & & & & & & \\
\hline
\end{tabular}


$\dagger p<.10 ; * p<.05 ; * * \mathrm{p}<.01$

A significant two-way interaction was found between order condition and category, such that counting latencies were significantly longer for sequences in the Descending condition than for sequences in the Ascending condition (see Figure 9). Latencies for the remaining three categories were not different between order conditions.

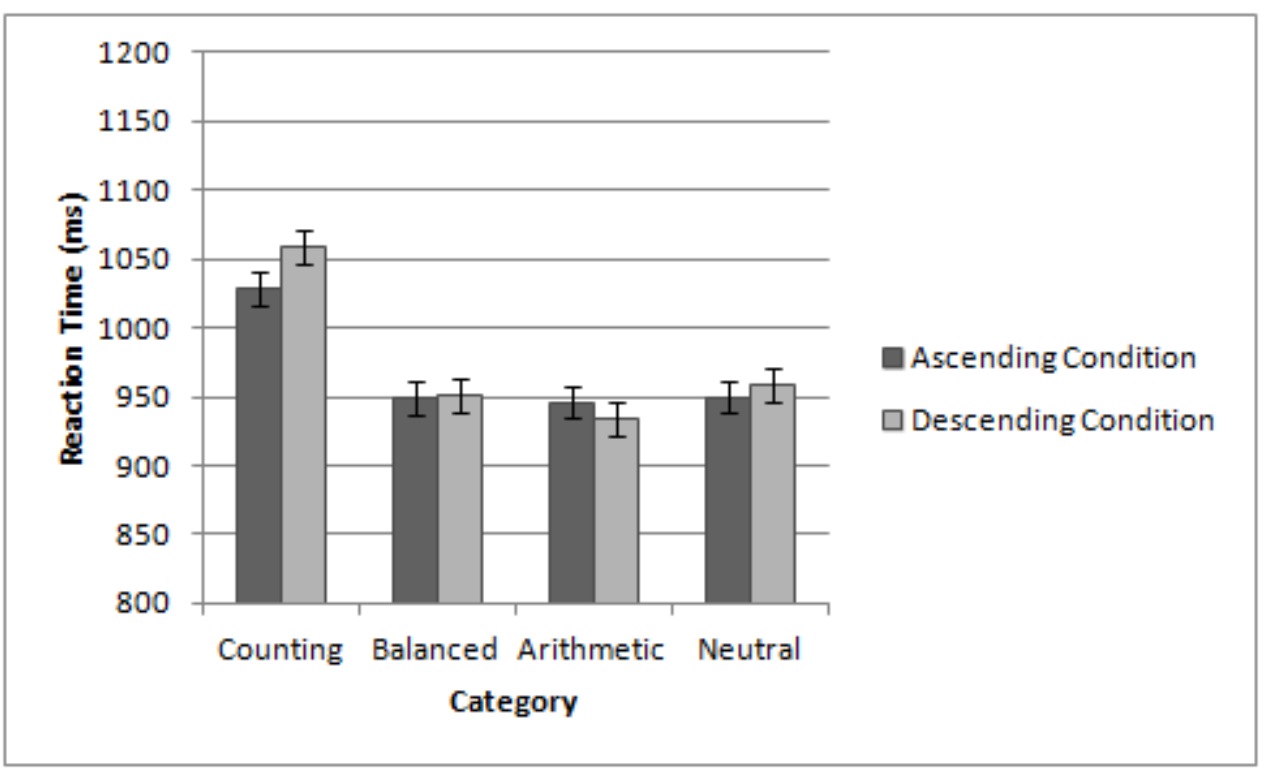

Figure 9. Experiment 2: Counting, balanced, arithmetic, and neutral mean response times compared across Ascending and Descending order conditions.

As shown in Figure 10, there was a two-way interaction between order condition and order-type. For ordered sequences, ascending items (e.g., 25 9) were faster than descending items (e.g., 95 2), supporting the hypothesis that ascending sequences are more familiar to participants than descending sequences. However, for the unordered sequences, there was a cross-over pattern: unordered "increasing" sequences were slower and showed more errors than "decreasing" sequences in the Ascending condition, whereas unordered "decreasing" sequences were slower and more prone to error than “increasing" sequences in the Descending condition. This pattern supports the hypothesis 
that participants were switching strategies and using reversed versions of the same sequential decision strategy between order conditions.

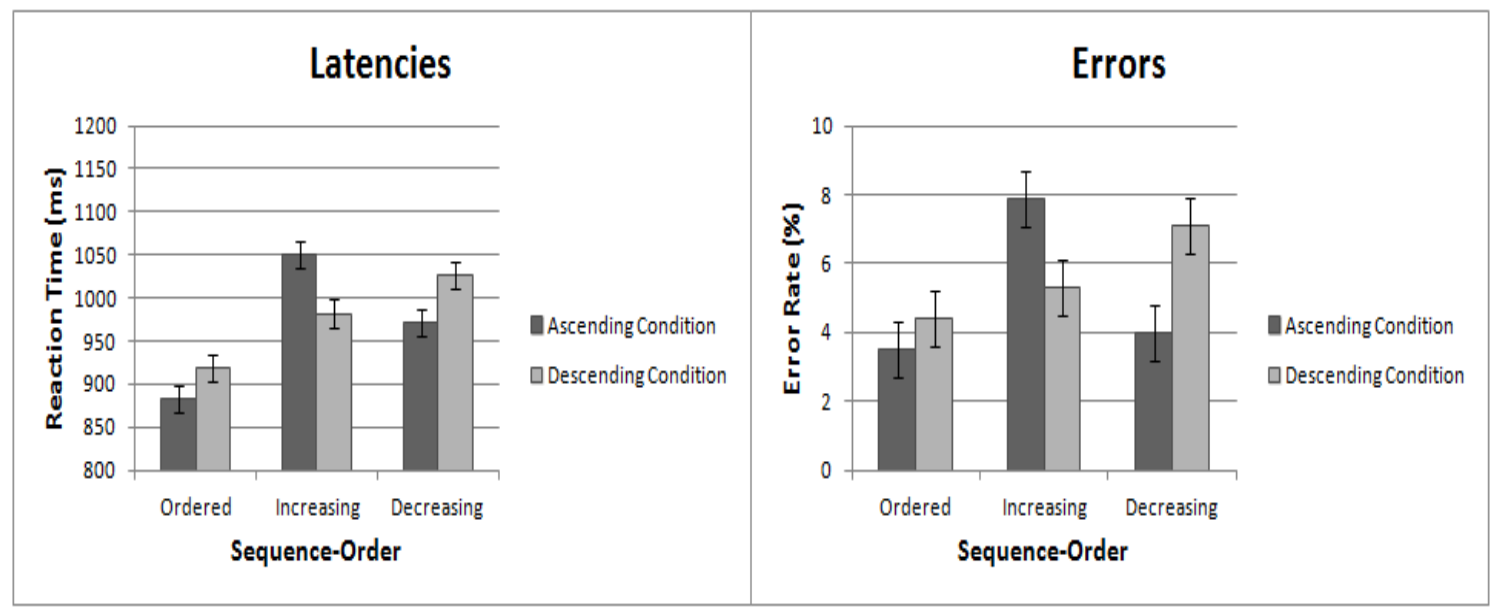

Figure 10. Experiment 2: Ordered, "increasing", and "decreasing" mean response times and error rates compared across order conditions (Ascending, Descending).

The two-way interaction between category and sequence-order is shown in

Figure 11. Participants were slowest and made the most errors on the unordered counting sequences. Unordered "increasing" and "decreasing" response latencies and number of errors were found to be quite similar across the counting, balanced and neutral categories; however, arithmetic unordered “decreasing” sequences were faster to reject and showed fewer errors than their corresponding "increasing” sequences. In contrast, arithmetic unordered "increasing” sequences showed the highest number of errors after counting unordered sequences. This interaction is qualified, however, by the three-way interaction of category and sequence-order with order condition, as shown in Figure 12. 


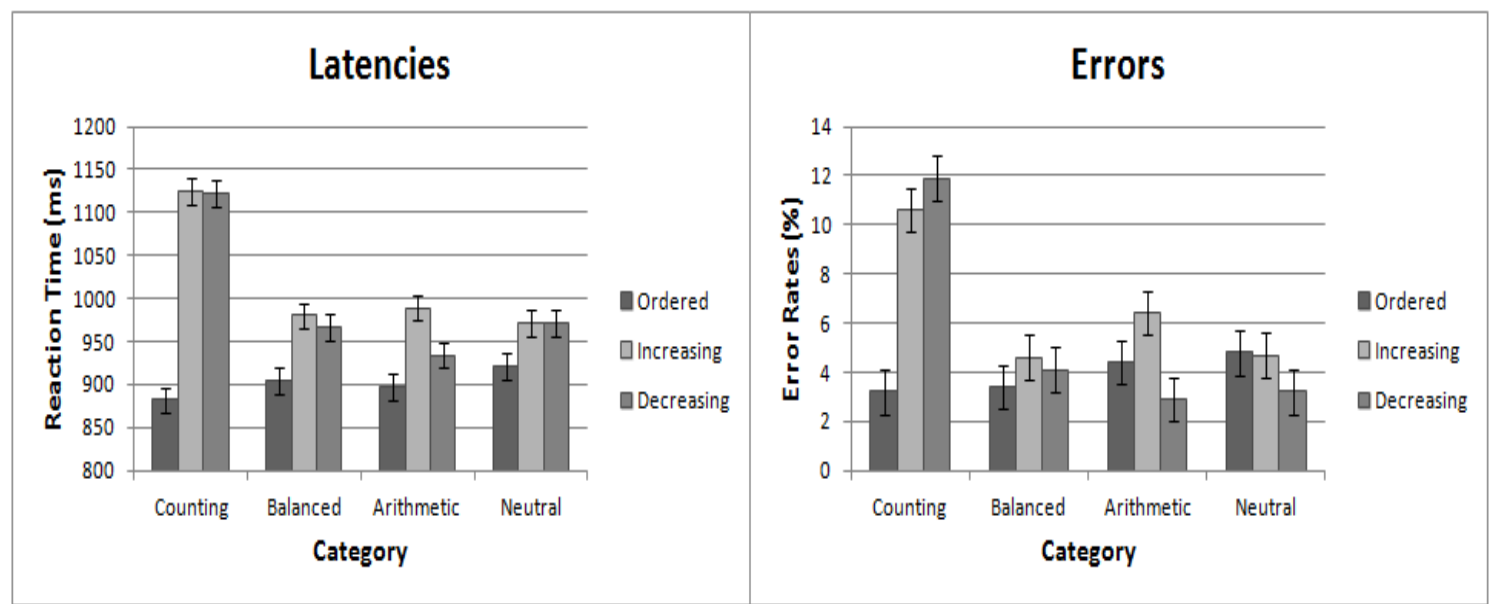

Figure 11. Experiment 2: Ordered, "increasing", and "decreasing" mean response times and error rates compared across all categories.

The three-way interaction among order condition (Ascending, Descending), category, and sequence-order is shown in Figure 12. As seen in Figure 12, ordered counting sequences (e.g., 34 5) in the Ascending condition were verified faster than those in the other three categories. In contrast, there were no differences among the ordered sequences in each category in the Descending condition. Thus, ordered counting sequences were only advantaged when the task involved verifying ascending sequences.

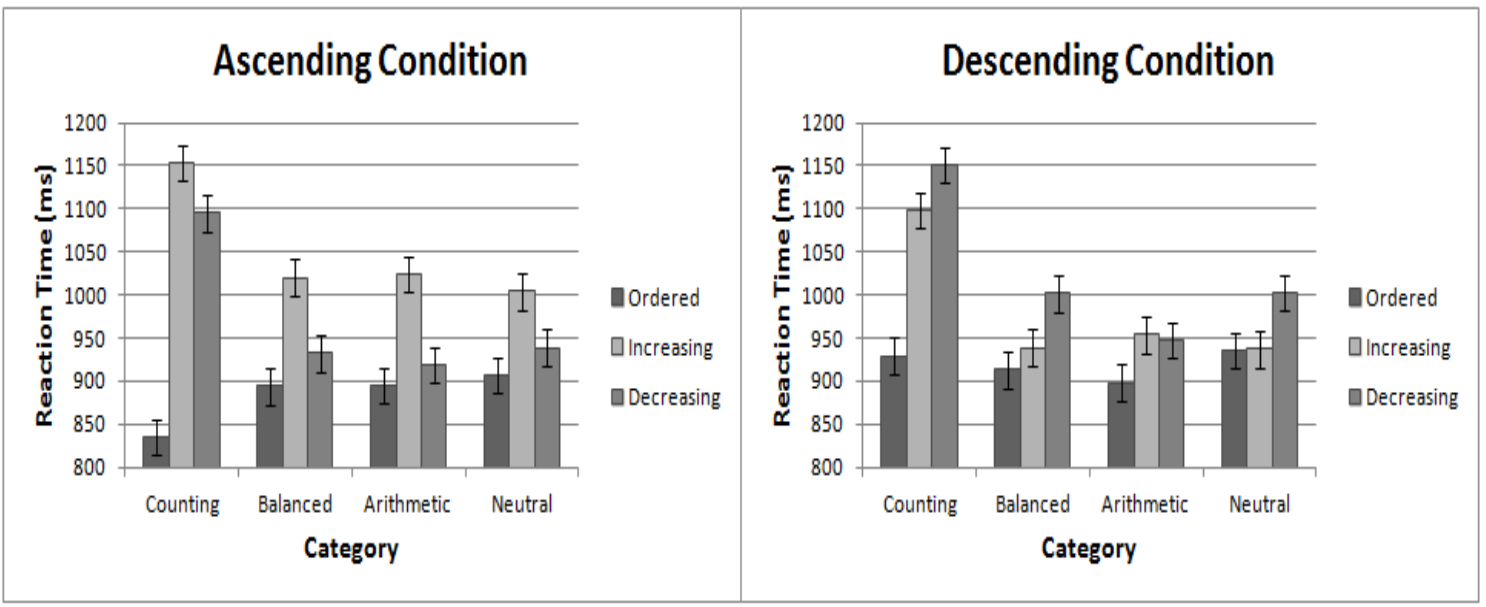

Figure 12. Experiment 2: Ordered, "increasing", and "decreasing" mean response times for all categories across order conditions (Ascending, Descending). 
For unordered sequences, the pattern in the Ascending condition supported the view that participants used a sequential decision strategy consistently across categories. For counting, arithmetic, and neutral sequences, unordered "increasing" sequences (e.g., 354 ) were always slower to reject and showed more errors than "decreasing" sequences (e.g., 53 4), whereas balanced sequences only showed the longer response times. For neutral and arithmetic sequences, there were no differences between the unordered "decreasing" and ordered versions, again supporting the view that the main influence on processing was from the sequential decision rule. Unordered counting sequences, however, were always much slower to reject and showed more errors than ordered counting sequences, reflecting obligatory interference, even in the "decreasing" condition. Balanced sequences also showed a minor reaction time difference between ordered and "decreasing" items, but the magnitude of the effect was quite small. Interestingly, arithmetic "increasing" sequences (i.e., 143 and 34 1) were much more prone to error than either of their corresponding ordered or "decreasing" sequences (i.e., 413 and 314 ). Although the response times for unordered "increasing" arithmetic sequences were fairly similar to those in the balanced and neutral categories (suggesting no additional hesitation), the fact that they showed more errors indicated that there may have been a bias towards recognizing and classifying these sequences as ordered.

In the Descending order condition, the pattern for unordered sequences was reversed, with "increasing" sequences being verified faster than "decreasing” sequences in the counting, balanced, and neutral categories. This pattern reflected that change in the task criteria (where descending sequences were the only ordered ones). Unordered "decreasing" counting sequences were also more prone to error than their "increasing" 
counterparts; however, numbers of errors were similar across sequence-orders in the remaining three categories. The arithmetic sequences, however, showed a different pattern, with slower response times (relative to the ordered sequences in this category) on “increasing" sequences (i.e., 143 and 34 1) and relatively faster latencies on the “decreasing” sequences (i.e., 413 and 3 14). This pattern was not what would be predicted if the subtraction pattern in these latter items was affecting performance.

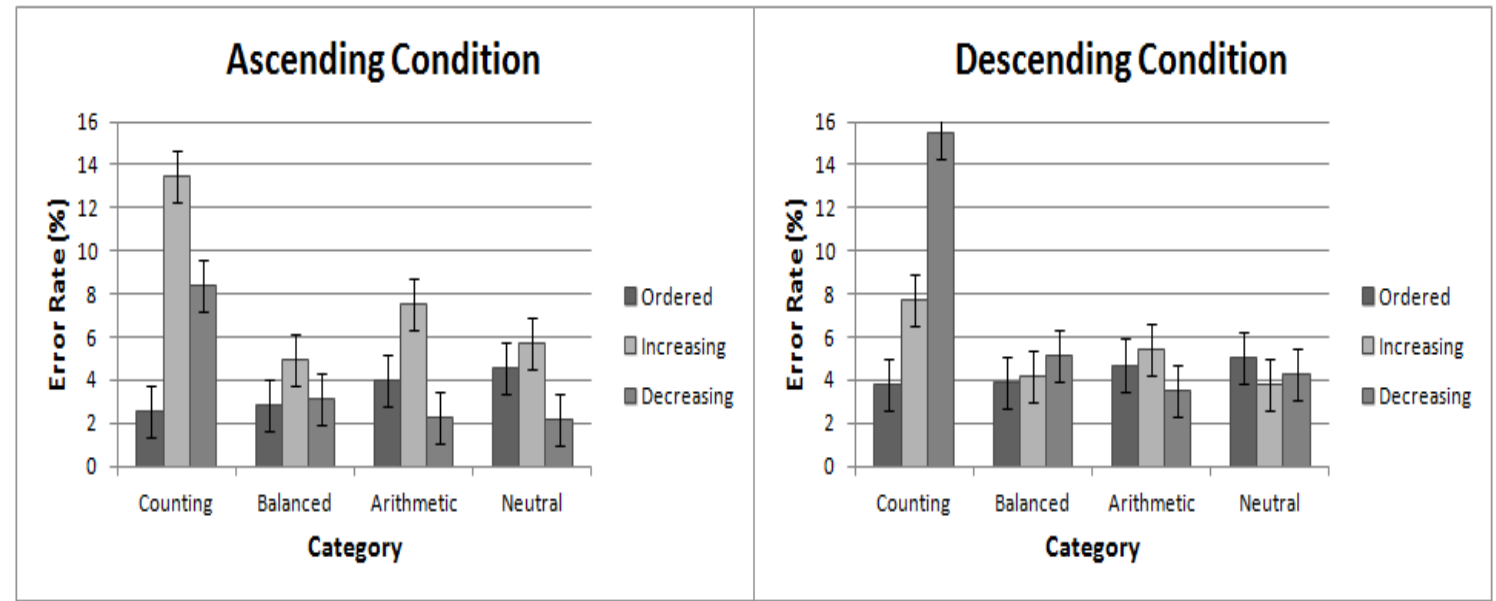

Figure 13. Experiment 2: Ordered, "increasing," and "decreasing” error rates compared across categories and order conditions (Ascending, Descending).

The marginally significant three-way interaction between fluency, sequenceorder, and order condition is shown in Figure 14. Two interesting patterns were found. Comparing across order conditions, low fluency individuals responded more slowly to descending sequences than to ascending sequences, thus supporting the argument that descending sequences are qualitatively different (in terms of familiarity) from ascending. In contrast, high fluency individuals showed similar response times for both ordered types, indicating that this fluency group was either equally familiar with ascending and descending sequences or that they were simply better at recognizing when sequences are ordered. 
The second effect of interest involved the unordered response patterns. For the Ascending condition, response times were quite similar across fluency groups; however, the same was not true for the Descending condition. Comparing across fluency groups, high fluency individuals responded faster to unordered "increasing" sequences in the Descending condition than they did to the same sequences in the Ascending condition (expected, due to these sequences being advantaged by the sequential rejection rule); however, response times for unordered "decreasing" sequences stayed approximately the same instead of getting longer (unexpected, since they were no longer advantaged in the Descending condition). This pattern indicated that high fluency individuals had comparatively less difficulty rejecting unordered "decreasing" sequences, than they did for "increasing" sequences. The fact that only unordered "increasing" sequence response times were substantially longer when not advantaged by a sequential rejection rule indicated that these sequences were inherently more difficult to reject than their “decreasing" counterparts. In contrast, low fluency individuals displayed the expected response pattern reversal, with unordered "increasing" sequences showing shorter latencies in the Descending condition and longer latencies in the Ascending condition, and vice versa for unordered "decreasing" sequences. This pattern suggested that the two unordered types posed comparable levels of difficulty for the low fluency group. 


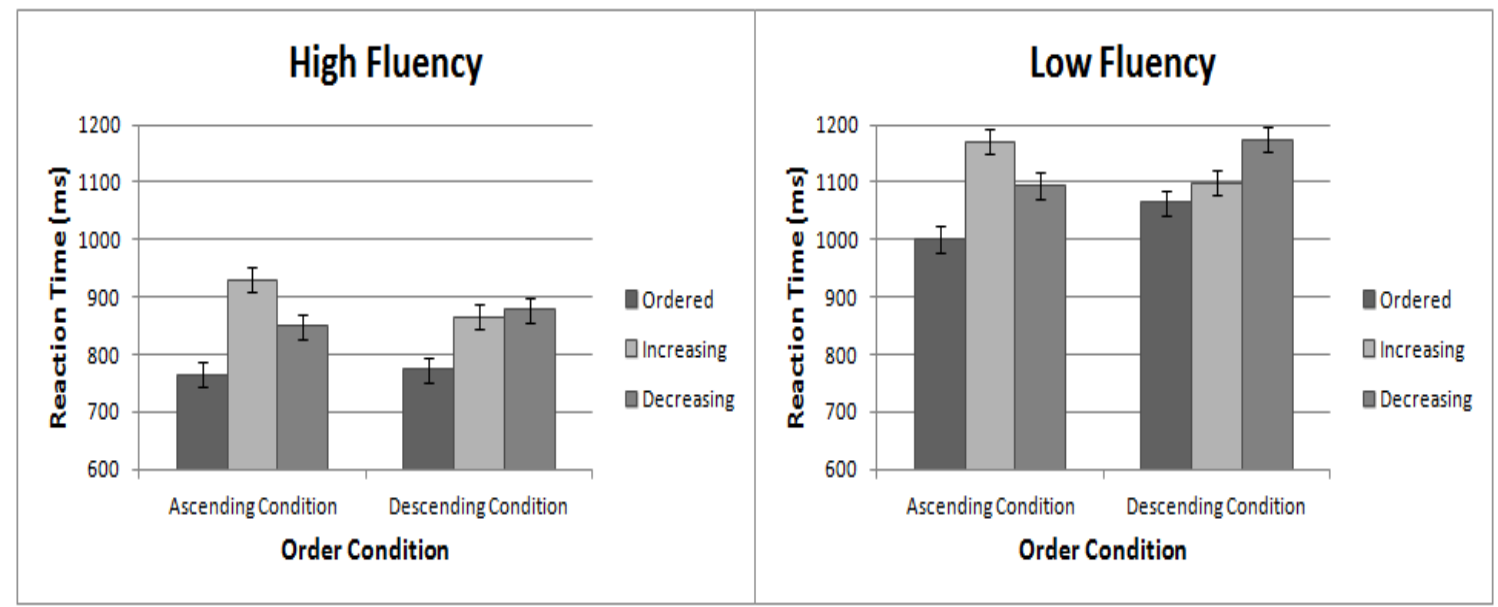

Figure 14. Experiment 2: Ordered, "increasing", and "decreasing" mean response times compared across order conditions (Ascending, Descending) and fluency groups.

In summary, ascending and descending sequences showed fairly comparable response times across all categories in the pure order conditions, except for counting, where ascending sequences showed a definitive speed advantage over descending. When response patterns for these sequence-orders were examined in relation to fluency, low fluency individuals responded more slowly to descending sequences than to ascending, thus providing evidence for the strong influence of familiarity on sequence recognition. In contrast, the high fluency group showed overall similar response times for both ordered types, indicating that these individuals were either more familiar with descending sequences than the low fluency group, or perhaps they were simply better at detecting whether or not sequences were ordered.

For unordered sequences, participants were found to be mainly switching strategies and using reversed versions of the same sequential rejection strategy between the Ascending and Descending order conditions. Essentially, unordered "increasing" sequences were always slower than the ordered and "decreasing" versions in the Ascending order condition. With the exception of arithmetic sequences, unordered 
"decreasing" sequences were always slower than their ordered and "increasing" versions in the Descending condition. When examining fluency-related differences, unordered sequences for low fluency individuals perfectly reflected the implementation of a sequential decision rule. In contrast, high fluency individuals demonstrated an enhanced ability to reject unordered "decreasing" sequences, regardless of order condition; however, "increasing" sequences were relatively more difficult to reject and, unless they were strategically advantaged (as in the Descending condition), showed substantially elevated response times.

Mixed order condition. In the Mixed order condition, both ascending and descending sequences were considered ordered. The median response times for correct trials and percentage of errors were analyzed in separate 2(fluency: low, high) $\mathrm{x}$ 4(category counting, balanced, arithmetic, neutral) x 4(sequence-order: ascending, descending, increasing, decreasing) mixed ANOVAs with sequence-order and category as repeated measures factors and fluency as a between-groups factor (see Table 10 for ANOVA results). All of the main effects and higher-order interactions were significant for both dependent variables. Post hoc testing was also conducted. 
Table 10. ANOVAs (response times and errors) for the Mixed condition

\begin{tabular}{|c|c|c|c|c|c|c|c|}
\hline \multirow[b]{2}{*}{ Source } & \multirow[b]{2}{*}{$d f$} & \multicolumn{3}{|c|}{ Response Time } & \multicolumn{3}{|c|}{ Percent Error } \\
\hline & & $F$ & $p$ & $\eta_{p}^{2}$ & $F$ & $p$ & $\eta_{p}^{2}$ \\
\hline Fluency & 1,76 & $28.15 * *$ & $<.001$ & .270 & $5.94 *$ & .017 & .073 \\
\hline Category & 3,228 & $12.05 * *$ & $<.001$ & .137 & $27.41 * *$ & $<.001$ & .265 \\
\hline Sequence-Order & 2,228 & $54.45 * *$ & $<.001$ & .417 & $8.90 * *$ & $<.001$ & .105 \\
\hline Fluency x Category & 3,228 & $2.44 \dagger$ & .065 & .031 & $5.22 * *$ & .002 & .064 \\
\hline Fluency x Sequence- & 3,228 & $4.35 * *$ & .005 & .054 & $2.67 *$ & .048 & .034 \\
\hline \multicolumn{8}{|l|}{ Order } \\
\hline Category x Sequence- & 9,684 & $29.84 * *$ & $<.001$ & .282 & $16.21 * *$ & $<.001$ & .176 \\
\hline \multicolumn{8}{|l|}{ order } \\
\hline Fluency x Category x & 9,684 & $4.85^{* *}$ & $<.001$ & .060 & $2.27 *$ & .017 & .029 \\
\hline Sequence-Order & & & & & & & \\
\hline
\end{tabular}

As with the previous ANOVAs, high-fluency participants responded faster (958 ms vs. $1346 \mathrm{~ms})$ and made fewer errors (6.4\% vs. 9.2\%) than low-fluency participants. Latencies also varied by category (counting $=1201 \mathrm{~ms}$, balanced $=1143 \mathrm{~ms}$, arithmetic $=$ $1117 \mathrm{~ms}$, neutral $=1147 \mathrm{~ms}$ ), with counting sequences showing longer response latencies and more errors than the other three categories (counting $=10.8 \%$, balanced $=6.0 \%$, arithmetic $=7.3 \%$, neutral $=7.0 \%$ ). Response times were found to also differ by sequence-order $($ ascending $=1016 \mathrm{~ms}$, descending $=1130 \mathrm{~ms}$, "increasing" $=1238 \mathrm{~ms}$, "decreasing" = $1224 \mathrm{~ms}$ ), with ascending sequences showing the fastest response times and fewest errors followed by descending sequences, which were faster than both 
unordered types, but displayed a similarly elevated rate of error (ascending $=5.4 \%$, descending $=8.4 \%$, “increasing" $=8.8 \%$, “decreasing" $=8.5 \%$ ).

A marginally significant two-way interaction was found between category and fluency (see Table 9). Low fluency individuals showed longer response times and more errors for counting sequences (see Figure 15). They also made more errors on arithmetic sequences than on balanced sequences. High fluency individuals likewise made more errors on counting sequences; however, they did not display significantly longer response times for these sequences. This pattern indicated that although counting sequences were challenging for both fluency groups, they posed the most difficulty for low fluency individuals.

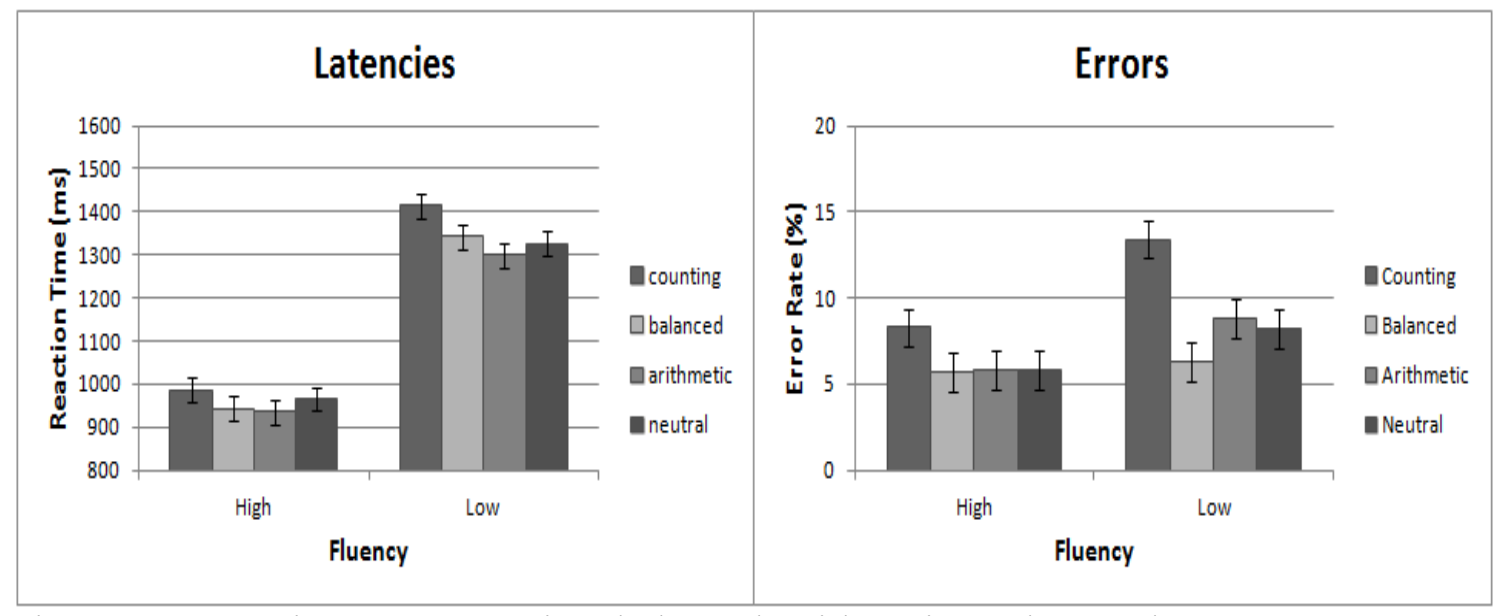

Figure 15. Experiment 2: Counting, balanced, arithmetic, and neutral mean response times and error rates compared across fluency groups in the Mixed condition.

A significant two-way interaction was found between sequence-order and fluency (see Figure 16). Although faster than both unordered types, low fluency individuals responded more slowly to descending sequences than to ascending. Descending sequences were also more similar to unordered with respect to number of errors, whereas ascending sequences showed the fewest errors. This pattern indicated that although being 
ordered (ordinality) does offer a temporal advantage, the greater familiarity of ascending sequences afforded them an even greater overall advantage. In contrast, high fluency individuals showed similar response latencies for the two ordered types. Furthermore, number of errors did not significantly differ between sequence-orders. This pattern suggested that either high fluency individuals have a greater mental store of numerical sequences that includes both ordered types or that they are simply better at detecting when a sequence is ordered.

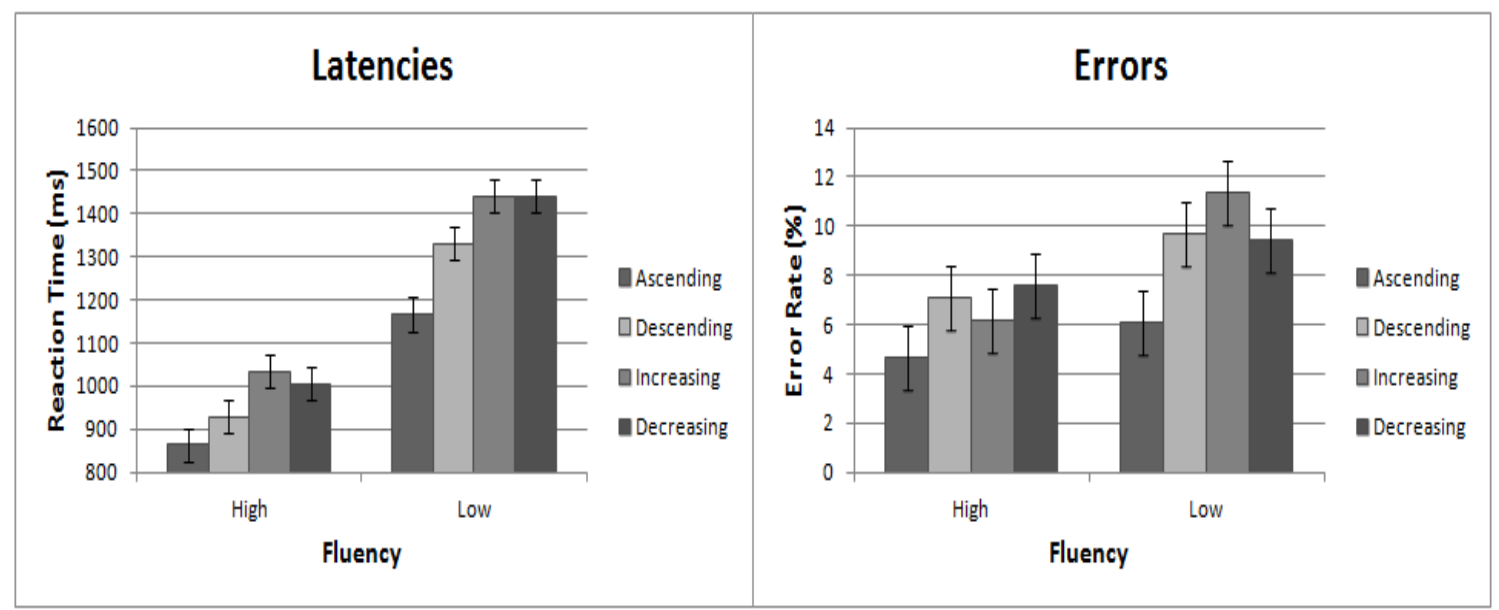

Figure 16. Experiment 2: Ascending, descending, "increasing", and "decreasing" response times and error rates compared across fluency groups.

There was also a significant two-way interaction between category and sequenceorder (see Figure 17), with both unordered counting types (i.e. "increasing" and "decreasing") showing the longest response latencies (unordered "decreasing" sequences were the slowest) and most errors of all four categories. Descending sequences were slower than ascending in all categories, except balanced (which almost met significance). Furthermore, counting and arithmetic descending sequences were both more error prone than their ascending versions. This pattern indicated that, at least in some cases, there was a tendency for less familiar ordered sequences, namely descending, to be mislabelled as unordered. Arithmetic and neutral descending sequences also displayed more errors than 
their unordered "decreasing" counterparts, suggesting that there may have been a bias towards incorrectly classifying these descending sequences as unordered.

Counting ascending sequence had the fastest response latencies, whereas unordered "increasing" sequences had the longest response times of all four sequenceorders in the balanced and arithmetic categories. Arithmetic "increasing" sequences were also more prone to error than their "decreasing" counterparts.

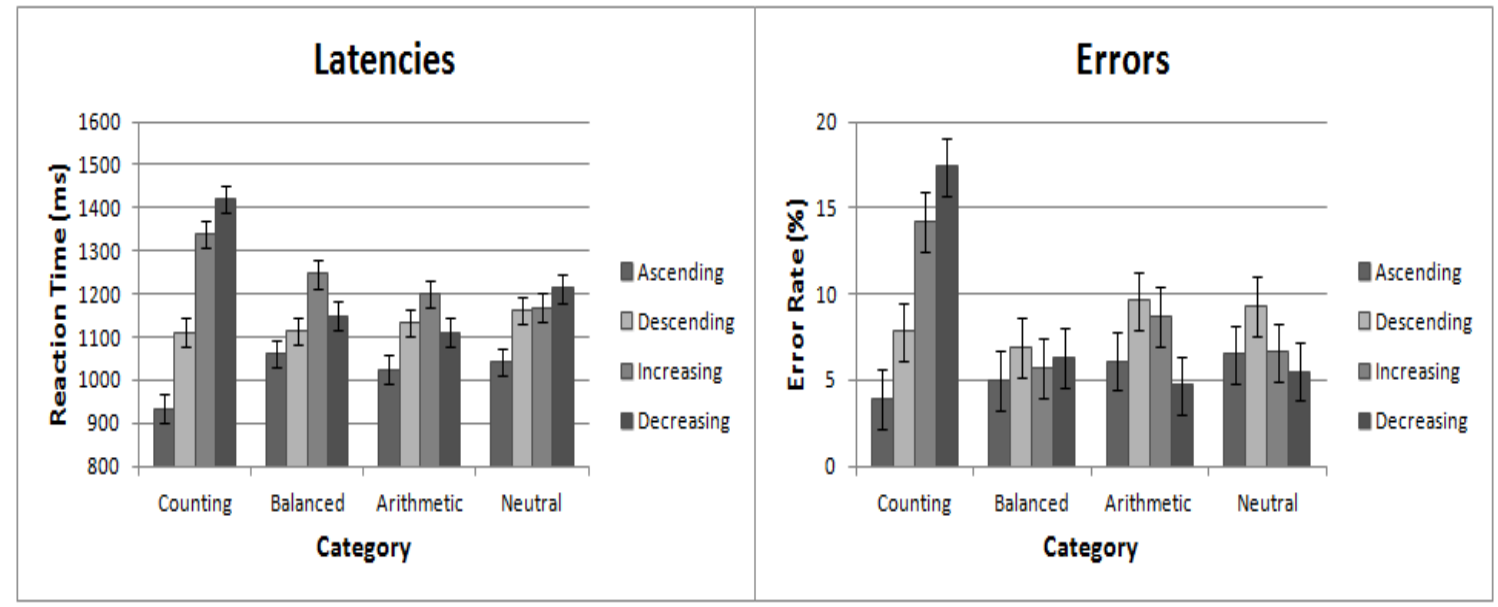

Figure 17. Experiment 2: Ascending, descending, "increasing", and "decreasing" response times and error rates compared across all categories.

A significant three-way interaction was found between category, sequence-order, and fluency (see Figure 18). The response and error patterns for the low fluency groups essentially mimicked those of the previous two-way interaction (sequence-order and category). In each category except balanced (which almost met significance; see Figure 17), low fluency individuals showed the shortest response times for ascending sequences, with counting ascending sequences being the fastest overall and showing fewer errors than their descending counterparts. Unordered counting sequences were again the slowest overall ("decreasing" slower than "increasing") and showed the greatest number of errors. The response patterns for the balanced, arithmetic and neutral categories were, for 
the most part, fairly similar with a few irregularities (e.g. balanced unordered “increasing" sequences were slower than the other balanced sequence-orders). With respect to accuracy, balanced, arithmetic, and neutral sequences were also essentially equivalent, except that arithmetic descending and unordered "increasing" sequences showed more errors than their unordered "decreasing" counterparts.

Although high fluency individuals also responded faster to ascending than to descending counting sequences, the two ordered types had similar latencies across all other categories. Unordered counting sequences were again the slowest overall.

Moreover, unlike the low fluency group, high fluency individuals made more errors on counting unordered "decreasing" sequences, whereas their unordered "increasing" versions showed similar rates of error to the two counting ordered types (see Figure 19). Arithmetic descending sequences were also more error prone than their unordered “decreasing” counterparts.

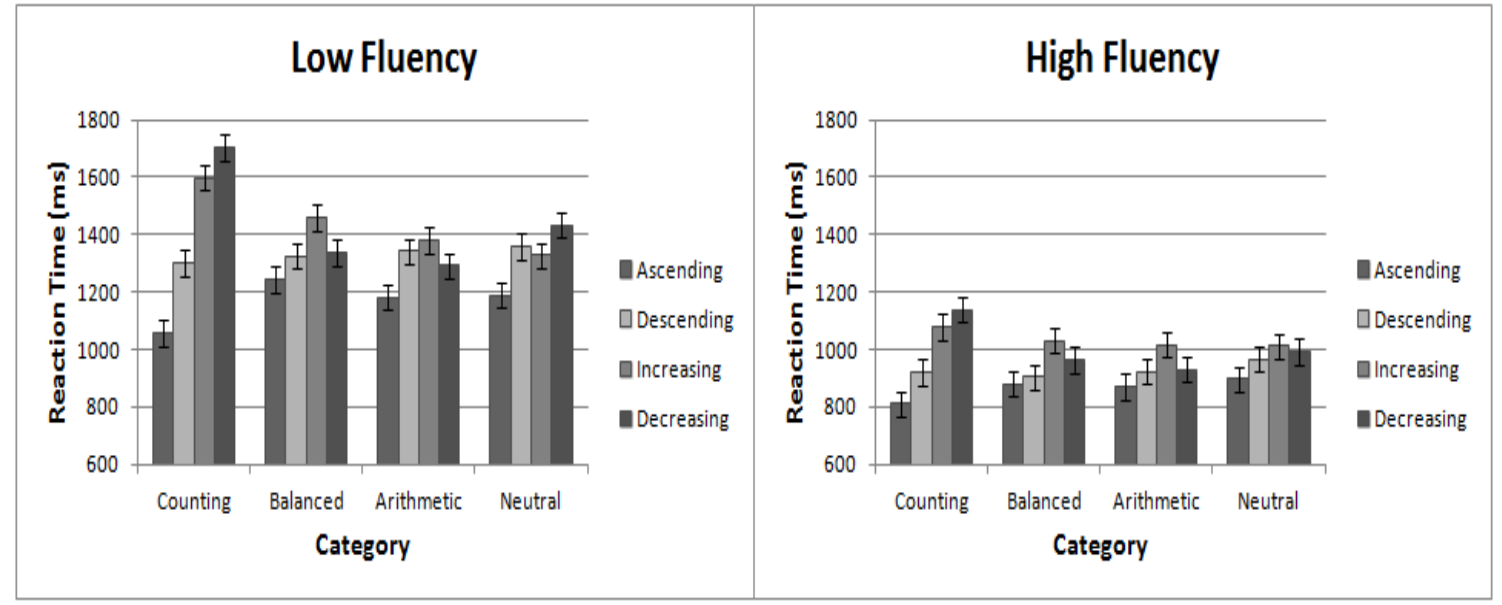

Figure 18. Experiment 2: Ascending, descending, "increasing," and "decreasing" mean response times for both fluency groups across all categories in the Mixed condition. 


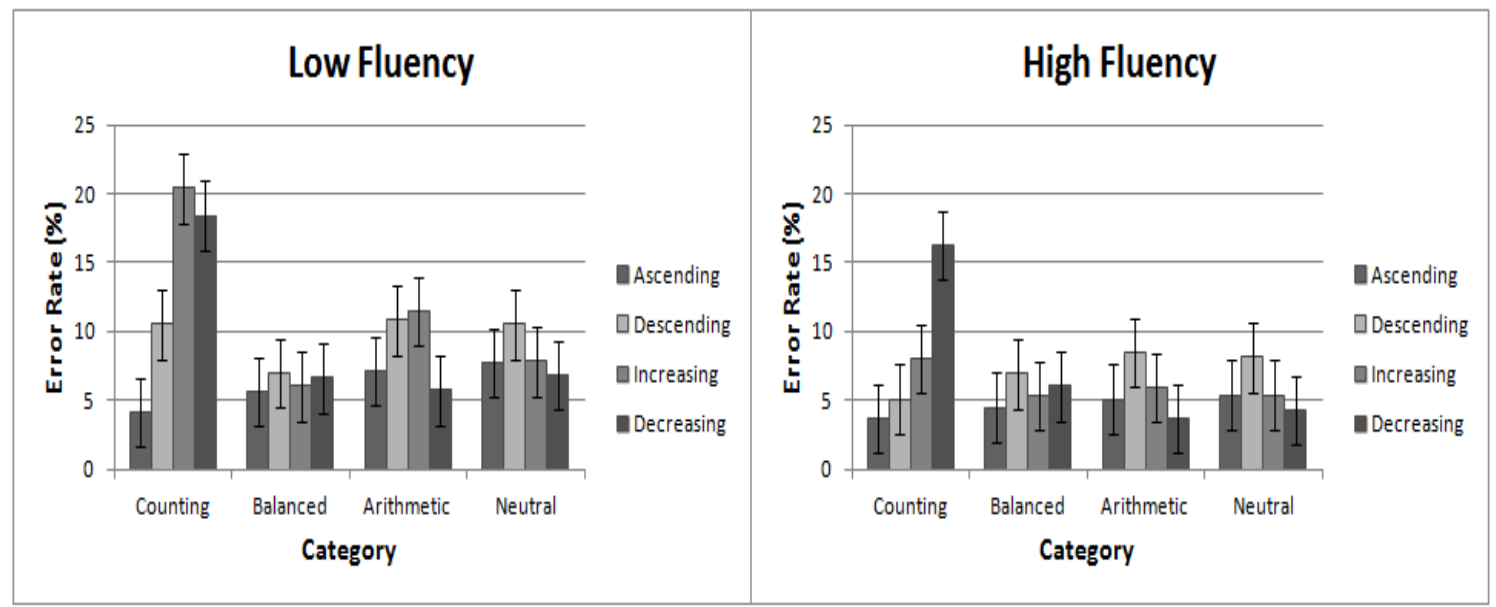

Figure 19. Experiment 2: Ascending, descending, "increasing," and "decreasing" error rates for both fluency groups across all categories in the Mixed condition.

In summary, low-fluency participants were faster and more accurate on ascending sequences than on descending sequences. In contrast, high-fluency participants showed smaller advantages on ascending versus descending sequences than low-fluency participants (e.g., $50 \mathrm{~ms}$ vs. $200 \mathrm{~ms}$ advantage on counting sequences), no latency differences on balanced or arithmetic sequences, and no differences in errors in any category. These findings suggested that high fluency individuals were either more familiar with descending sequences than low fluency individuals or they may be better at perceiving ordinal relationships between numbers; however, even if the latter was true, the fact that counting ascending sequences were faster than descending indicated that strong stimulus familiarity still offered a speed advantage above and beyond what could be accounted for by ordinality alone. However, the presence of strong interference effects demonstrated that familiarity can also have detrimental effects on sequence recognition by making sequence rejection more difficult.

Regarding unordered sequences, the task demands in the Mixed condition prevented participants from using an effective decision rule based on the first two digits (i.e., the sequential decision rule used in the pure order conditions), consequently 
resulting in fairly equivalent "increasing" and "decreasing" response latencies in each category for the high fluency group. However, high fluency individuals still responded more slowly on unordered "increasing" sequences than on ordered sequences in the balanced and arithmetic categories, whereas latencies on unordered "decreasing" response latencies were generally quite similar to those on ordered sequences. This response pattern suggested that high fluency individuals had slightly more difficulty rejecting unordered "increasing" sequences, which was likely due to their very close resemblance to ascending sequences (due to their initial increasing digit pair). For low fluency individuals, arithmetic sequences did not show this pattern and were fairly similar to neutral sequences; however, balanced unordered "increasing” sequences again showed longer latencies than the other sequence-orders. This pattern of responding suggested that, similar to the high fluency group, balanced sequences were relatively more familiar to low fluency individuals than either arithmetic or neutral sequences.

\section{Discussion}

There were two main goals in Experiment 2. The first goal was to further test the role of familiarity versus ordinality as the primary factor in numerical sequence recognition via the inclusion of descending sequences, which are equally ordinal to ascending, but less familiar. In support of the familiarity explanation, ascending sequences were generally responded to more quickly than descending sequences. The second goal was to determine whether participants were using a strategy that involved comparing sequential pairs of digits to determine order. Consistent with this prediction, the unordered "increasing" and "decreasing" sequences showed opposite patterns in the Ascending and Descending order conditions. For example, participants rejected 312 and 
213 more quickly in the Ascending condition, whereas they rejected 231 and 132 more quickly in the Descending condition. This pattern indicated that participants used pairwise relations and processed the stimuli in a left-to-right sequence. The large interference effects for unordered counting sequences were clearly evident, indicating that sequence processing was not quite self-terminating. Instead, these results suggested that there were potentially two different processes occurring: a holistic encoding of all three digits (resulting in activation for unordered counting sequences) followed by, or in parallel with, a sequential, left-to-right decision rule. Both high- and low-fluency participants showed evidence for both of these processing components.

There were some differences as a function of arithmetic fluency. With respect to ordered sequences, low fluency individuals demonstrated an overall speed advantage for ascending sequences over their descending versions. In contrast, the high fluency group only showed a latency advantage on ascending counting sequences, relative to descending counting sequences. Regarding the unordered sequence types, low fluency individuals showed the response pattern reversal between both pure order conditions predicted by the implementation of a sequential decision rule. In contrast, the high fluency group displayed a similar pattern in the Ascending condition, but not for the Descending condition, where equivalent response times were instead observed for the two unordered types. This deviation from what would be expected when using a sequential decision strategy indicated that the high fluency participants had an overall greater difficulty in rejecting unordered "increasing" sequences. This interference was not shared by unordered "decreasing" sequences, suggesting that the main aspect that 
differentiated these two unordered sequence types from each other was the degree to which they resembled ordered ascending sequences.

Finally, there were two sub-goals intended to address some of the limitations associated with Experiment 1. The first involved determining whether or not the interference effect observed for arithmetic sequences had simply been an artefact of having only unordered "increasing" sequences (i.e. no unordered arithmetic "decreasing" sequences were included for comparison), whereas the second sub-goal focused on whether high fluency individuals would show a general interference effect similar to counting sequences (i.e., interference effects for both unordered types) for unordered balanced sequences, particularly in the absence of a sequential decision rule. The subgoals were addressed first, as it impacted whether or not balanced and arithmetic sequences were appropriate comparison groups.

The lack of significant fluency-related differences in the pure order conditions meant that it was necessary to examine responses in the Mixed condition. In the Mixed condition, there was no general interference effect for balanced sequences (i.e., both unordered sequence-orders should have been slower than their ordered versions, but were not); however, small interference effects were observed for unordered "increasing" sequences, with these sequences being significantly slower than both ordered types. Both high and low fluency individuals demonstrated this interference effect for unordered “increasing" sequences. The lack of a similar slowdown for unordered "decreasing" sequences indicated that simply sharing digits with moderately well-known sequences was insufficient to cause major interference; however, in cases where unordered sequences shared digits with moderately familiar sequences and these digits were 
presented in a manner that more closely resembled the familiar ascending sequences (i.e., the first two digits are in ascending order), small interference effects were observed. Therefore, the interference effects observed for balanced sequences were not the same as those observed for counting sequences. Instead, there was simply a general increase in difficulty for high fluency individuals when rejecting unordered sequences that strongly resembled ascending sequences, which were more familiar than descending.

When examining responses from all 78 participants in the pure order conditions, although balanced ascending sequences were faster than their unordered "increasing" counterparts in the Ascending condition, the difference between their ascending and corresponding unordered "decreasing" sequences did not quite reach significance. The Descending condition reflected a similar, but reversed pattern. Essentially, this pattern suggested that for the pure order conditions across both fluency groups, balanced sequences once again did not demonstrate a significant general interference effect.

The second sub-goal focused on evaluating arithmetic sequences for both high and low fluency individuals, and more specifically, determining whether or not the interference effect (i.e., unordered response times $>$ ordered) observed for arithmetic sequences in Experiment 1 did accurately reflect a general interference effect for arithmetic unordered sequences (i.e., both unordered types showing interference) or simply an artefact of having only unordered "increasing" sequences for comparison. The results for Experiment 2 showed that, similar to balanced sequences, there was no general interference effect for arithmetic sequences in the Ascending condition. In contrast, interference effects for both unordered types were observed for arithmetic sequences in the Descending condition. The fact that unordered "increasing" sequences were slower 
than their ordered counterparts, even when advantaged by the sequential rejection strategy, whereas their unordered "decreasing" versions displayed no similar slowdown when receiving the same strategic advantage, indicated that, like balanced sequences, the effect being observed was not a general interference effect. Instead, this small interference effect again indicated that participants had slightly greater difficulty rejecting unordered sequences that both shared digits with and very closely resembled moderately familiar ascending sequences. In the Mixed condition, low fluency individuals did not demonstrate this interference in the form of greater response latencies, but instead made many more errors on unordered "increasing" sequences than on their "decreasing" counterparts. Only high fluency individuals demonstrated a small response time interference effect for arithmetic unordered "increasing" sequences.

To summarize, arithmetic and balanced error and response patterns were by and large quite similar to those of the neutral comparison group. Moreover, the unique effects they did demonstrate were fairly small and highly specific to the order condition, making them of little practical significance. Therefore, neither balanced nor arithmetic categories were examined in further detail or used as comparison groups. Instead, the focus of the discussion regarding the two main objectives for Experiment 2 was made comparing the two familiarity extremes: counting (most familiar) and neutral (least familiar). Neutral sequences acted as the reference group, whereas counting sequences showed very obvious category-related interference effects.

As predicted, the results for Experiment 2 did demonstrate that ordered descending sequences were qualitatively (in terms of familiarity) different from ordered ascending sequences, particularly for low fluency individuals. Examination of participant 
response patterns for the Mixed condition showed that low fluency participants had consistently slower response times for descending sequences than for their ascending versions (see Figure 20). Participants were expected to show similar performance across all four neutral sequence-orders, due to their relative unfamiliarity. Instead, not only were descending neutral sequences slower than ascending sequences, descending sequences displayed response times that were more similar to their unordered versions than did ascending sequences. If the ordinal nature of numerical sequences was the primary factor in their recognition, neutral descending sequences should have, like their ascending counterparts, shown faster response times than their unordered versions. Furthermore, counting and neutral descending sequences should have displayed response times that closely resembled those of their ascending counterparts. As shown in Figure 20, which isolated the counting and neutral sequences from the Mixed condition, patterns for counting and neutral categories varied across sequence-order and fluency.

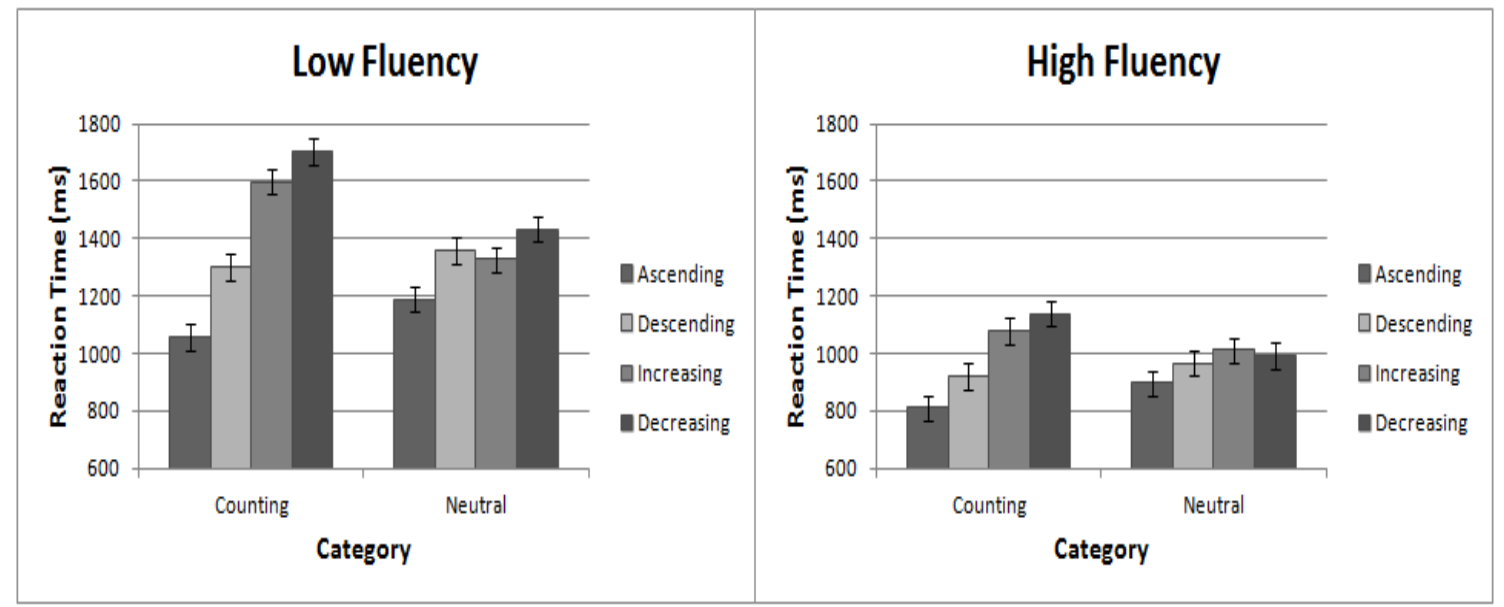

Figure 20. Experiment 2: Ascending, descending, "increasing," and "decreasing" mean response times for the counting and neutral categories compared across fluency groups in the Mixed condition.

The speed advantage that participants showed for ascending sequences over descending sequences indicated that ascending stimuli produced more obligatory 
activation than descending stimuli. This familiarity advantage was particularly

pronounced for ascending counting sequences, which showed the fastest response times of any sequence-order across all experimental conditions and for both fluency groups.

Low-fluency participants (see Figure 21), also made fewer errors on ascending than on descending counting sequences. High-fluency participants, in contrast, only showed disadvantages for errors on unordered "decreasing" counting sequences.

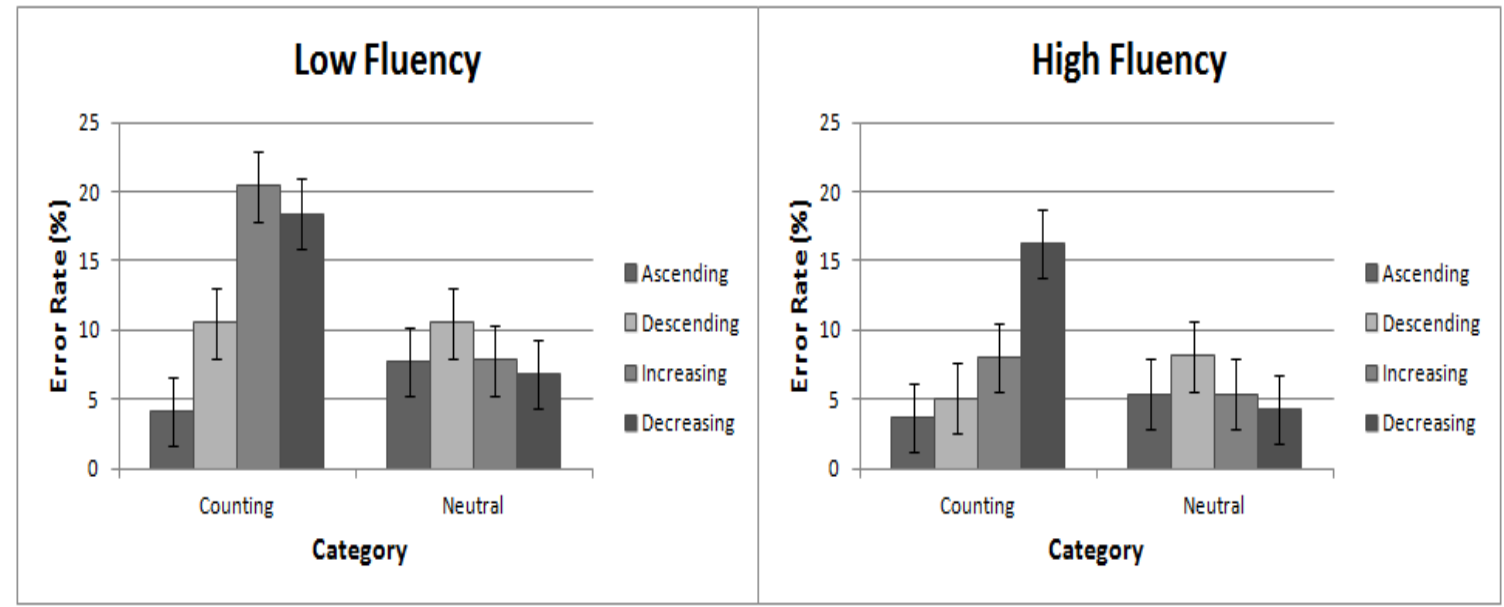

Figure 21. Experiment 2: Ascending, descending, "increasing," and "decreasing" error rates for the counting and neutral categories compared across fluency groups in the Mixed condition.

High fluency individuals also displayed a speed advantage for counting ascending sequences, responding faster to these ascending sequences than to their descending counterparts (see Figure 20). In contrast, the difference in response times between neutral ascending and descending response times did not quite meet significance. These findings could suggest that a) high fluency individuals either are more familiar with descending sequences than low fluency individuals, or b) they may be better at detecting order in general. Although it is difficult to know for certain to which degree $a, b$, or a combination of the two was resulting in the observed equivalencies between the neutral ascending and descending sequences, the fact that descending counting sequences did not display a 
comparable response speed advantage to their ascending counterparts suggested that at least in cases where stimulus familiarity was very high (e.g., ascending counting sequences), high fluency individuals did demonstrate faster and more accurate recognition than could be accounted for by ordinality alone. Furthermore, the fact that in the neutral category (where familiarity was at its lowest), ascending sequences were significantly faster than both unordered types, whereas descending sequences were quite similar to both unordered types, suggested that the familiarity of ascending sequences provided a greater speed advantage than simply being ordered. Moreover, the small interference effects observed for unordered "increasing" sequences in the balanced and arithmetic categories, which could not be explained by the use of a sequential rejection rule, indicated that even for the high fluency group, rejecting sequences that closely resembled ordered ascending sequences was often more difficult than rejecting unordered sequences that did not.

The second main objective was to determine if individuals were using a sequential rejection strategy that exploited contextual factors, such as the experimental design (i.e., knowing an experimental condition had only a specific type of ordered sequence; ascending sequences in the Ascending condition and descending sequences in the Descending condition), to evaluate unordered sequences. I predicted that participants would be slower and less accurate on unordered "increasing" sequences than on “decreasing" sequences in the Ascending condition (essentially replicating Experiment 1), whereas they would be faster and show fewer errors than unordered "decreasing" sequences in the Descending condition. As predicted, participant response patterns did show condition-specific advantages for unordered sequences. Unordered "decreasing" 
sequences were faster and easier to reject than their "increasing" versions in the Ascending condition, whereas an almost identical, but reversed pattern was found for the Descending condition, with unordered "increasing" sequences being more quickly evaluated and rejected (see Figure 22). The cross-over latency pattern was identical for counting and neutral sequences (differences of 50-60 ms in all four instances), suggesting that the process which produced these effects was the same regardless of sequence category. In contrast, it is very clear from Figure 22 that differences between ordered and unordered sequences were much larger for counting than for neutral sequences, and larger for ascending than descending sequences. These effects were consistent with the view that the familiarity of the counting sequences had independent effects on processing.

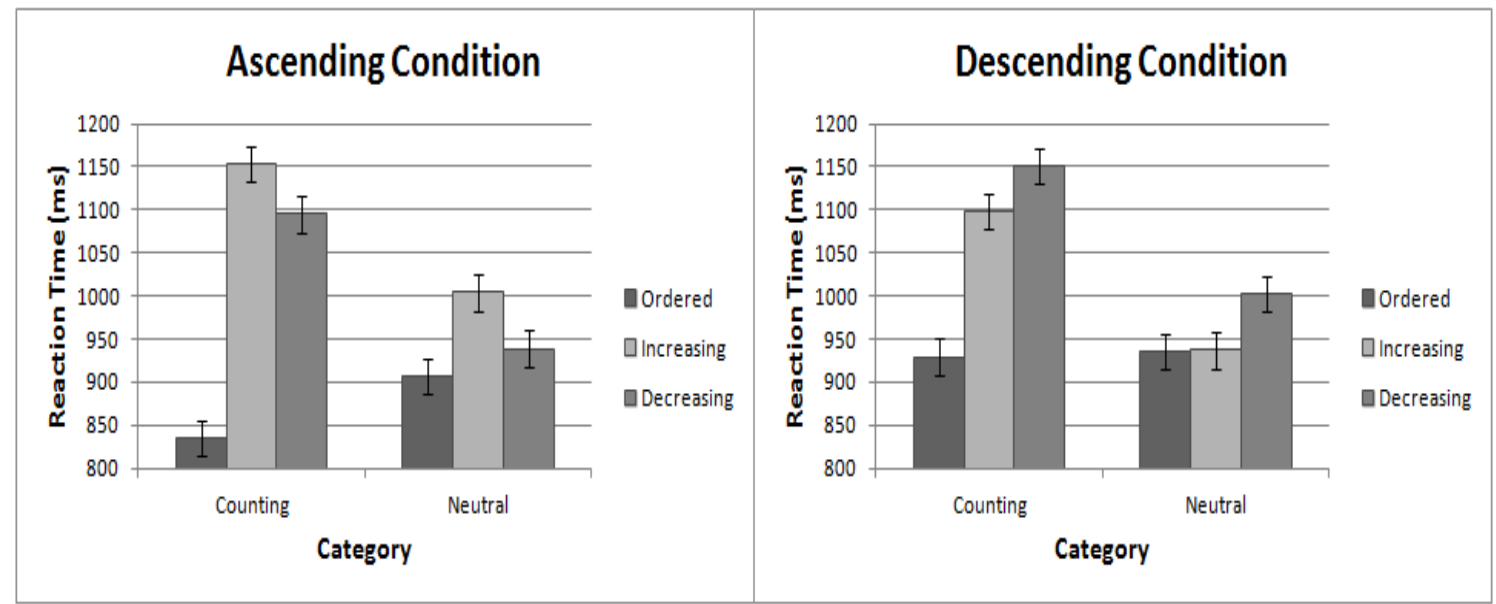

Figure 22. Experiment 2: Ordered, "increasing," and "decreasing" mean response times for the counting and neutral categories in the pure order conditions (Ascending, Descending).

When examined in relation to fluency, the response pattern remained essentially the same as previously described for the low fluency group; however, high fluency individuals had shown some important differences. Although the highly skilled group similarly demonstrated response times for unordered "increasing" sequences that were 
slower than their "decreasing" counterparts in the Ascending condition and "increasing" counterparts in the Descending condition (thus indicating the implementation of a sequential decision rule), unordered “decreasing" sequences showed fairly similar response times across order conditions and were essentially equivalent to their unordered “increasing" versions in the Descending condition. This pattern of results indicated that high fluency individuals have more difficulty rejecting unordered "increasing" sequences and that this difficulty likely arises from the fact that of the two unordered types, unordered "increasing" sequences more closely resembled ordered ascending sequences (i.e., until the third digit an unordered "increasing" sequence is identical to an ordered ascending sequence). Therefore, the longer response times found for unordered “increasing” sequences were evidence of small interference effects, where high fluency individuals had relatively more difficulty rejecting these unordered sequences that so closely resembled ordered ascending sequences. 


\section{GENERAL DISCUSSION}

The objective of the current research was to examine whether familiarity or ordinality was the primary factor in numerical sequence recognition and to determine how these factors were influenced by arithmetic fluency. The study comprises two experiments, both involving the completion of an ordering task and an arithmetic fluency measure. Comparisons were made between four categories of numerical sequences (Counting, Balanced, Arithmetic, Neutral) and four sequence-orders (ascending, descending, "increasing”, “decreasing"). Due to limitations in the first experiment, the second experiment served as both a more complete replication, as well as extension, of the first.

The primary results of the current research were as follows. As expected, highly-skilled participants typically responded much faster than their less-skilled counterparts. Ordered ascending sequences were also responded to more quickly than descending sequences, supporting the familiarity explanation of performance. As predicted, participants implemented a strategy that used a sequential decision rule to facilitate the rejection of unordered sequences that exploited the experimental design (i.e. Ascending condition vs. Descending condition. Both fluency groups exploited this strategic advantage, however high fluency individuals appeared to only need help from this sequential decision rule when dealing with unordered "increasing" sequences in the Descending condition. Furthermore, the high fluency group continued to show small interference effects for unordered "increasing" sequences in the balanced and arithmetic categories in the Mixed condition, indicating that highly skilled individuals have significantly more automatic (obligatory) activation, when facing highly familiar numerical sequences (i.e., despite being unordered, "increasing" sequences were very 
similar to ordered ascending sequences). Regarding general interference effects, the highly familiar counting sequences showed very large interference effects for both unordered types. Balanced and arithmetic sequences showed no general effects for either fluency group and thus were excluded as comparison groups from further study.

\section{Familiarity vs. Ordinality}

In both experiments, counting ordered sequences (i.e. ascending) displayed the fastest response times and fewest errors overall for both fluency groups. Recall that Lyons and Beilock $(2009 ; 2011)$ have suggested that of the different category-types, counting ordered sequences possessed the greatest degree of ordinal association between their constituent digits, due to their small and regular inter-digit intervals. Moreover, an individual's ability to perceive and process this ordinal information is what permits them to make quick and accurate order judgements. With ordered counting sequences being the most "ordinal," both high and low skilled individuals should be able to quickly recognize and respond to these sequences, whereas ordered neutral sequences, which are far less ordinal due to their larger and irregular intervals, should display slower response times that are more similar to their unordered sequences. This reasoning seems unlikely for several reasons.

Firstly, in terms of order relationships among digits, counting sequences (e.g., 45 6), are just as ordered as neutral sequences (e.g., 13 7). Ordinal or ranking data is only informative insofar that it indicates what number comes before or after another. There is no information regarding the size of the intervals between the numbers themselves, and even if that information was available, it does not affect how ordered a sequence is. Therefore, in terms of "orderedness," the different ordered sequences (i.e. counting, 
neutral, etc.) are all equally in order. Secondly, in both the current study and Lyons and Beilock (2009), participants responded to unordered counting sequences more slowly and less accurately than to unordered sequences in the other categories. If a greater degree of ordinal association between digits was the primary factor affecting recognition, then all unordered sequences across the various categories should display similarly slow response times, whereas ordered sequences should become increasingly faster as the degree of ordinal association between digits supposedly increases. However, this was not the case. The large number of errors and very slow response times for unordered counting sequences pointed to substantial interference effects resulting from the obligatory activation of mentally stored, or familiar, numerical information that must be inhibited, resulting in increased task difficulty. Notably, interference effects were much larger for low-skilled individuals than for high-skilled, which indicated that the high fluency group was much more efficient at inhibiting affirmative responses to the automatic activation or false recognition.

Thirdly, ordered descending sequences were included in Experiment 2 of the current study. If the degree of ordinal association was the primary determinant of speedy and accurate sequence recognition, then ascending and descending sequences should show equivalent response times and rates of error. Instead, for ordered counting sequences, descending sequences were solved more slowly and less accurately than their ascending counterparts. The same was found for neutral sequences. Low fluency individuals responded much more slowly to descending than to ascending neutral sequences. In fact, descending neutral sequences were treated more like unordered sequences, which could not be explained by ordinality. Although the difference between 
ascending and descending response times was not significant for high fluency

individuals, response latencies for descending sequences strongly resembled their unordered versions, whereas ascending response times were significantly faster than both unordered types. These response patterns indicated that individuals are more accustomed to seeing and recognizing ascending numerical sequences. Simply being in order (e.g., descending sequences), is not sufficient for speedy recognition. Therefore, familiarity with certain groupings of numbers (e.g., 34 2), as well as observing them in a wellknown and meaningful pattern (e.g., 23 4), yields the most obligatory activation of related numerical information, which can result in the speedy and accurate recognition of familiar number sequences (e.g., ordered sequences) or strong interference effects for sequences that closely resemble these familiar sequences (e.g., unordered sequences).

\section{Strategy Implementation}

Examination of the unordered sequences in Experiment 1 revealed a distinctive pattern for unordered sequences, with "increasing" sequences being slower than their “decreasing" counterparts. To determine if this distinctive and consistent pattern resulted from unordered "increasing” sequences being inherently more difficult, or as a byproduct of the experimental design, three order conditions were introduced in Experiment 2 (i.e., Ascending, Descending, and Mixed). As expected, participants were using a sequential decision strategy in the pure order conditions (i.e., Ascending, Descending) that advantaged one unordered type over the other, by checking whether or not the initial digit pair conformed to the type of ordered sequence expected in that specific order condition. This advantage allowed both fluency groups to more quickly and easily reject the 
unordered sequences that did not conform to the expected pattern of the type of ordered sequence contained within the order condition.

Notably, participants showed substantial interference for unordered counting sequences independently of the small advantage for some unordered sequences, indicating that the third digit was always at least partially processed. The sequential decision process was likely always occurring either in parallel with or after initial processing of the complete sequence.

\section{Limitations and Future Research}

One limitation of the current study was that it was not possible to determine if other strategies were also being used and whether these might be influenced by participants' skill. Therefore, the addition of participant strategy questionnaires and eyetracking in future research studies on numerical sequence recognition could be highly informative. Strategy questionnaires would permit some investigation into the conscious awareness that participants have over their strategy selection, whereas eye-tracking could allow detailed observations of exactly how participants visually tackle different kind of numerical sequences and potentially reveal additional strategies.

Another direction for future research involves in-depth analysis of arithmetic and balanced sequences. Although the interference effects found for these categories were quite small, more specific analyses could be made regarding particular items within the categories themselves. For example, balanced sequences such as 246,468 , or 369 , would likely be considered quite familiar as they comprise part of the multiplication tables for the numbers 2 and 3. Other balanced sequences, such as 159 or 147 , do not. Therefore, although not as familiar as a counting sequence, item analyses of particular 
sub-groupings of balanced sequences could potentially reveal interference effects similar to those of counting sequences, or at least larger versions of the effects observed in the current study. Regarding arithmetic sequences, the lack of corresponding increases in response time and number of errors for unordered "decreasing" sequences in the Descending condition was unexpected. The factors contributing to the ease of rejection for this type of unordered sequence are currently unknown and additional investigation may be required.

\section{CONCLUSIONS}

Based on the results of the current study, familiarity provides a better explanation for the patterns found by Lyons and Beilock $(2009,2011)$ than ordinality. Unlike ordinality, stimulus familiarity is able to account for all of the findings of the current research, including (a) interference effects, (b) faster responding and fewer errors on ordered ascending sequences in comparison to descending, and (c) the advantage of ordered counting sequences over ordered neutral sequences (i.e., they are all equally ordered, but counting ordered sequences are more familiar than are neutral sequences). Furthermore, the fact that high-fluency individuals demonstrated a greater overall processing speed, inhibitory control, and mental store of meaningful numerical information (such as numerical sequences), can help account for why these individuals have a greater advantage over less-skilled individuals with respect to arithmetic performance. The advantages exhibited by highly-skilled individuals enable them to more quickly and effectively recognize numerical sequences than less-skilled individuals, while inhibiting non-relevant information, which is invaluable for both basic math learning and more complex arithmetic. 
Regarding strategy use, both high- and low-fluency individuals used readily available strategies to increase task efficiency and reduce task load. In the current study, individuals were able to use the experimental design to formulate a sequential decision rule for the pure order conditions that strongly impacted participant response patterns. The results indicated that two processes occurred in this task, obligatory activation of associations along with a sequential decision rule. Individual differences in performance may be more strongly related to the first than the second process, although more research is needed to understand exactly how sequence processing and arithmetic skill are related. 


\section{REFERENCES}

Agrillo, C., Piffer, L., \& Bisazza, A. (2010). Large number discrimination by mosquitofish. PloS one, 5(12), e15232.

Anderson, U. S., \& Cordes, S. (2013). $1<2$ and 2<3: non-linguistic appreciations of numerical order. Frontiers in psychology, 4.

Andersson, G., Hagman, J., Talianzadeh, R., Svedberg, A., \& Larsen, H. C. (2002). Effect of cognitive load on postural control. Brain research bulletin, 58(1), 135139.

Ashcraft, M. H. (1982). The development of mental arithmetic: A chronometric approach. Developmental Review, 2(3), 213-236.

Ashcraft, M. H. (1983). Simulating Network Retrieval of Arithmetic Facts.

Ashcraft, M. H. (1987). Children's knowledge of simple arithmetic: A developmental model and simulation. In C.J. Brainerd, R. Kail, \& J. Bisanz (Eds.), Formal methods of developmental research (pp. 302-338). New York: Springer-Verlag.

Ashcraft, M. H. (1992). Cognitive arithmetic: A review of data and theory. Cognition, 44(1), 75-106.

Baroody, A. J. (1984). A reexamination of mental arithmetic models and data: A reply to Ashcraft. Developmental Review, 4(2), 148-156.

Bisazza, A., Piffer, L., Serena, G., \& Agrillo, C. (2010). Ontogeny of numerical abilities in fish. Plos One, 5(11), e15516.

Booth, J. L., \& Siegler, R. S. (2008). Numerical magnitude representations influence arithmetic learning. Child Development, 79(4), 1016-1031. doi:10.1111/j.14678624.2008.01173.x 
Brannon, E. M. (2002). The development of ordinal numerical knowledge in infancy. Cognition, 83(3), 223-240.

Brannon, E. M., Cantlon, J. F., \& Terrace, H. S. (2006). The role of reference points in ordinal numerical comparisons by rhesus macaques (macaca mulatta). Journal of Experimental Psychology: Animal Behavior Processes, 32(2), 120.

Brannon, E. M., \& Terrace, H. S. (1998). Ordering of the numerosities 1 to 9 by monkeys. Science, 282(5389), 746-749.

Brannon, E. M., \& Terrace, H. S. (2000). Representation of the numerosities 1-9 by rhesus macaques (Macaca mulatta). Journal of Experimental Psychology: Animal Behavior Processes, 26(1), 31.

Butterworth, B., Zorzi, M., Girelli, L., \& Jonckheere, A. R. (2001). Storage and retrieval of addition facts: The role of number comparison. The Quarterly Journal of Experimental Psychology: Section A, 54(4), 1005-1029.

Campbell, J. I., \& Oliphant, M. (1992). Representation and retrieval of arithmetic facts: A network-interference model and simulation.

Campbell, J. I. (1996). Mechanisms of simple addition and multiplication: A modified network-interference theory and simulation. Mathematical Cognition: Vol. 1, 1, $121-164$.

Campbell, J. I. D., \& Xue, Q. (2001). Cognitive arithmetic across cultures. Journal of Experimental Psychology: General, 130(2), 299-315. doi:10.1037/00963445.130.2.299

Cohen Kadosh, R., Dowker, A., Heine, A., Kaufmann, L., \& Kucian, K. (2013). Interventions for improving numerical abilities: Present and future. Trends in 
Neuroscience and Education.

Colegatef, R. L., Hoffman, J. E., \& Eriksen, C. W. (1973). Selective encoding from multielement visual displays. Perception \& Psychophysics, 14(2), 217-224.

Collins, A. M., \& Loftus, E. F. (1975). A spreading-activation theory of semantic processing. Psychological review, 82(6), 407.

Cordes, S., \& Brannon, E. M. (2008). The difficulties of representing continuous extent in infancy: Using number is just easier. Child development, 79(2), 476-489.

D'Amico, A., \& Passolunghi, M. C. (2009). Naming speed and effortful and automatic inhibition in children with arithmetic learning disabilities. Learning and Individual Differences, 19(2), 170-180.

Dehaene, S. (1997). The number sense: How the mind creates mathematics. Oxford University Press.

DeStefano, D., \& LeFevre, J. A. (2004). The role of working memory in mental arithmetic. European Journal of Cognitive Psychology, 16(3), 353-386.

Emmerton, J. (1998). Numerosity differences and effects of stimulus density on pigeons' discrimination performance. Animal Learning \& Behavior, 26(3), 243-256.

Eriksen, C. W., \& Collins, J. F. (1969). Temporal course of selective attention. Journal of experimental psychology, 80(2p1), 254.

Eriksen, C. W., \& Hoffman, J. E. (1972). Temporal and spatial characteristics of selective encoding from visual displays. Perception \& psychophysics, 12(2), 201-204.

Eriksen, C. W., \& Rohrbaugh, J. W. (1970). Some factors determining efficiency of selective attention. The American Journal of Psychology, 330-342.

Forster, K. I., \& Hector, J. (2002). Cascaded versus noncascaded models of lexical and 
semantic processing: The turple effect. Memory \& Cognition, 30(7), 1106-1117.

Geary, D. C. (1993). Mathematical disabilities: cognitive, neuropsychological, and genetic components. Psychological bulletin, 114(2), 345.

Geary, D. C., Hoard, M. K., \& Bailey, D. H. (2012). Fact retrieval deficits in low achieving children and children with mathematical learning disability. Journal of learning disabilities, 45(4), 291-307.

Hasher, L., Zacks, R. T., \& May, C. P. (1999). Inhibitory control, circadian arousal, and age.

Hecht, S. A. (1999). Individual solution processes while solving addition and multiplication math facts in adults. Memory \& Cognition, 27(6), 1097-1107.

Hecht, S. A. (2002). Counting on working memory in simple arithmetic when counting is used for problem solving. Memory \& Cognition, 30, 447-455.

Holloway, I. D., \& Ansari, D. (2009). Mapping numerical magnitudes onto symbols: The numerical distance effect and individual differences in children's mathematics achievement. Journal of experimental child psychology, 103(1), 17-29.

Imbo, I., \& Vandierendonck, A. (2008). Practice effects on strategy selection and strategy efficiency in simple mental arithmetic. Psychological Research/Psychologische Forschung, 72(5), 528-541. doi:10.1007/s00426-007-0128-0

Imbo, I., Vandierendonck, A., \& De Rammelaere, S. (2007). The role of working memory in the carry operation of mental arithmetic: Number and value of the carry. The Quarterly Journal of Experimental Psychology, 60(5), 708-731. doi:10.1080/17470210600762447

Jacob, S. N., \& Nieder, A. (2008). The ABC of cardinal and ordinal number 
representations. Trends in cognitive sciences, 12(2), 41-43.

Jordan, K. E., \& Brannon, E. M. (2006). Weber's Law influences numerical representations in rhesus macaques (Macaca mulatta). Animal Cognition, $9(3), 159-172$.

Krueger, L. E. (1986). Why $2 \times 2=5$ looks so wrong: On the odd-even rule in product verification. Memory \& Cognition, 14(2), 141-149.

LeFevre, J.-A., Bisanz, J., Daley, K. E., Buffone, L., Greenham, S. L., \& Sadesky, G. S. (1996). Multiple routes to solution of single digit multiplication problems. Journal of Experimental Psychology:General, 125, 284-306.

LeFevre, J.-A., \& Bisanz, J. (1986). A cognitive analysis of number-series problems Sources of individual-differences in performance. Memory \& Cognition, 14, 287298.

LeFevre, J. A., Bisanz, J., Daley, K. E., Buffone, L., Greenham, S. L., \& Sadesky, G. S. (1996). Multiple routes to solution of single-digit multiplication problems. Journal of Experimental Psychology: General, 125(3), 284.

Lefevre, J. A., Bisanz, J., \& Mrkonjic, L. (1988). Cognitive arithmetic: Evidence for obligatory activation of arithmetic facts. Memory \& Cognition, 16(1), 45-53.

LeFevre, J.-A., Sadesky, G. S., \& Bisanz, J. (1996). Selection of procedures in mental addition: Reassessing the problem size effect in adults. Journal of Experimental Psychology: Learning, Memory, \& Cognition, 22, 216-230

LeFevre, J., DeStefano, D., Coleman, B., \& Shanahan, T. (2005). Mathematical cognition and working memory. Handbook of mathematical cognition, 361-378.

Lemaire, P., \& Fayol, M. (1995). When plausibility judgments supersede fact retrieval: 
The example of the odd-even effect on product verification. Memory \& Cognition, 23(1), 34-48.

Lewis, B. P., \& Linder, D. E. (1997). Thinking about choking? Attentional processes and paradoxical performance. Personality and Social Psychology Bulletin, 23(9), 937944.

Lipton, J. S., \& Spelke, E. S. (2003). Origins of Number Sense Large-Number Discrimination in Human Infants. Psychological Science, 14(5), 396-401.

Lipton, J. S., \& Spelke, E. S. (2004). Discrimination of large and small numerosities by human infants. Infancy, 5(3), 271-290.

Lochy, A., Seron, X., Delazer, M., \& Butterworth, B. (2000). The odd-even effect in multiplication: Parity rule or familiarity with even numbers?. Memory \& cognition, 28(3), 358-365.

Lyons, I. M., \& Beilock, S. L. (2009). Beyond quantity: individual differences in working memory and the ordinal understanding of numerical symbols. Cognition, 113(2), 189-204. Elsevier B.V. doi:10.1016/j.cognition.2009.08.003

Lyons I. M., Beilock S. L. (2011). Numerical ordering ability mediates the relation between number-sense and arithmetic competence. Cognition 121 256-261.

MacLeod, C. M., Dodd, M. D., Sheard, E. D., Wilson, D. E., \& Bibi, U. (2003). In opposition to inhibition. Psychology of learning and motivation, 43, 163-214.

Masson, M. E., \& Loftus, G. R. (2003). Using confidence intervals for graphically based data interpretation. Canadian Journal of Experimental Psychology/Revue canadienne de psychologie expérimentale, 57(3), 203.

Mulhern, G., \& Wylie, J. (2004). Changing levels of numeracy and other core 
mathematical skills among psychology undergraduates between 1992 and 2002. British Journal of Psychology, 95(3), 355-370. doi:10.1348/0007126041528176

Nairne, J. S., \& Healy, A. F. (1983). Counting backwards produces systematic errors.

Journal of Experimental Psychology: General, 112(1), 37.

Neely, J. H. (1976). Semantic priming and retrieval from lexical memory: Evidence for facilitatory and inhibitory processes. Memory \& Cognition, 4(5), 648-654.

Neely, J. H. (1977). Semantic priming and retrieval from lexical memory: Roles of inhibitionless spreading activation and limited-capacity attention. Journal of Experimental Psychology: General, 106(3), 226.

Passolunghi, M. C., \& Siegel, L. S. (2001). Short-term memory, working memory, and inhibitory control in children with difficulties in arithmetic problem solving. Journal of Experimental Child Psychology, 80(1), 44-57.

Prather, R. W., \& Alibali, M. W. (2009). The development of arithmetic principle knowledge: How do we know what learners know?. Developmental Review, 29(4), 221-248.

Simoneau, M., Teasdale, N., Bourdin, C., Bard, C., Fleury, M., \& Nougier, V. (1999). Aging and postural control: postural perturbations caused by changing the visual anchor. Journal of the American Geriatrics Society, 47(2), 235.

Sweller, J. (1988). Cognitive load during problem solving: Effects on learning. Cognitive science, 12(2), 257-285.

Tzelgov, J., \& Ganor-Stern, D. (2005). Automaticity in processing ordinal information. Handbook of mathematical cognition, 55-67. 
Verguts, T., \& Fias, W. (2005). Interacting neighbors: A connectionist model of retrieval in single-digit multiplication. Memory \& cognition, 33(1), 1-16.

Wood, J. N., \& Spelke, E. S. (2005). Infants' enumeration of actions: Numerical discrimination and its signature limits. Developmental Science, 8(2), 173-181.

Xu, F., \& Spelke, E. S. (2000). Large number discrimination in 6-month-old infants. Cognition, 74(1), B1-B11.

Zamarian, L., Ischebeck, A., \& Delazer, M. (2009). Neuroscience of learning arithmetic_Evidence from brain imaging studies. Neuroscience and Biobehavioral Reviews, 33(6), 909-925. doi:10.1016/j.neubiorev.2009.03.005

Van Zandt, T., \& Townsend, J. T. (1993). Self-terminating versus exhaustive processes in rapid visual and memory search: An evaluative review. Perception \& Psychophysics, 53(5), 563-580. 


\section{Appendix A}

Table A.1. Complete list of all experimental stimuli used in Experiment 1.

\begin{tabular}{|c|c|c|c|c|c|}
\hline & Ascending & 1-Unordered & 2-Unordered & 3-Unordered & 4-Unordered \\
\hline \multirow[t]{7}{*}{ Counting } & 123 & 132 & & 231 & \\
\hline & 234 & & 324 & & 423 \\
\hline & 345 & 354 & & & 534 \\
\hline & 456 & 465 & & & 645 \\
\hline & 567 & 576 & 657 & & \\
\hline & 678 & 687 & & & 867 \\
\hline & 789 & 798 & & & 978 \\
\hline \multirow[t]{9}{*}{ Balanced } & 135 & 153 & 315 & & \\
\hline & 147 & & 417 & 471 & \\
\hline & 159 & 195 & 519 & & \\
\hline & 246 & & & 462 & 624 \\
\hline & 258 & 285 & & & \\
\hline & 357 & 375 & & & 735 \\
\hline & 369 & 396 & & & 936 \\
\hline & 468 & 486 & & & 846 \\
\hline & 579 & 597 & 759 & & \\
\hline \multirow[t]{12}{*}{ Arithmetic } & 134 & 143 & & & \\
\hline & 145 & & & 451 & \\
\hline & 156 & 165 & & & \\
\hline & 167 & 176 & & & \\
\hline & 178 & 187 & & & \\
\hline & 189 & 198 & & & \\
\hline & 257 & & & 572 & \\
\hline & 268 & 286 & & & \\
\hline & 279 & 297 & & & \\
\hline & 347 & 374 & & & \\
\hline & 358 & 385 & & & \\
\hline & 459 & 495 & & & \\
\hline \multirow[t]{10}{*}{ Neutral } & 124 & & & 241 & \\
\hline & 125 & & 215 & & \\
\hline & 126 & & & 261 & \\
\hline & 127 & 172 & & & \\
\hline & 128 & 182 & & & \\
\hline & 129 & & 219 & & \\
\hline & 138 & 183 & & & \\
\hline & 139 & 193 & & & \\
\hline & 146 & & 416 & & \\
\hline & 148 & & & & 814 \\
\hline
\end{tabular}




\begin{tabular}{|c|c|c|c|c|}
\hline 149 & & & 491 & \\
\hline 157 & & & & 715 \\
\hline 158 & & & 581 & \\
\hline 168 & & 618 & & \\
\hline 179 & 197 & & & \\
\hline 237 & & & 372 & \\
\hline 238 & & & & 823 \\
\hline 239 & & 329 & & \\
\hline 249 & & 429 & & \\
\hline 259 & 295 & & & \\
\hline 269 & & & & 926 \\
\hline 278 & & & & 827 \\
\hline 289 & & & & 892 \\
\hline 346 & & 436 & & \\
\hline 348 & 384 & & & 934 \\
\hline 349 & & & & \\
\hline 356 & & & 563 & \\
\hline 367 & & & 673 & \\
\hline 368 & & 638 & & \\
\hline 359 & 395 & & & \\
\hline 379 & 397 & & & \\
\hline 457 & & 547 & & 745 \\
\hline 458 & & 548 & & \\
\hline 467 & 476 & & & \\
\hline 469 & & & 694 & \\
\hline 478 & & & & 847 \\
\hline 479 & 497 & & & \\
\hline 489 & & & 894 & \\
\hline 569 & & & & 956 \\
\hline 589 & 598 & & & \\
\hline 679 & & 769 & & \\
\hline 689 & & & & 968 \\
\hline
\end{tabular}


Table A.2. Complete list of all stimuli used in Experiment 2.

\begin{tabular}{|c|c|c|c|c|c|c|}
\hline & Ascending & Descending & Unordered & Unordered & Unordered & Unordered \\
\hline \multirow[t]{7}{*}{ Counting } & 123 & 321 & 132 & 213 & 231 & 312 \\
\hline & 234 & 432 & 243 & 324 & 342 & 423 \\
\hline & 345 & 543 & 354 & 435 & 453 & 534 \\
\hline & 456 & 654 & 465 & 546 & 564 & 645 \\
\hline & 567 & 765 & 576 & 657 & 675 & 756 \\
\hline & 678 & 876 & 687 & 768 & 786 & 867 \\
\hline & 789 & 987 & 798 & 879 & 897 & 978 \\
\hline \multirow[t]{9}{*}{ Balanced } & 135 & 531 & 153 & 315 & 351 & 513 \\
\hline & 147 & 741 & 174 & 417 & 471 & 714 \\
\hline & 159 & 951 & 195 & 519 & 591 & 915 \\
\hline & 246 & 642 & 264 & 426 & 462 & 624 \\
\hline & 258 & 852 & 285 & 528 & 582 & 825 \\
\hline & 357 & 753 & 375 & 537 & 573 & 735 \\
\hline & 369 & 963 & 396 & 639 & 693 & 936 \\
\hline & 468 & 864 & 486 & 648 & 684 & 846 \\
\hline & 579 & 975 & 597 & 759 & 795 & 957 \\
\hline \multirow[t]{12}{*}{ Arithmetic } & 134 & 431 & 143 & 341 & 314 & 413 \\
\hline & 145 & 541 & 154 & 451 & 415 & 514 \\
\hline & 156 & 651 & 165 & 561 & 516 & 615 \\
\hline & 167 & 761 & 176 & 671 & 617 & 716 \\
\hline & 178 & 871 & 187 & 781 & 718 & 817 \\
\hline & 189 & 981 & 198 & 891 & 819 & 918 \\
\hline & 257 & 752 & 275 & 572 & 527 & 725 \\
\hline & 268 & 862 & 286 & 682 & 682 & 826 \\
\hline & 279 & 972 & 297 & 792 & 729 & 927 \\
\hline & 347 & 743 & 374 & 473 & 437 & 734 \\
\hline & 358 & 853 & 385 & 583 & 538 & 835 \\
\hline & 459 & 954 & 495 & 594 & 549 & 945 \\
\hline \multirow[t]{12}{*}{ Neutral } & 124 & 421 & 142 & 214 & 241 & 412 \\
\hline & 126 & 621 & 162 & 216 & 261 & 612 \\
\hline & 136 & 631 & 163 & 316 & 361 & 613 \\
\hline & 138 & 831 & 183 & 318 & 381 & 813 \\
\hline & 149 & 941 & 194 & 419 & 491 & 914 \\
\hline & 237 & 732 & 273 & 327 & 372 & 723 \\
\hline & 239 & 932 & 293 & 329 & 392 & 923 \\
\hline & 269 & 962 & 296 & 629 & 692 & 926 \\
\hline & 348 & 843 & 384 & 438 & 483 & 834 \\
\hline & 378 & 873 & 387 & 738 & 783 & 837 \\
\hline & 457 & 754 & 475 & 547 & 574 & 745 \\
\hline & 478 & 874 & 487 & 748 & 784 & 847 \\
\hline
\end{tabular}


489

984

498

849

894

948

589

598

859

895

958 
Appendix B 
ID

\section{$\underline{\text { Practice }}$}

This is paper and pencil task designed to determine how quickly and accurately you can do addition, subtraction and multiplication. There will be a page of each kind of problem.

Before starting the task, please do the practice problems below to familiarize you with the types of problems that you will see. For each type of practice problem, the first item has been worked out for you.

- Please write your answers in the boxes below the problems.

o You may use the extra space on the page for rough work.

$\circ$ Try to be quick and accurate.

\section{Addition Practice Problems:}
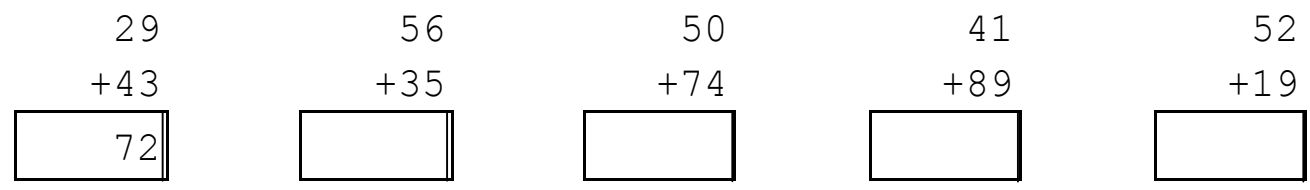

$\underline{\text { Subtraction Practice Problems: }}$
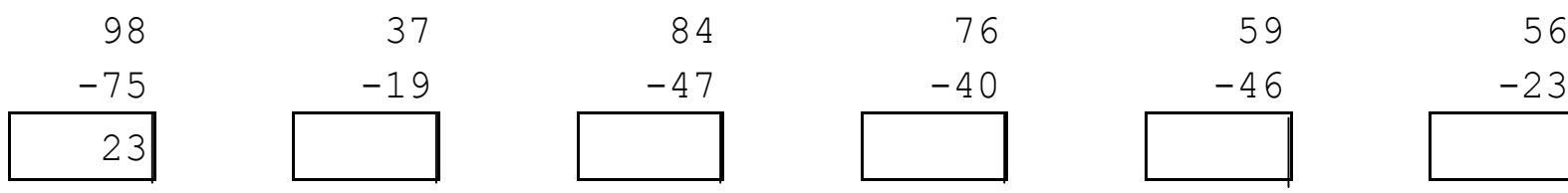

Multiplication Practice Problems:
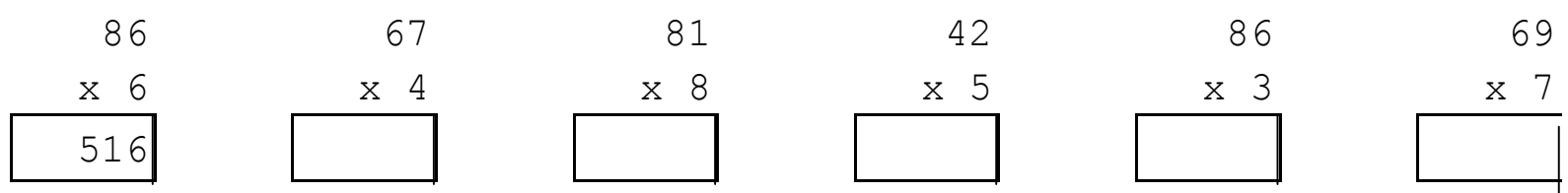

You will have 1 minute for each of the three parts of this test. Each part has one page. You are not expected to finish all of the problems in the time allowed. Your score on this test will be the number of problems done correctly. You will work on the problems from left to right, and then move down to the next row. Do not skip any problems. Work as rapidly as you can without sacrificing accuracy.

If you do finish a part, STOP. Please do not go on to the next part until you are asked to do so. 
Addition

Part 1 (1 minute)
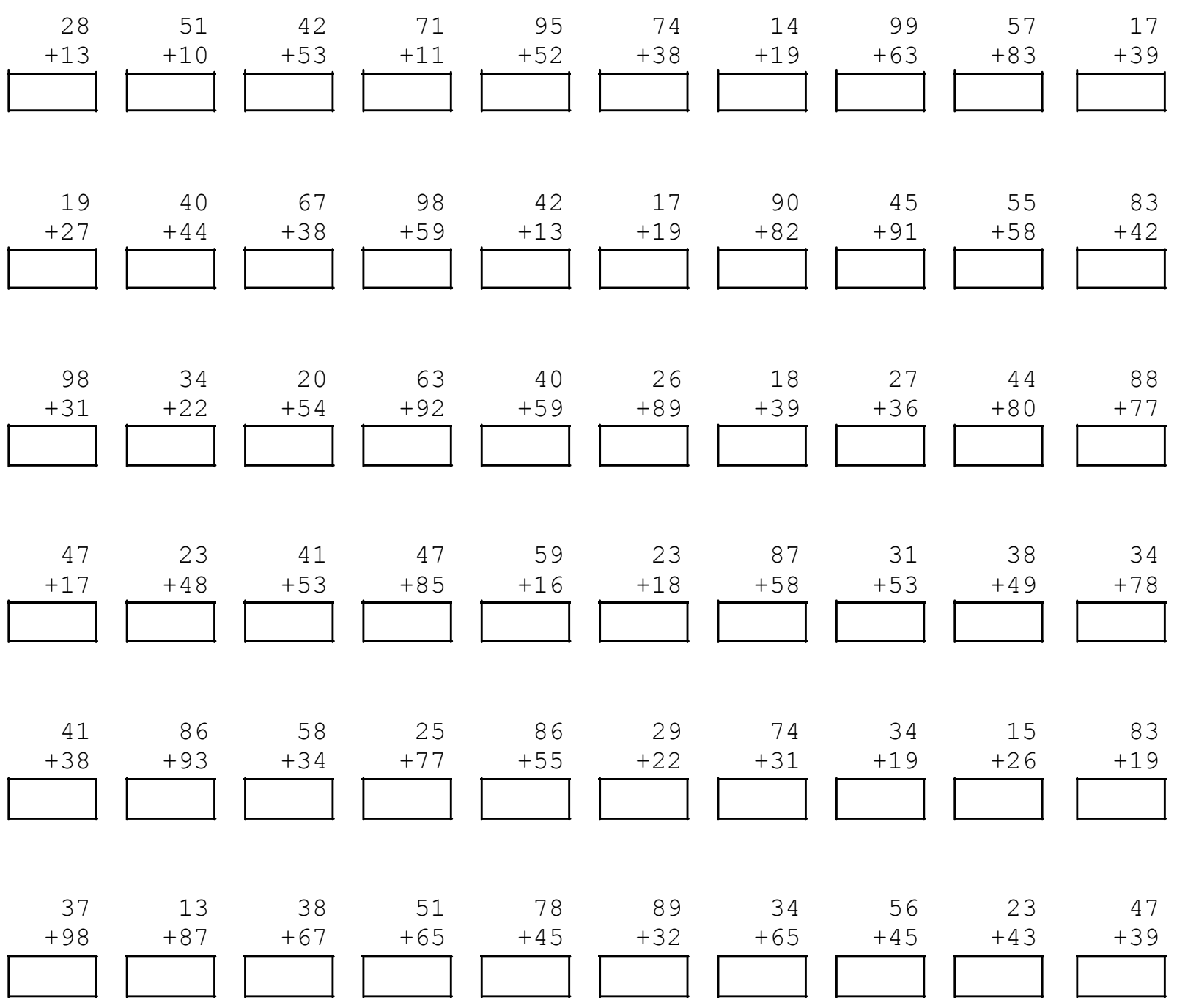

DO NOT TURN THIS PAGE UNTIL ASKED TO DO SO. 
Subtraction

Part 2 (1 minute)
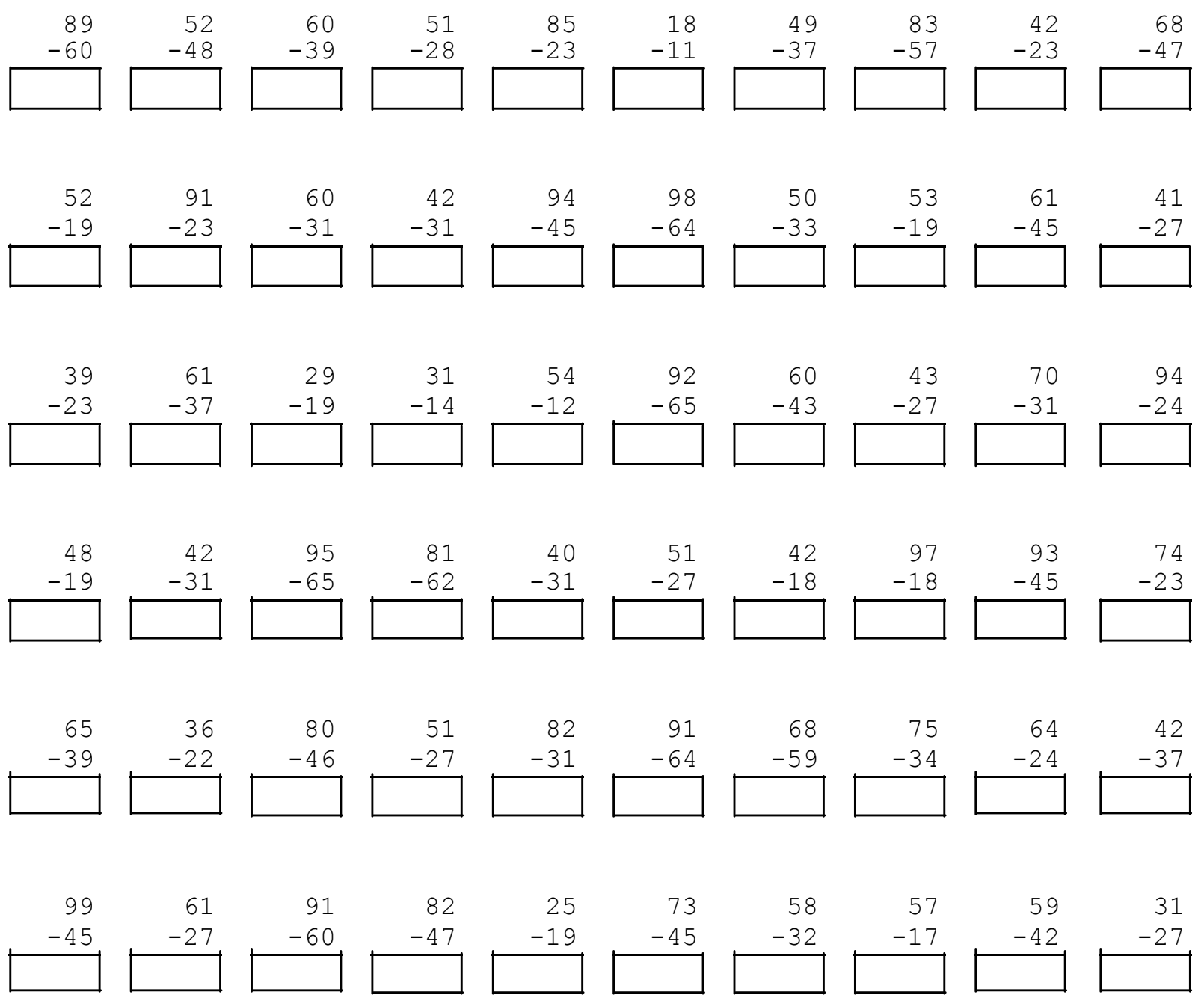

DO NOT GO BACK AND

DO NOT TURN THE PAGE UNTIL ASKED TO DO SO. 
Multiplication

Part 3 (1 minute)

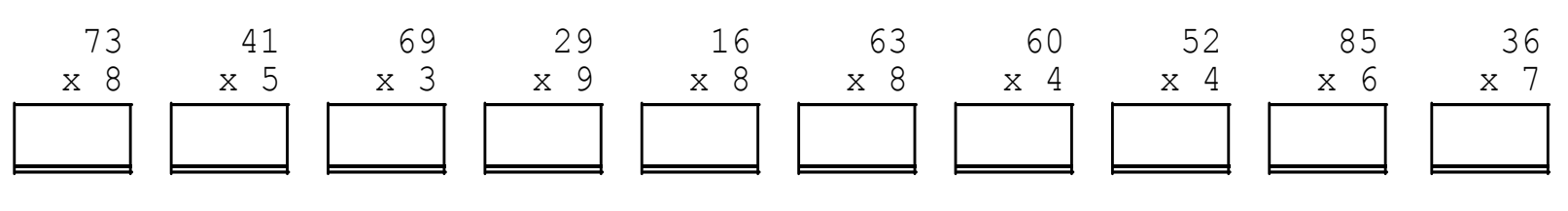

\begin{tabular}{rrrrrrrrr}
52 & 98 \\
$\times 9$ & $\times 3$ \\
\hline
\end{tabular}

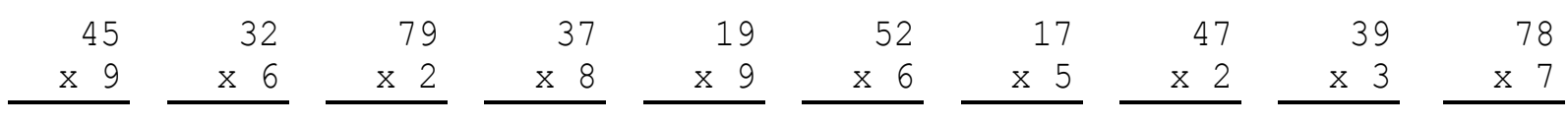

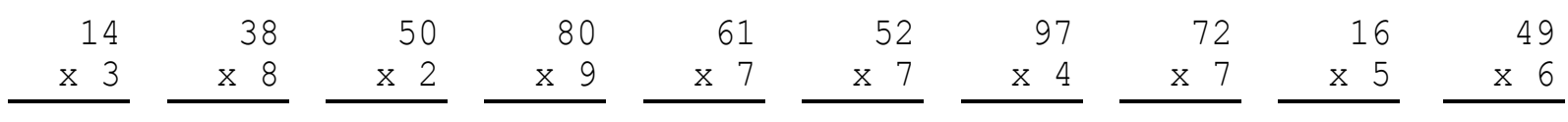

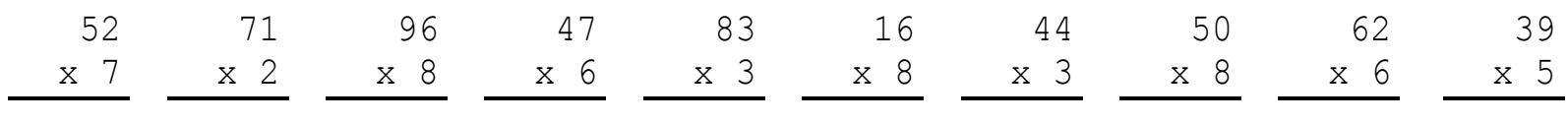

\begin{tabular}{rrrrrrrrrr}
13 & 68 \\
$\times \quad 8$ & $\times 4$ \\
\hline \hline
\end{tabular}

DO NOT GO BACK TO ANY OTHER PAGE

Figure 23. Fluency measure used to calculate participants' arithmetic fluency scores. 